\title{
CATALAN ADDENDUM
}

\author{
Richard P. Stanley
}

version of 25 May 2013

The problems below are a continuation of those appearing in Chapter 6 of Enumerative Combinatorics, volume 2. Combinatorial interpretations of Catalan numbers are numbered as a continuation of Exercise 6.19, while algebraic interpretations are numbered as a continuation of Exercise 6.25. Combinatorial interpretations of Motzkin and Schröder numbers are numbered as a continuations of Exercise 6.38 and 6.39, respectively. The remaining problems are numbered 6.C1, 6.C2, etc. I am grateful to Emeric Deutsch for providing parts (ooo), $\left(\mathrm{a}^{4}\right),\left(\mathrm{f}^{4}\right),\left(\mathrm{z}^{4}\right),\left(\mathrm{g}^{5}\right),\left(\mathrm{p}^{5}\right),\left(\mathrm{d}^{6}\right),\left(\mathrm{u}^{7}\right),\left(\mathrm{v}^{7}\right),\left(\mathrm{x}^{7}\right)$ and $\left(\mathrm{y}^{7}\right)$ of Exercise 6.19, and to Roland Bacher for providing $\left(\mathrm{g}^{6}\right)$.

Note. In citing results from this Addendum it would be best not to use the problem numbers (or at the least give the version date), since I plan to insert new problems in logical rather than numerical order.

Note. At the end of this addendum is a list of all problems added on or after December 17, 2001, together with the date the problem was added. The problem numbers always refer to the version of the addendum in which the list appears.

Note. Throughout this addendum we let $C_{n}$ denote the Catalan number $\frac{1}{n+1}\left(\begin{array}{c}2 n \\ n\end{array}\right)$ and $C(x)$ the generating function

$$
C(x)=\sum_{n \geq 0} C_{n} x^{n}=\frac{1-\sqrt{1-4 x}}{2 x} .
$$

Moreover, we let

$$
\begin{aligned}
& E(x)=\frac{C(x)+C(-x)}{2}=\sum_{n \geq 0} C_{2 n} x^{2 n} \\
& O(x)=\frac{C(x)-C(-x)}{2}=\sum_{n \geq 0} C_{2 n+1} x^{2 n+1} .
\end{aligned}
$$


6.19(ooo) Plane trees with $n-1$ internal nodes, each having degree 1 or 2 , such that nodes of degree 1 occur only on the rightmost path

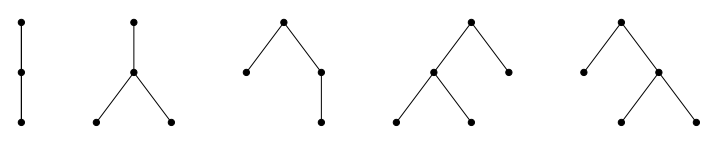

(ppp) Plane trees with $n$ vertices, such that going from left to right all subtrees of the root first have an even number of vertices and then an odd number of vertices, with those subtrees with an odd number of vertices colored either red or blue

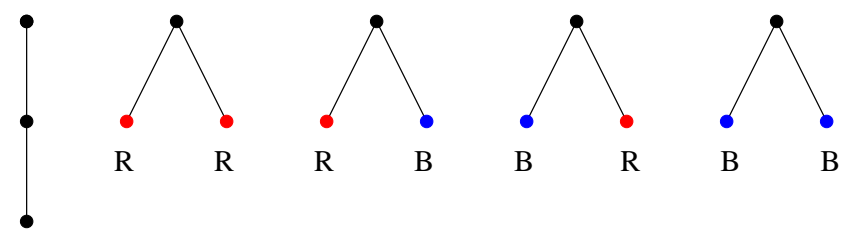

(qqq) Plane trees with $n$ vertices whose leaves at height one are colored red or blue
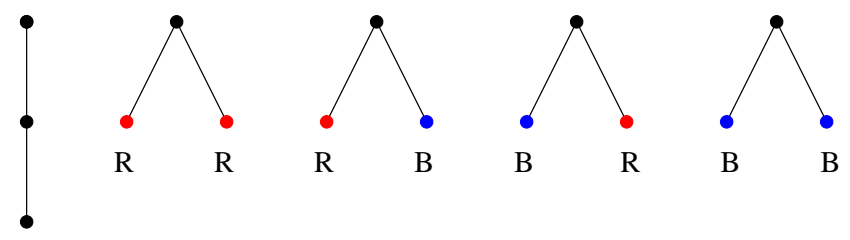

(rrr) Plane trees with $n$ internal vertices such that each vertex has at most two children and each left child of a vertex with two children is an internal vertex

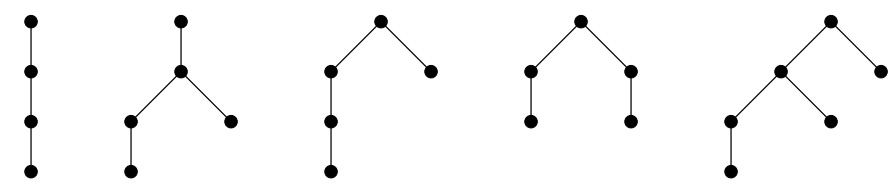

(sss) Plane trees for which every vertex has 0,1 , or 3 children, with a total of $n+1$ vertices with 0 or 1 child 


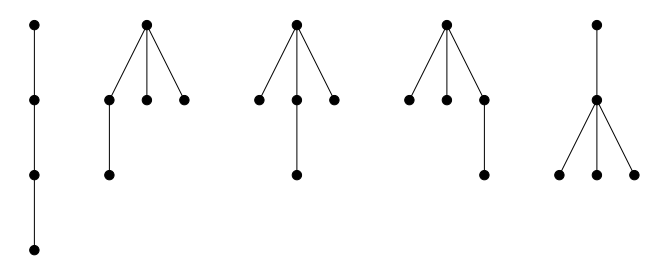

(ttt) Bicolored (i.e., each vertex is colored red or blue with no monochromatic edge) unrooted plane trees (i.e., unrooted trees with the subtrees at each vertex cyclically ordered) with $n+1$ vertices, rooted at an edge (marked $*$ below)
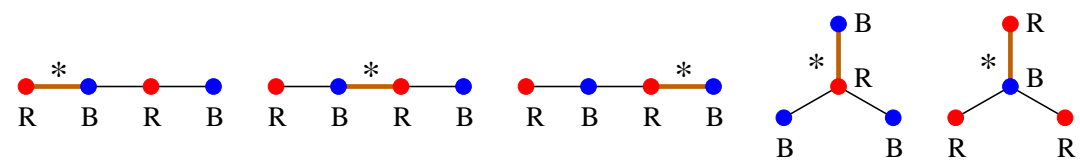

(uuu) Plane trees with $n+1$ vertices, each nonroot vertex labelled by a positive integer, such that (i) leaves have label 1, (ii) any nonroot, nonleaf vertex has label no greater than the sum of its children's labels, and (iii) the only edges with no right neighbor are those on the rightmost path from the root
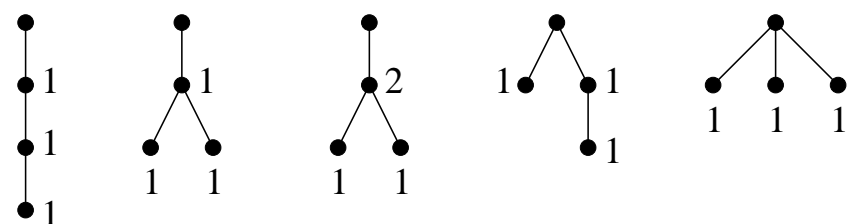

(vvv) Increasing plane trees on the vertex set $[n+1]$ with increasing leaves in preorder (or from left-to-right), such that the path from the root 1 to $n+1$ contains every nonleaf vertex
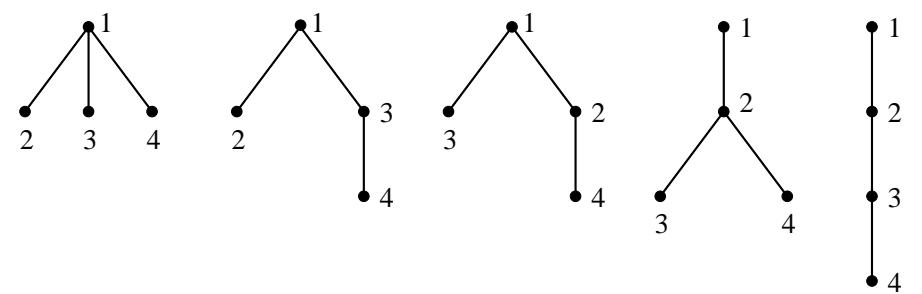

(www) Rooted trees with $n$ vertices such that each nonleaf vertex has either a left child, a middle child, a right child, or a left and right 
child, and such that every left child has a left child, a right child, or both
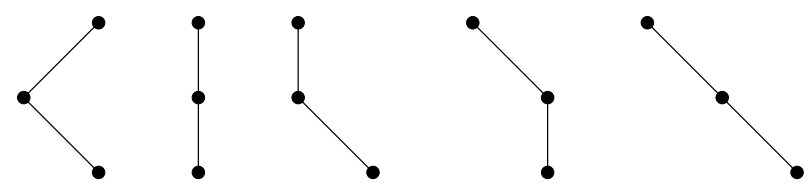

(xxx) Induced subtrees with $n$ edges, rooted at an endpoint, of the hexagonal lattice, up to translation and rotation<smiles>CCCCC</smiles><smiles>CCCC</smiles><smiles>CCCC</smiles><smiles>CCCC</smiles><smiles>CC(C)C</smiles>

(yyy) Noncrossing increasing trees on the vertex set $[n+1]$, i.e., trees whose vertices are arranged in increasing order around a circle such that no edges cross in their interior, and such that all paths from vertex 1 are increasing
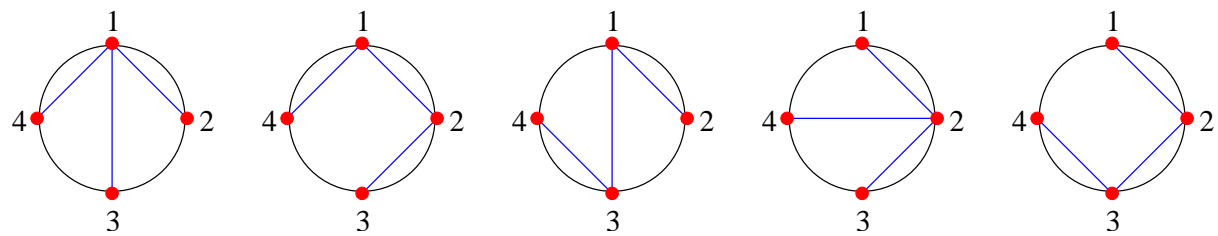

(zzz) Nonnesting increasing trees on the vertex set $[n+1]$, i.e., trees with root 1 such that there do not not exist vertices $h<i<j<k$ such that both $h k$ and $i j$ are edges, and such that every path from the root is increasing
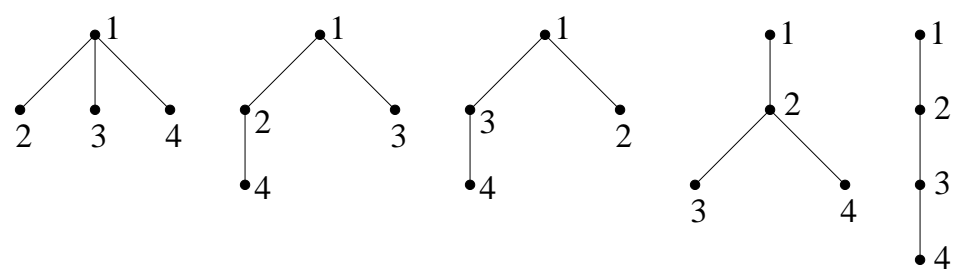

$\left(\mathrm{a}^{4}\right)$ Left factors $L$ of Dyck paths such that $L$ has $n-1$ up steps

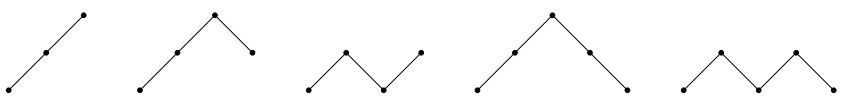


$\left(b^{4}\right)$ Dyck paths of length $2 n+2$ whose first downstep is followed by another downstep

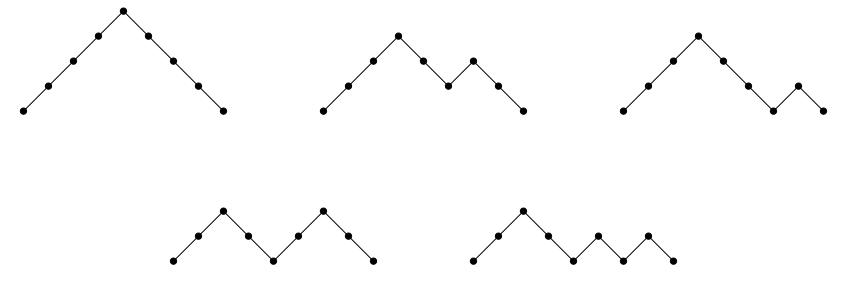

$\left(c^{4}\right)$ Dyck paths of length $2 n+2$ with no peak (an upstep followed immediately by a downstep) on the $x$-axis and having leftmost peak at height 2 or 3

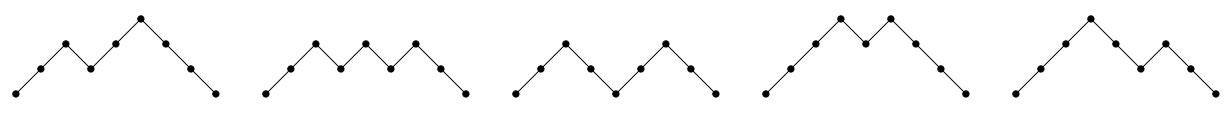

$\left(d^{4}\right)$ Dyck paths of length $2 n+2$ for which the terminal descent is of even length and all other descents (if any) to the $x$-axis are of odd length, where a descent is a maximal sequence of consecutive down steps (compare $(\mathrm{j})$ )

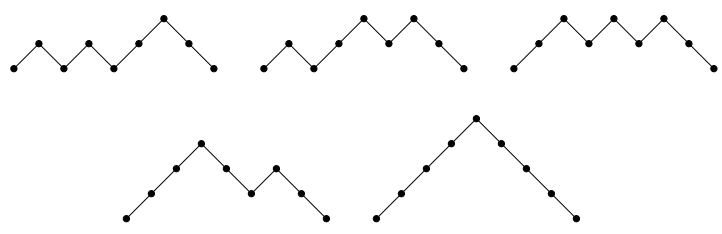

$\left(\mathrm{e}^{4}\right)$ Dyck paths of length $4 n$ such that every descent has length 2

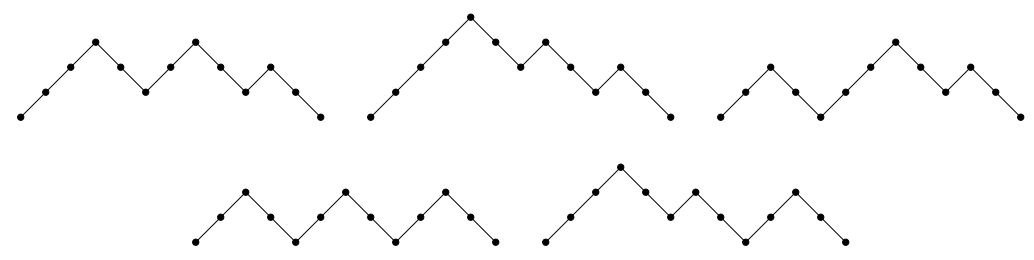

$\left(\mathrm{f}^{4}\right)$ Dyck paths with $n-1$ peaks and without three consecutive up steps or three consecutive down steps 
$\left(\mathrm{g}^{4}\right)$ Dyck paths with $n$ peaks such that there are no factors (consecutive steps) $U U U$ and $U U D D$

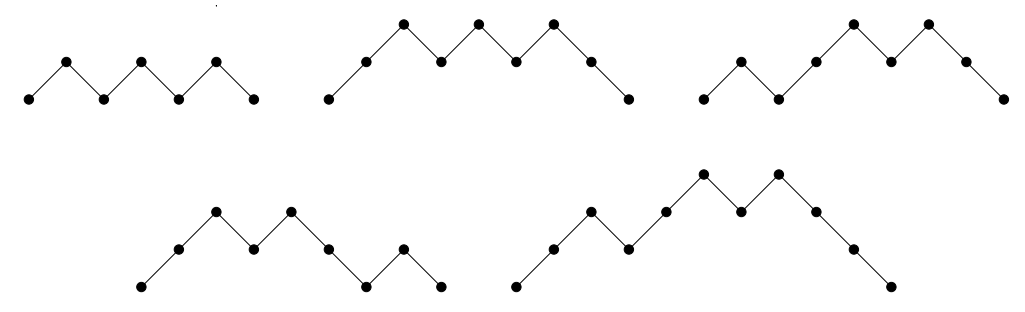

$\left(\mathrm{h}^{4}\right)$ Dyck paths of length $2 n+2$ whose second ascent (maximal sequence of consecutive up steps) has length 0 or 2

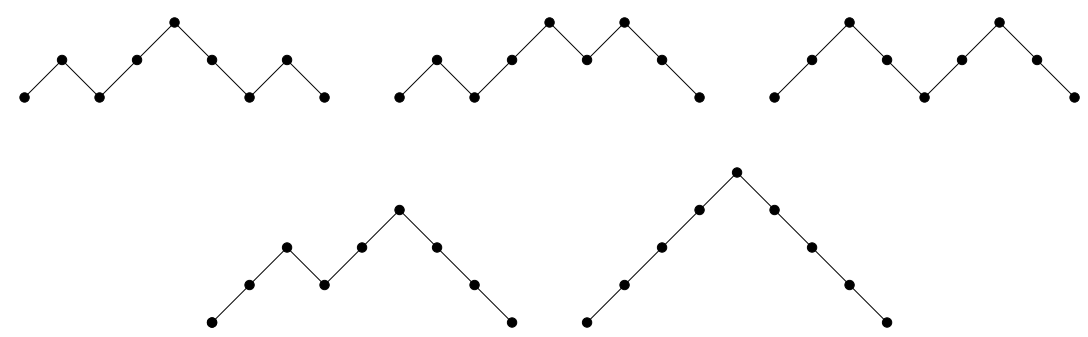

$\left(\mathrm{i}^{4}\right)$ Dyck paths $D$ from $(0,0)$ to $(2 n+2,0)$ such that there is no horizontal line segment $L$ with endpoints $(i, j)$ and $(2 n+2-i, j)$, with $i>0$, such that the endpoints lie on $P$ and no point of $L$ lies above $D$

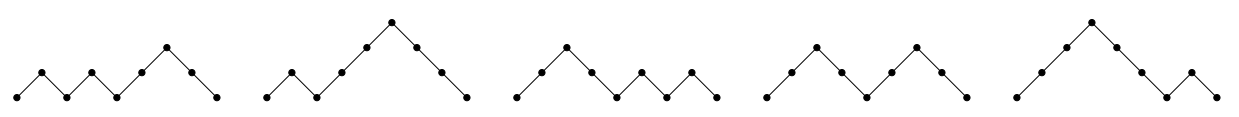

$\left(\mathrm{j}^{4}\right)$ Points of the form $(m, 0)$ on all Dyck paths from $(0,0)$ to $(2 n-2,0)$

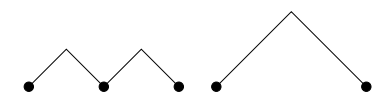


$\left(\mathrm{k}^{4}\right)$ Peaks of height one (or hills) in all Dyck paths from $(0,0)$ to $(2 n, 0)$

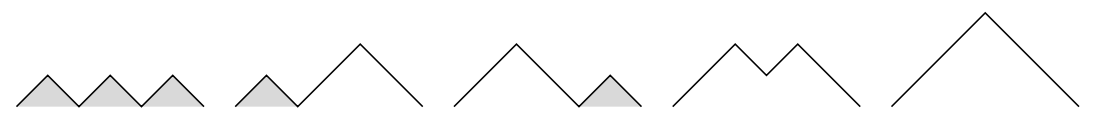

$\left(1^{4}\right)$ Decompositions $A U B D C$ of Dyck paths of length $2 n$ (regarded as a sequence of $U$ 's and $D$ 's), such that $B$ is Dyck path and such that $A$ and $C$ have the same length

\section{$\boldsymbol{U} U U D D \boldsymbol{D} \quad U \boldsymbol{U} U D D D \quad U U \boldsymbol{U} D D D$ \\ $\boldsymbol{U} U D U D \boldsymbol{D} \quad U D \boldsymbol{U} \boldsymbol{D} U D$}

$\left(\mathrm{m}^{4}\right)$ Vertices of height $n-1$ of the tree $T$ defined by the property that the root has degree 2 , and if the vertex $x$ has degree $k$, then the children of $x$ have degrees $2,3, \ldots, k+1$

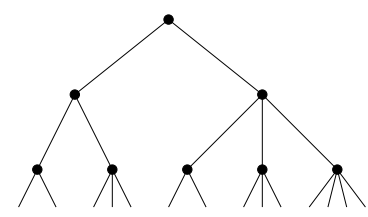

$\left(\mathrm{n}^{4}\right)$ Motzkin paths (as defined in Exercise 6.38(d), though with the typographical error $(n, n)$ instead of $(n, 0))$ from $(0,0)$ to $(n-1,0)$, with the steps $(1,0)$ colored either red or blue

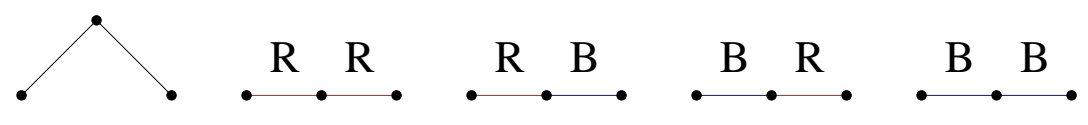

$\left(\mathrm{o}^{4}\right)$ Motzkin paths from $(0,0)$ to $(n, 0)$ with the steps $(1,0)$ colored either red or blue, and with no red $(1,0)$ steps on the $x$-axis

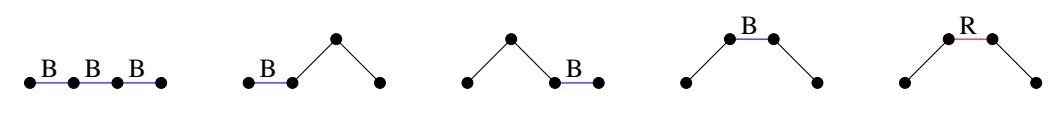


$\left(\mathrm{p}^{4}\right)$ Peakless Motzkin paths having a total of $n$ up and level steps

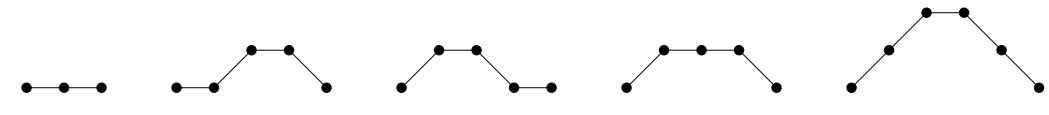

$\left(\mathrm{q}^{4}\right)$ Motzkin paths $a_{1}, \ldots, a_{2 n-2}$ from $(0,0)$ to $(2 n-2,0)$ such that each odd step $a_{2 i+1}$ is either $(1,0)$ (straight) or $(1,1)$ (up), and each even step $a_{2 i}$ is either $(1,0)$ (straight) or $(1,-1)$ (down)

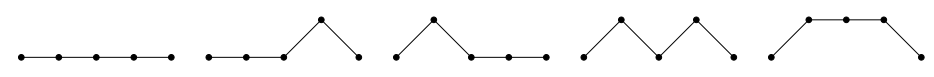

$\left(\mathrm{r}^{4}\right)$ Motzkin paths with positive integer weights on the vertices of the path such that the sum of the weights is $n$

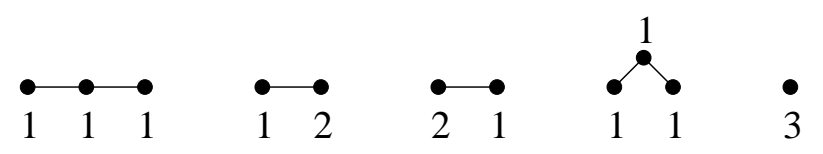

$\left(\mathrm{s}^{4}\right)$ Schröder paths as in Exercise $6.39(\mathrm{t})$ from $(0,0)$ to $(2 n, 0)$ with no peaks, i.e., no up step followed immediately by a down step

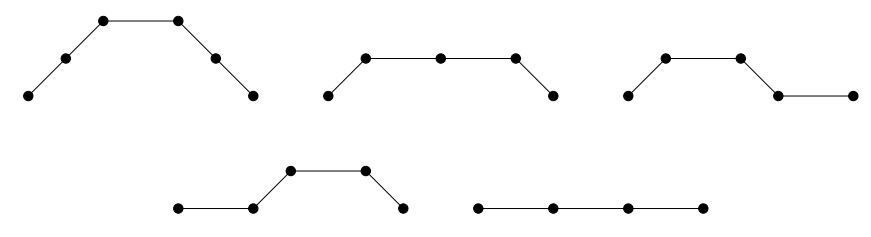

$\left(\mathrm{t}^{4}\right)$ Schröder paths as in Exercise $6.39(\mathrm{t})$ from $(0,0)$ to $(2(n-1), 0)$ with peaks allowed only at the $x$-axis

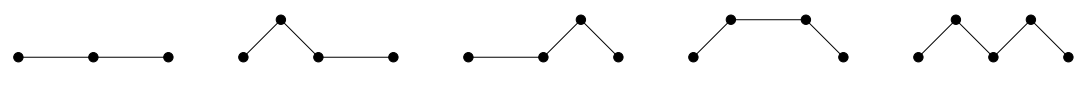

$\left(u^{4}\right)$ Schröder paths as in Exercise $6.39(t)$ from $(0,0)$ to $(2 n, 0)$ with neither peak nor level step at odd height 


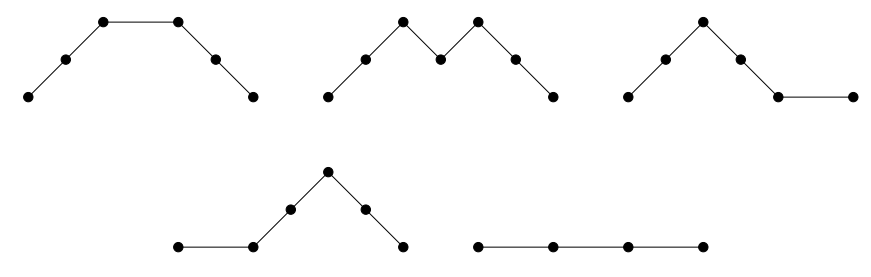

$\left(\mathrm{v}^{4}\right)$ Schröder paths as in Exercise 6.39(t) from $(0,0)$ to $(2 n-2,0)$ with no valleys, i.e., no down step followed immediately by an up step

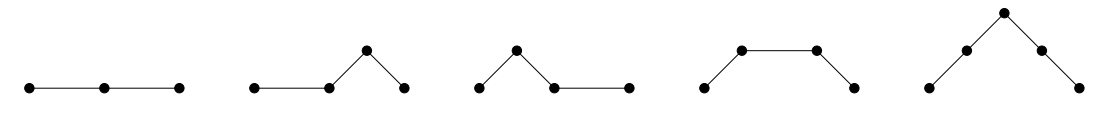

$\left(\mathrm{w}^{4}\right)$ Schröder paths as in Exercise $6.39(\mathrm{t})$ from $(0,0)$ to $(2 n-2,0)$ with no double rises, i.e., no two consecutive up steps (for $n=3$ the set of paths is coincidentally the same as for $\left.\left(t^{4}\right)\right)$

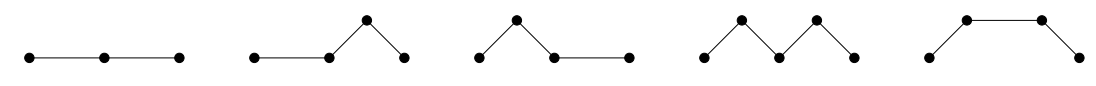

$\left(\mathrm{x}^{4}\right)$ Schröder paths as in Exercise $6.39(\mathrm{t})$ from $(0,0)$ to $(2 n, 0)$ with no level steps on the $x$-axis and no double rises

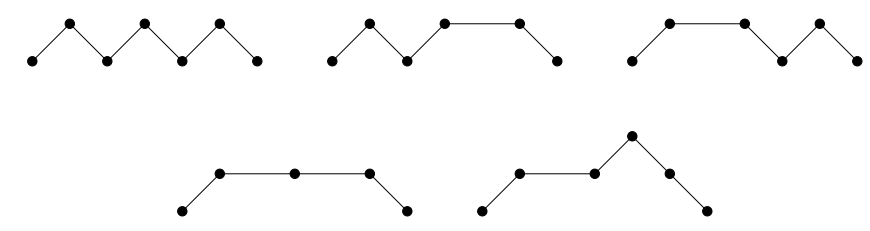

$\left(\mathrm{y}^{4}\right)$ Paths on $\mathbb{N}$ of length $n-1$ from 0 to 0 with steps $1,-1,0,0^{*}$, i.e., there are two ways to take a step by standing still (the steps in the path are given below)

$$
0,0 \quad 0^{*}, 0 \quad 0,0^{*} \quad 0^{*}, 0^{*} \quad 1,-1
$$

$\left(\mathrm{z}^{4}\right)$ Lattice paths from $(0,0)$ to $(n-1, n-1)$ with steps $(0,1),(1,0)$, and $(1,1)$, never going below the line $y=x$, such that the steps $(1,1)$ only appear on the line $y=x$ 


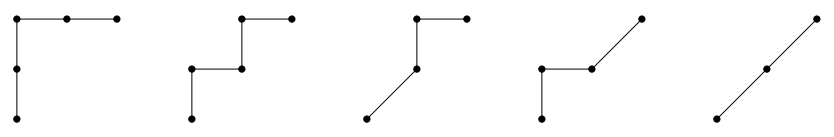

$\left(\mathrm{a}^{5}\right)$ Lattice paths from $(0,0)$ with $n-1$ up steps $(1,1)$ and $n-1$ down steps $(1,-1)$, with no peaks at height $h \leq 0$

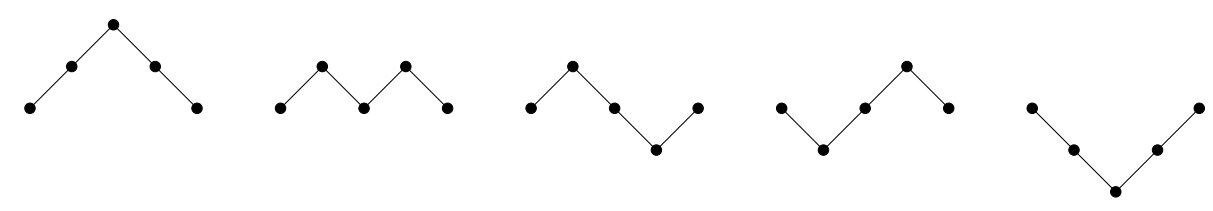

$\left(\mathrm{b}^{5}\right)$ Lattice paths from $(0,0)$ with $n$ up steps $(1,1)$ and $n$ down steps $(1,-1)$, with no peaks at height $h \leq 1$

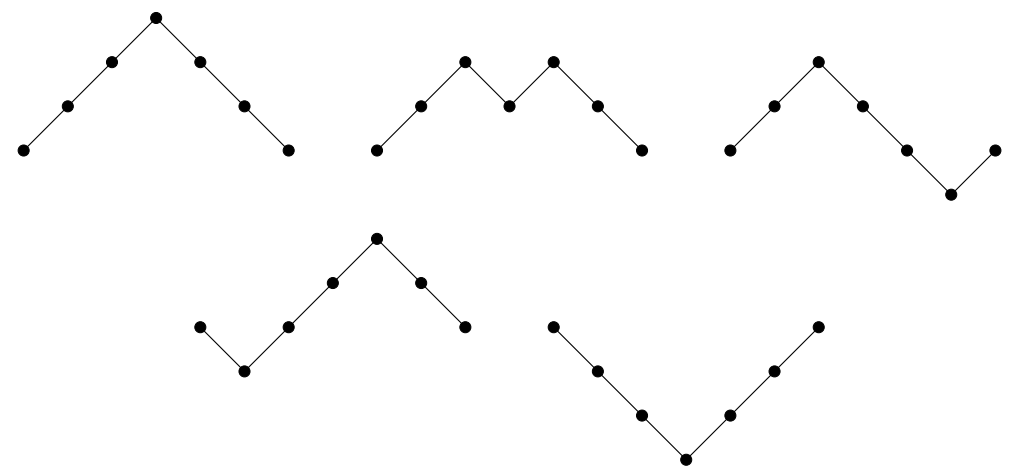

$\left(\mathrm{c}^{5}\right)$ Lattice paths from $(0,0)$ with $n+1$ steps $(1,1)$ and $n-1$ steps $(1,-1)$, such that the interior vertices with even $x$-coordinate lie strictly above the line joining the intial and terminal points

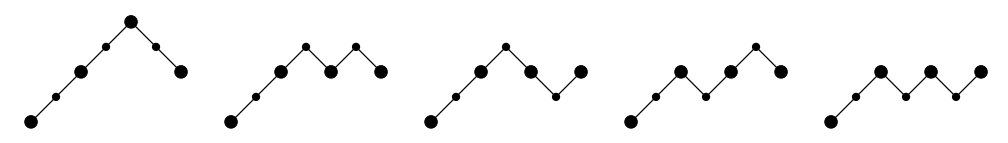

$\left(\mathrm{d}^{5}\right)$ Lattice paths of length $n-1$ from $(0,0)$ to the $x$-axis with steps $( \pm 1,0)$ and $(0, \pm 1)$, never going below the $x$-axis

$$
\begin{gathered}
(-1,0)+(-1,0) \quad(-1,0)+(1,0) \quad(0,1)+(0,-1) \\
(1,0)+(-1,0) \quad(1,0)+(1,0)
\end{gathered}
$$

$\left(\mathrm{e}^{5}\right)$ Nonnesting matchings on [2n], i.e., ways of connecting $2 n$ points in the plane lying on a horizontal line by $n$ arcs, each arc connecting 
two of the points and lying above the points, such that no arc is contained entirely below another

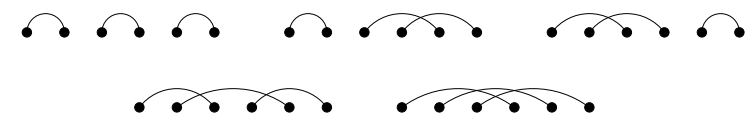

$\left(\mathrm{f}^{5}\right)$ Ways of connecting $2 n$ points in the plane lying on a horizontal line by $n$ vertex-disjoint arcs, each arc connecting two of the points and lying above the points, such that the following condition holds: for every edge $e$ let $n(e)$ be the number of edges $e^{\prime}$ that nest $e$ (i.e., $e$ lies below $\left.e^{\prime}\right)$, and let $c(e)$ be the number of edges $e^{\prime}$ that begin to the left of $e$ and that cross $e$. Then $n(e)-c(e)=0$ or 1 .

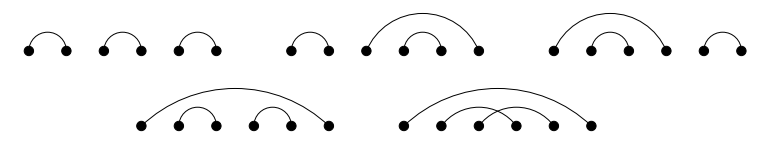

$\left(g^{5}\right)$ Ways of connecting any number of points in the plane lying on a horizontal line by nonintersecting arcs lying above the points, such that the total number of arcs and isolated points is $n-1$ and no isolated point lies below an arc

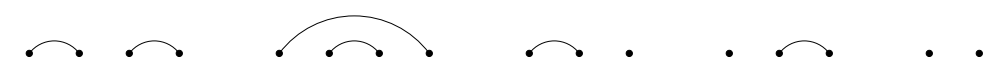

$\left(\mathrm{h}^{5}\right)$ Ways of connecting $n$ points in the plane lying on a horizontal line by noncrossing arcs above the line such that if two arcs share an endpoint $p$, then $p$ is a left endpoint of both the arcs

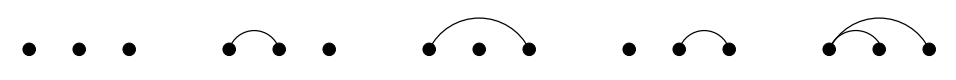

$\left(\mathrm{i}^{5}\right)$ Ways of connecting $n+1$ points in the plane lying on a horizontal line by noncrossing arcs above the line such that no arc connects adjacent points and the right endpoints of the arcs are all distinct

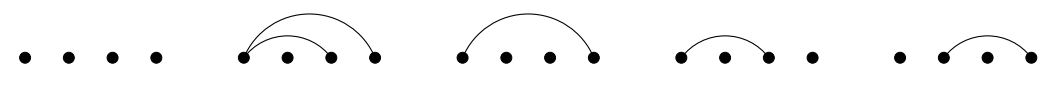


$\left(\mathrm{j}^{5}\right)$ Ways of connecting $n+1$ points in the plane lying on a horizontal line by $n$ noncrossing arcs above the line such that the left endpoints of the arcs are distinct

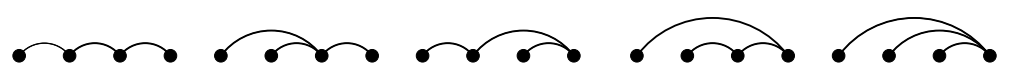

$\left(\mathrm{k}^{5}\right)$ Ways of connecting $2 n-2$ points labelled $1,2, \ldots, 2 n-2$ lying on a horizontal line by nonintersecting arcs above the line such that the left endpoint of each arc is odd and the right endpoint is even.

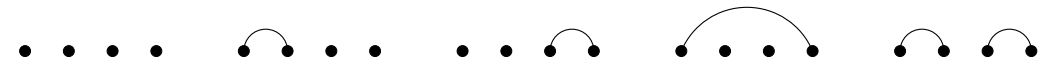

$\left(1^{5}\right)$ Noncrossing matchings of some vertex set $[k]$ into $n$ components such that no two consecutive integers form an edge

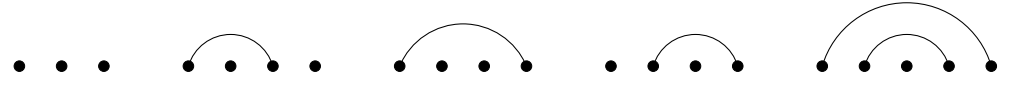

$\left(\mathrm{m}^{5}\right)$ Lattice paths in the first quadrant with $n$ steps from $(0,0)$ to $(0,0)$, where each step is of the form $( \pm 1, \pm 1)$, or goes from $(2 k, 0)$ to $(2 k, 0)$ or $(2(k+1), 0)$, or goes from $(0,2 k)$ to $(0,2 k)$ or $(0,2(k+1))$

$$
\begin{aligned}
& (0,0) \rightarrow(0,0) \rightarrow(0,0) \rightarrow(0,0) \\
& (0,0) \rightarrow(0,0) \rightarrow(1,1) \rightarrow(0,0) \\
& (0,0) \rightarrow(1,1) \rightarrow(0,0) \rightarrow(0,0) \\
& (0,0) \rightarrow(2,0) \rightarrow(1,1) \rightarrow(0,0) \\
& (0,0) \rightarrow(0,2) \rightarrow(1,1) \rightarrow(0,0)
\end{aligned}
$$

$\left(\mathrm{n}^{5}\right)$ Lattice paths from $(0,0)$ to $(n,-n)$ such that $(\alpha)$ from a point $(x, y)$ with $x<2 y$ the allowed steps are $(1,0)$ and $(0,1),(\beta)$ from a point $(x, y)$ with $x>2 y$ the allowed steps are $(0,-1)$ and $(1,-1)$, $(\gamma)$ from a point $(2 y, y)$ the allowed steps are $(0,1),(0,-1)$, and $(1,-1)$, and $(\delta)$ it is forbidden to enter a point $(2 y+1, y)$

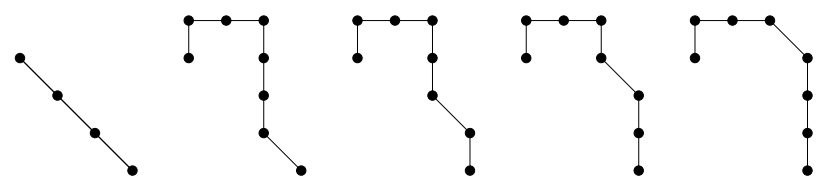


$\left(\mathrm{o}^{5}\right)$ Symmetric parallelogram polyominos (as defined in the solution to Exercise $6.19(1))$ of perimeter $4(2 n+1)$ such that the horizontal boundary steps on each level (equivalently, vertical boundary steps with fixed $x$-coordinate) form an unbroken line
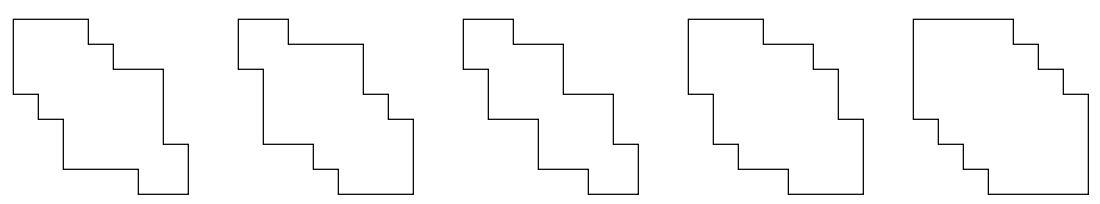

$\left(\mathrm{p}^{5}\right)$ All horizontal chords in the nonintersecting chord diagrams of (n) (with the vertices drawn so that one of the diagrams has $n$ horizontal chords)

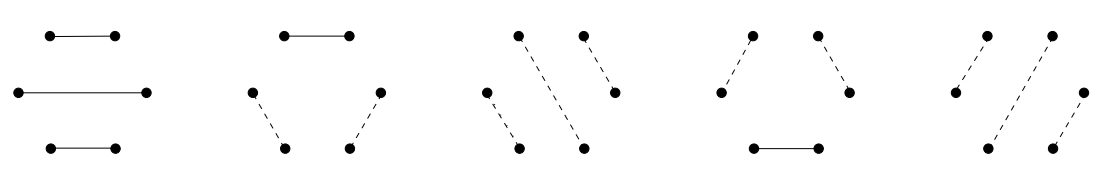

$\left(\mathrm{q}^{5}\right)$ Kepler towers with $n$ bricks, i.e., sets of concentric circles, with "bricks" (arcs) placed on each circle, as follows: the circles come in sets called walls from the center outwards. The circles (or rings) of the $i$ th wall are divided into $2^{i}$ equal arcs, numbered $1,2, \ldots, 2^{i}$ clockwise from due north. Each brick covers an arc and extends slightly beyond the endpoints of the arc. No two consecutive arcs can be covered by bricks. The first (innermost) arc within each wall has bricks at positions $1,3,5, \ldots, 2^{i}-1$. Within each wall, each brick $B$ not on the innermost ring must be supported by another brick $B^{\prime}$ on the next ring toward the center, i.e., some ray from the center must intersect both $B$ and $B^{\prime}$. Finally, if $i>1$ and the $i$ th wall is nonempty, then wall $i-1$ must also by nonempty. Figure 1 shows a Kepler tower with three walls, six rings, and 13 bricks.
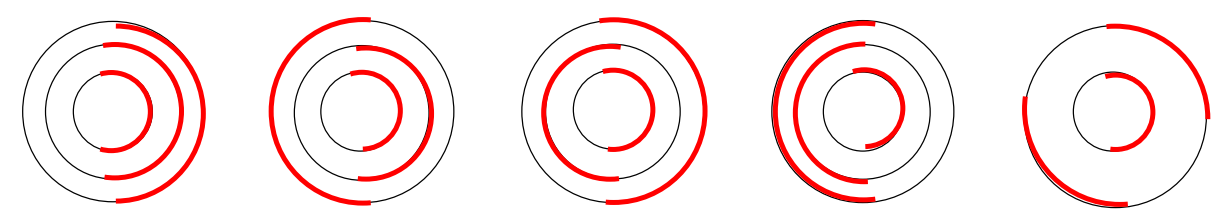


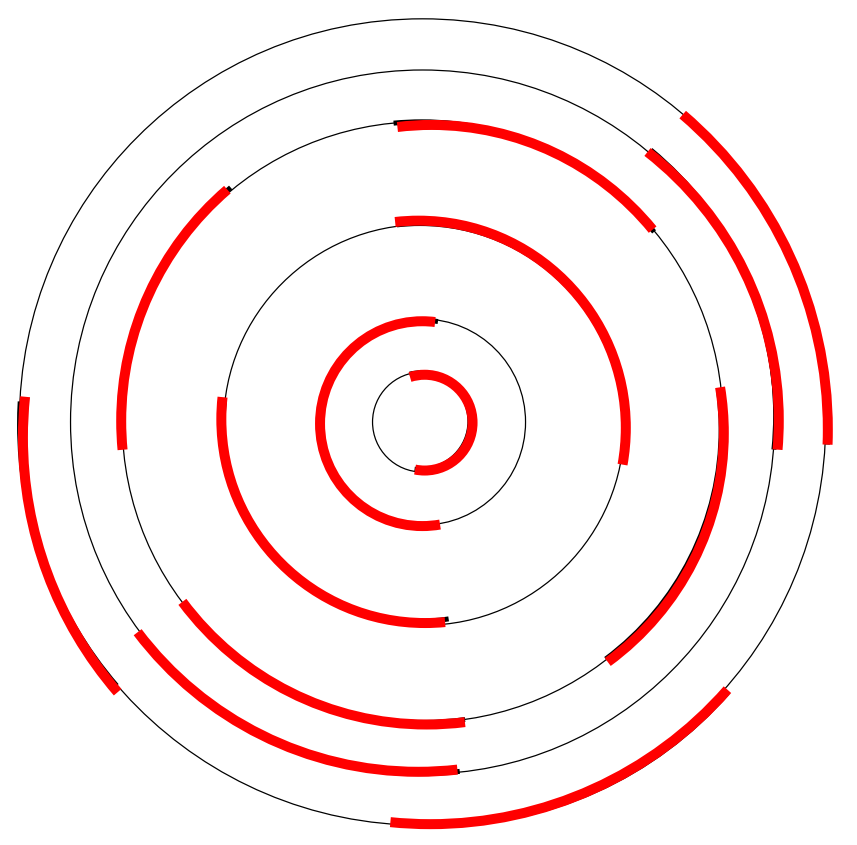

Figure 1: A Kepler tower with 3 walls, 6 rings, and 13 bricks

$\left(\mathrm{r}^{5}\right)$ Compositions of $n$ whose parts equal to $k$ are colored with one of $C_{k-1}$ colors (colors are indicated by subscripts below)

$$
1+1+1 \quad 1+2 \quad 2+1 \quad 3_{a} \quad 3_{b}
$$

$\left(\mathrm{s}^{5}\right)$ Sequences $\left(a_{1}, \ldots, a_{n}\right)$ of nonnegative integers satisfying $a_{1}+\cdots+$ $a_{i} \geq i$ and $\sum a_{j}=n$

$$
\begin{array}{lllll}
111 & 120 & 210 & 201 & 300
\end{array}
$$

$\left(\mathrm{t}^{5}\right)$ Sequences $1 \leq a_{1} \leq a_{2} \leq \cdots \leq a_{n} \leq n$ of integers with exactly one fixed point, i.e., exactly one value of $i$ for which $a_{i}=i$

\section{$\begin{array}{lllll}111 & 112 & 222 & 233 & 333\end{array}$}

$\left(u^{5}\right)$ Sequences $a_{1}, a_{2}, \ldots, a_{n}$ of positive integers such that the first appearance of $i \geq 1$ occurs before the first appearance of $i+1$, and there are no subsequences of the form $a b a b$

$\begin{array}{lllll}111 & 112 & 121 & 122 & 123\end{array}$


$\left(\mathrm{v}^{5}\right)$ Sequences $1 \leq a_{1}<a_{2}<\cdots<a_{n} \leq 2 n$ such that $a_{i} \geq 2 i$

$$
246 \quad 256 \quad 346 \quad 356 \quad 456
$$

$\left(\mathrm{w}^{5}\right)$ Sequences $1 \leq a_{1}<a_{2}<\cdots<a_{n} \leq 2 n$ such that $a_{i} \leq 2 i-1$

$\left(\mathrm{x}^{5}\right)$ Number of pairs of integer sequences $1 \leq a_{1}<a_{2}<\cdots<a_{k} \leq$ $n$ and $1 \leq b_{1}<b_{2}<\cdots<b_{k} \leq n$ for which there exists a permutation $w \in \mathfrak{S}_{n}$ whose left-to-right maxima (or records) are given by $w\left(a_{i}\right)=b_{i}$

$$
(123,123) \quad(12,13) \quad(13,23) \quad(12,23) \quad(1,3)
$$

$\left(\mathrm{y}^{5}\right)$ Sequences $a_{0}, \ldots, a_{n}$ defined recursively as follows: 1 is an allowed sequence (the case $n=0$ ). A sequence $a_{0}, \ldots, a_{n}$ is obtained from one for $n-1$ by either appending 1 at the end, or by inserting between two consecutive terms their sum

\section{$\begin{array}{lllll}1111 & 1211 & 1121 & 1231 & 1321\end{array}$}

$\left(\mathrm{z}^{5}\right)$ Sequences $a_{1}, \ldots, a_{2 n}$ of nonnegative integers with $a_{1}=1, a_{2 n}=0$ and $a_{i}-a_{i-1}= \pm 1$ :

$$
\begin{array}{lllll}
123210 & 121210 & 121010 & 101210 & 101010
\end{array}
$$

$\left(a^{6}\right)$ Sequences $w=a_{1} \cdots a_{n+1}$ of nonnegative integers such that $a_{k+1} \geq$ $a_{k}-1$ for all $1 \leq k \leq n$, and such that if $w$ contains an $i \geq 1$, then the first $i$ lies between two $i-1$ 's (not necessarily consecutively)

$$
\begin{array}{lllll}
0000 & 0010 & 0100 & 0101 & 0110
\end{array}
$$

$\left(\mathrm{b}^{6}\right)$ Sequences $a_{1}, \ldots, a_{n}$ of nonnegative integers with $a_{1}=0$ and for $2 \leq i \leq n$ :

$$
\max \left\{a_{1}, \ldots, a_{i-1}\right\}-1 \leq a_{i} \leq \operatorname{asc}\left(a_{1}, \ldots, a_{i-1}\right)+1,
$$

where $\operatorname{asc}\left(a_{1}, \ldots, a_{i-1}\right)$ denotes the number of ascents (indices $1 \leq$ $j \leq n-1$ for which $\left.a_{j}<a_{j+1}\right)$ of the sequence $\left(a_{1}, \ldots, a_{i-1}\right)$

$$
\begin{array}{lllll}
000 & 001 & 010 & 011 & 012
\end{array}
$$


$\left(c^{6}\right)$ Sequences $a_{1}, \ldots, a_{n+1}$ of nonnegative integers such that $\sum_{i=1}^{n+1} 2^{-a_{i}}=$ 1 and for all all $2 \leq j \leq n+1$ the number $2^{a_{j}} \sum_{i=1}^{j-1} 2^{-a_{i}}$ is an integer

$$
\begin{array}{lllll}
1233 & 1332 & 2222 & 2331 & 3321
\end{array}
$$

$\left(\mathrm{d}^{6}\right)$ Sequences of $n-11$ 's and any number of -1 's such that every partial sum is nonnegative

$$
1,1 \quad 1,1,-1 \quad 1,-1,1 \quad 1,1,-1,-1 \quad 1,-1,1,-1
$$

$\left(e^{6}\right)$ Subsequences of the sequence

$$
\left(a_{1}, a_{2}, \ldots, a_{2 n-2}\right)=(1,-1,1,-1, \ldots, 1,-1)
$$

that are ballot sequences (as defined in Corollary 6.2.3(ii))

$$
\emptyset \quad\left(a_{1}, a_{2}\right) \quad\left(a_{1}, a_{4}\right) \quad\left(a_{3}, a_{4}\right) \quad\left(a_{1}, a_{2}, a_{3}, a_{4}\right)
$$

$\left(\mathrm{f}^{6}\right)$ Sequences $a_{1} a_{2} \cdots a_{2 n-2}$ of $n-11$ 's and $n-1-1$ 's such that if $a_{i}=-1$ then either $a_{i+1}=a_{i+2}=\cdots=a_{2 n-2}=-1$ or $a_{i+1}+$ $a_{i+2}+\cdots+a_{i+j}>0$ for some $j \geq 1$

$$
1,1,-1,-1 \quad 1,-1,1,-1 \quad-1,1,1,-1 \quad-1,1,-1,1 \quad-1,-1,1,1
$$

$\left(\mathrm{g}^{6}\right)$ Sequences $a_{1} a_{2} \cdots a_{n}$ of integers such that $a_{1}=1, a_{n}= \pm 1, a_{i} \neq 0$, and $a_{i+1} \in\left\{a_{i}, a_{i}+1, a_{i}-1,-a_{i}\right\}$ for $2 \leq i \leq n$

$$
1,1,1 \quad 1,1,-1 \quad 1,-1,1 \quad 1,-1,-1 \quad 1,2,1
$$

$\left(\mathrm{h}^{6}\right)$ Sequences $a_{1} a_{2} \cdots a_{n}$ of nonnegative integers such that $a_{j}=\#\{i$ : $\left.i<j, a_{i}<a_{j}\right\}$ for $1 \leq j \leq n$

$$
\begin{array}{lllll}
000 & 002 & 010 & 011 & 012
\end{array}
$$

$\left(^{6}\right)$ Sequences $a_{1} a_{2} \cdots a_{n-1}$ of nonnegative integers such that each nonzero term initiates a factor (subsequence of consecutive elements) whose length is equal to its sum

$$
\begin{array}{lllll}
00 & 01 & 10 & 11 & 20
\end{array}
$$


$\left(\mathrm{j}^{6}\right)$ Sequences $a_{1} a_{2} \cdots a_{2 n+1}$ of positive integers such that $a_{2 n+1}=1$, some $a_{i}=n+1$, the first appearance of $i+1$ follows the first appearance of $i$, no two consecutive terms are equal, no pair $i j$ of integers occur more than once as a factor (i.e., as two consecutive terms), and if $i j$ is a factor then so is $j i$

\section{$\begin{array}{lllll}1213141 & 1213431 & 1232141 & 1232421 & 1234321\end{array}$}

$\left(\mathrm{k}^{6}\right)$ Sequences $\left(a_{1}, \ldots, a_{n}\right) \in \mathbb{N}^{n}$ for which there exists a distributive lattice of rank $n$ with $a_{i}$ join-irreducibles of rank $i, 1 \leq i \leq n$

\section{$\begin{array}{lllll}300 & 210 & 120 & 201 & 111\end{array}$}

$\left(1^{6}\right)$ Pairs of sequences $1 \leq i_{1}<i_{2}<\cdots<i_{k} \leq n-1$ and $2 \leq j_{1}<$ $\cdots<j_{k} \leq n$ such that $i_{r}<j_{r}$ for all $r$.

$$
(\emptyset, \emptyset) \quad(1,2) \quad(1,3) \quad(2,3) \quad(12,23)
$$

$\left(\mathrm{m}^{6}\right)$ Distinct sets $S$ obtained by starting with a permutation $w \in \mathfrak{S}_{2 n}$ and continually crossing out the smallest element and then removing the leftmost element and placing it in $S$, until no elements remain. For instance, if $w=324165$, then cross out 1 and place 3 in $S$, then cross out 2 and place 4 in $S$, and then cross out 5 and place 6 in $S$, obtaining $S=\{3,4,6\}$.

$$
\{2,4,6\}, \quad\{2,5,6\}, \quad\{3,4,6\}, \quad\{3,5,6\}, \quad\{4,5,6\}
$$

$\left(n^{6}\right)$ Ways two persons can each start with 0 and alternatingly add positive integers to their numbers so that they first have equal numbers when that number is $n$ (notation such as 1,$2 ; 4,3 ; 5,5$ means that the first person adds 1 to 0 to obtain 1 , then the second person adds 2 to 0 to obtain 2 , then the first person adds 3 to 1 to obtain 4 , etc.)

$$
3,3 \quad 2,3 ; 3 \quad 2,1 ; 3,3 \quad 1,2 ; 3,3 \quad 1,3 ; 3
$$

$\left(\mathrm{o}^{6}\right)$ Cyclic equivalence classes (or necklaces) of sequences of $n+1$ 's and $n$ 0's (one sequence from each class is shown below)

$$
\begin{array}{lllll}
1111000 & 1110100 & 1110010 & 1101100 & 1101010
\end{array}
$$


$\left(\mathrm{p}^{6}\right)$ Integer partitions that are both $n$-cores and $(n+1)$-cores, in the terminology of Exercise 7.59(d).

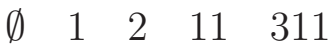

$\left(q^{6}\right)$ 231-avoiding partitions of $[n]$, i.e., partitions of $[n]$ such that if they are written with increasing entries in each block and blocks arranged in increasing order of their first entry, then the permutation of $[n]$ obtained by erasing the dividers between the blocks is 231-avoiding

\section{$1-2-3 \quad 12-3 \quad 13-2 \quad 1-23 \quad 123$}

(The only partition of [4] that isn't 231-avoiding is 134-2.)

$\left(\mathrm{r}^{6}\right)$ Equivalence classes of the equivalence relation on the set $S_{n}=$ $\left\{\left(a_{1}, \ldots, a_{n}\right) \in \mathbb{N}^{n}: \sum a_{i}=n\right\}$ generated by $(\alpha, 0, \beta) \sim(\beta, 0, \alpha)$ if $\beta$ (which may be empty) contains no 0's. For instance, when $n=7$ one equivalence class is given by

$$
\begin{gathered}
\{3010120,0301012,1200301,1012003\} . \\
\{300,030,003\}\{210,021\}\{120,012\} \quad\{201,102\}\{111\}
\end{gathered}
$$

$\left(\mathrm{s}^{6}\right)$ Pairs $(\alpha, \beta)$ of compositions of $n$ with the same number of parts, such that $\alpha \geq \beta$ (dominance order, i.e., $\alpha_{1}+\cdots+\alpha_{i} \geq \beta_{1}+\cdots+\beta_{i}$ for all $i$ )

$$
(111,111) \quad(12,12) \quad(21,21) \quad(21,12) \quad(3,3)
$$

$\left(\mathrm{t}^{6}\right)$ Indecomposable (as defined in Exercise 1.32(a) (or Exercise 1.128 in the second edition)) $w$-avoiding permutations of $[n+1]$, where $w$ is any of 321,312 , or 231 .

$$
\begin{array}{llllll}
w=321: & 4123 & 3142 & 2413 & 3412 & 2341 \\
w=312: & 4321 & 3421 & 2431 & 2341 & 3241 \\
w=231: & 4123 & 4132 & 4213 & 4312 & 4321
\end{array}
$$

$\left(u^{6}\right)$ Decomposable 213-avoiding permutations of $[n+1]$, where $w$ is either of 213 or 132

$$
\begin{array}{llllll}
w=213: & 1432 & 1423 & 1342 & 1243 & 1234 \\
w=132: & 1234 & 2134 & 2314 & 3124 & 3214
\end{array}
$$


$\left(\mathrm{v}^{6}\right)$ weak ordered partitions $(P, V, A, D)$ of $[n]$ into four blocks such that there exists a permutation $w=a_{1} a_{2} \cdots a_{n} \in \mathfrak{S}_{n}$ (with $a_{0}=$ $\left.a_{n+1}=0\right)$ satisfying

$$
\begin{aligned}
P & =\left\{i \in[n]: a_{i-1}<a_{i}>a_{i+1}\right\} \\
V & =\left\{i \in[n]: a_{i-1}>a_{i}<a_{i+1}\right\} \\
A & =\left\{i \in[n]: a_{i-1}<a_{i}<a_{i+1}\right\} \\
D & =\left\{i \in[n]: a_{i-1}>a_{i}>a_{i+1}\right\}
\end{aligned}
$$

$$
(3, \emptyset, 12, \emptyset) \quad(3, \emptyset, 1,2) \quad(23,1, \emptyset, \emptyset) \quad(3, \emptyset, 2,1) \quad(3, \emptyset, \emptyset, 12)
$$

$\left(\mathrm{w}^{6}\right)$ Factorizations $(1,2, \ldots, n+1)=\left(a_{1}, b_{1}\right)\left(a_{2}, b_{2}\right) \cdots\left(a_{n}, b_{n}\right)$ of the cycle $(1,2, \ldots, n+1)$ into $n$ transpositions $\left(a_{i}, b_{i}\right)$ such that $a_{i}<b_{i}$ for all $i$ and $a_{1} \leq a_{2} \leq \cdots \leq a_{n}$ (where we multiply permutations right-to-left)

$$
(14)(13)(12) \quad(14)(12)(23) \quad(13)(12)(34)
$$

$$
(12)(24)(23) \quad(12)(23)(34)
$$

$\left(\mathrm{x}^{6}\right)$ Sequences $a_{1} a_{2} \cdots a_{p}$ such that (i) if $u_{1}, \ldots, u_{n-1}$ is any fixed ordering (chosen at the beginning) of the adjacent transpositions $s_{i}=(i, i+1) \in \mathfrak{S}_{n}$, then $u_{a_{1}} u_{a_{2}} \cdots u_{a_{p}}$ is reduced, i.e., has $p$ inversions as a permutation in $\mathfrak{S}_{n}$, and (ii) if the descent set of the sequence $a_{1} a_{2} \cdots a_{p}$ is $1 \leq j_{1}<\cdots<j_{k} \leq n-1$, then

$$
\left\{a_{1}, \ldots, a_{j_{1}}\right\} \supset\left\{a_{j_{1}+1}, \ldots, a_{j_{2}}\right\} \supset \cdots \supset\left\{a_{j_{k}+1}, \ldots, a_{p}\right\} .
$$

For instance, if $n=10$ and $u_{i}=s_{i}$ for all $i$, then an example of a sequence being counted is 123456789124678167 . For $n=3$ and $u_{i}=s_{i}$, we get the sequences

$$
\begin{array}{lllll}
\emptyset & 1 & 2 & 12 & 121
\end{array}
$$

$\left(\mathrm{y}^{6}\right)$ Number of distinct terms (monomials) appearing in the expansion of $\prod_{i=1}^{n}\left(x_{1}+x_{2}+\cdots+x_{i}\right)$

$$
x(x+y)(x+y+z)=x^{3}+2 x^{2} y+x y^{2}+x^{2} z+x y z
$$


$\left(\mathrm{z}^{6}\right)$ Shuffles of the permutation $12 \cdots n$ with itself, i.e., permutations of the multiset $\left\{1^{2}, 2^{2}, \ldots, n^{2}\right\}$ which are a union of two disjoint subsequences $12 \cdots n$ (equivalently, there is no weakly decreasing subsequence of length three)

$$
\begin{array}{lllll}
112233 & 112323 & 121233 & 121323 & 123123
\end{array}
$$

$\left(a^{7}\right)$ Unique shuffles of the permutation $12 \cdots(n+1)$ with itself, i.e., permutations of the multiset $\left\{1^{2}, 2^{2}, \ldots,(n+1)^{2}\right\}$ which are a union of two disjoint subsequences $12 \cdots(n+1)$ in exactly one way

$$
\begin{array}{lllll}
12132434 & 12134234 & 12312434 & 12314234 & 12341234
\end{array}
$$

$\left(\mathrm{b}^{7}\right)$ Pats $w \in \mathfrak{S}_{n+1}$, defined recursively as follows: a word $a \in \mathbb{P}^{*}$ is a pat if $a$ is a singleton or can be uniquely factored $a=b c$ such that every letter of $b$ is greater than every letter of $c$, and such that the reverse of $a$ and $b$ are pats

$$
2431 \quad 3241 \quad 3412 \quad 4132 \quad 4213
$$

$\left(\mathrm{c}^{7}\right)$ Permutations of the multiset $\left\{1^{2}, 2^{2}, \ldots,(n+1)^{2}\right\}$ such that the first appearances of $1,2, \ldots, n, n+1$ occur in that order, and between the two appearances of $i$ there is exactly one $i+1$ for $i=1,2, \ldots, n$.

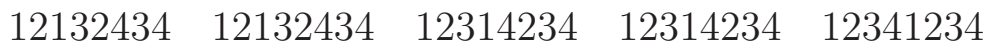

$\left(\mathrm{d}^{7}\right)$ Permutations $a_{1} \cdots a_{n} \in \mathfrak{S}_{n}$ for which there does not exist $1 \leq$ $i<j \leq n$ satisfying any of the conditions $i<j \leq a_{i}<a_{j}$ and $a_{i}<a_{j}<i<j$

\section{$\begin{array}{lllll}123 & 132 & 213 & 312 & 321\end{array}$}

$\left(\mathrm{e}^{7}\right)$ Permutations $w \in \mathfrak{S}_{n+1}$ of genus 0 (as defined in Exercise 6.38(p) below) satisfying $w(1)=1$

$1234 \quad 1243 \quad 1324 \quad 1342 \quad 1432$ 
$\left(\mathrm{f}^{7}\right)$ Permutations $w \in \mathfrak{S}_{n+1}$ of genus 0 satisfying $w(1)=2$

$$
\begin{array}{lllll}
2134 & 2143 & 2314 & 2341 & 2431
\end{array}
$$

$\left(\mathrm{g}^{7}\right)$ Permutations $w \in \mathfrak{S}_{n+2}$ of genus 0 satisfying $w(1)=3$

$$
32145 \quad 32154 \quad 32415 \quad 32451 \quad 32541
$$

$\left(\mathrm{h}^{7}\right)$ Permutations $w \in \mathfrak{S}_{n+2}$ of genus 0 satisfying $w(1)=n+2$

$$
\begin{array}{lllll}
52341 & 52431 & 53241 & 53421 & 54321
\end{array}
$$

(i $\left.{ }^{7}\right)$ Permutations $w \in \mathfrak{S}_{n}$ satisfying the following condition: let $w=$ $R_{s+1} R_{s} \cdots R_{1}$ be the factorization of $w$ into maximal ascending runs (so $s=\operatorname{des}(w)$, the number of descents of $w$ ). Let $m_{k}$ and $M_{k}$ be the smallest and largest elements in the run $R_{k}$. Let $n_{k}$ be the number of symbols in $R_{k}$ for $1 \leq k \leq s+1$; otherwise set $n_{k}=0$. Define $N_{k}=\sum_{i \leq k} n_{i}$ for all $k \in \mathbb{Z}$. Then $m_{s+1}>m_{s}>\cdots>m_{1}$ and $M_{i} \leq N_{i+1}$ for $1 \leq i \leq s+1$.

$$
\begin{array}{lllll}
123 & 213 & 231 & 312 & 321
\end{array}
$$

$\left(\mathrm{j}^{7}\right)$ Permutations $w \in \mathfrak{S}_{n}$ satisfying, in the notation of $\left(\mathrm{i}^{7}\right)$ above, $m_{s+1}>m_{s}>\cdots>m_{1}$ and $m_{i+1}>N_{i-1}+1$ for $1 \leq i \leq s$

$$
\begin{array}{lllll}
123 & 213 & 231 & 312 & 321
\end{array}
$$

$\left(\mathrm{k}^{7}\right)$ Permutations $w=a_{1} a_{2} \cdots a_{2 n+1} \in \mathfrak{S}_{2 n+1}$ that are symmetric (i.e., $\left.a_{i}+a_{2 n+2-i}=2 n+2\right)$ and 123-avoiding

$$
5764213 \quad 6574132 \quad 6754312 \quad 7564231 \quad 7654321
$$

$\left(1^{7}\right)$ Total number of fixed points (written in boldface below) of all 321-avoiding permutations $w \in \mathfrak{S}_{n}$

\section{$\begin{array}{lllll}123 & 132 & 312 & 213 & 231\end{array}$}

$\left(\mathrm{m}^{7}\right)$ 321-avoiding permutations $w \in \mathfrak{S}_{2 n+1}$ such that $i$ is an excedance of $w$ (i.e., $w(i)>i$ ) if and only if $i \neq 2 n+1$ and $w(i)-1$ is not an excedance of $w$ (so that $w$ has exactly $n$ excedances)

$$
4512736 \quad 3167245 \quad 3152746 \quad 4617235 \quad 5671234
$$


$\left(n^{7}\right)$ 321-avoiding alternating permutations in $\mathfrak{S}_{2 n}$ (for $\left.n \geq 2\right)$ or in $\mathfrak{S}_{2 n-1}$

$$
\begin{array}{rrrrr}
214365 & 215364 & 314265 & 315264 & 415263 \\
21435 & 21435 & 31425 & 31524 & 41523
\end{array}
$$

$\left(\mathrm{o}^{7}\right)$ 321-avoiding reverse alternating permutations in $\mathfrak{S}_{2 n-2}(n \geq 3)$ or in $\mathfrak{S}_{2 n-1}$

$$
\begin{array}{ccccc}
1324 & 2314 & 1423 & 2413 & 3412 \\
13254 & 23154 & 14253 & 24153 & 34152
\end{array}
$$

$\left(\mathrm{p}^{7}\right)$ 321-avoiding permutations of $[n+1]$ having first ascent at an even position

$$
\begin{array}{lllll}
2134 & 2143 & 3124 & 3142 & 4123
\end{array}
$$

$\left(q^{7}\right)$ 132-avoiding alternating permutations in $\mathfrak{S}_{2 n-1}$ or in $\mathfrak{S}_{2 n}$

$$
\begin{array}{cccccc}
32415 & 42315 & 43512 & 52314 & 53412 \\
435261 & 534261 & 546231 & 634251 & 645231
\end{array}
$$

$\left(\mathrm{r}^{7}\right)$ 132-avoiding reverse alternating permutations in $\mathfrak{S}_{2 n}$ or in $\mathfrak{S}_{2 n+1}$

$$
\begin{array}{cccccc}
342516 & 452316 & 453612 & 562314 & 563412 \\
4536271 & 5634271 & 5647231 & 6734251 & 6745231
\end{array}
$$

$\left(\mathrm{s}^{7}\right)$ 321-avoiding fixed-point-free involutions of [2n]

$$
\begin{array}{lllll}
214365 & 215634 & 341265 & 351624 & 456123
\end{array}
$$

$\left(t^{7}\right)$ 321-avoiding involutions of $[2 n-1]$ with one fixed point

$$
\begin{array}{lllll}
13254 & 14523 & 21354 & 21435 & 34125
\end{array}
$$

$\left(u^{7}\right)$ 213-avoiding fixed-point-free involutions of $[2 n]$

$$
456123 \quad 465132 \quad 564312 \quad 645231 \quad 654321
$$

$\left(\mathrm{v}^{7}\right)$ 213-avoiding involutions of $[2 n-1]$ with one fixed point

$$
14523 \quad 15432 \quad 45312 \quad 52431 \quad 54321
$$


$\left(\mathrm{w}^{7}\right)$ 3412-avoiding (or noncrossing) involutions of a subset of $[n-1]$

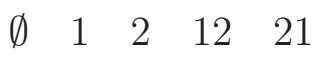

$\left(\mathrm{x}^{7}\right)$ Standard Young tableaux with at most two rows and with first row of length $n-1$

$$
\begin{array}{lllll}
12 & 12 & 13 & 12 & 13 \\
& 3 & 2 & 34 & 24
\end{array}
$$

$\left(\mathrm{y}^{7}\right)$ Standard Young tableaux with at most two rows and with first row of length $n$, such that for all $i$ the $i$ th entry of row 2 is not $2 i$

$$
\begin{array}{lllll}
123 & 123 & 124 & 123 & 124 \\
4 & 3 & 45 & 35
\end{array}
$$

$\left(\mathrm{z}^{7}\right)$ Standard Young tableaux of shape $(2 n+1,2 n+1)$ such that adjacent entries have opposite parity

$$
\begin{aligned}
& {\left[\begin{array}{ccccccc}
1 & 2 & 3 & 4 & 5 & 6 & 7 \\
8 & 9 & 10 & 11 & 12 & 13 & 14
\end{array}\right] \quad\left[\begin{array}{ccccccc}
1 & 2 & 3 & 4 & 5 & 8 & 9 \\
6 & 7 & 10 & 11 & 12 & 13 & 14
\end{array}\right]} \\
& {\left[\begin{array}{ccccccc}
1 & 2 & 3 & 4 & 5 & 10 & 11 \\
6 & 7 & 8 & 9 & 12 & 13 & 14
\end{array}\right] \quad\left[\begin{array}{ccccccc}
1 & 2 & 3 & 6 & 7 & 8 & 9 \\
4 & 5 & 10 & 11 & 12 & 13 & 14
\end{array}\right]} \\
& {\left[\begin{array}{ccccccc}
1 & 2 & 3 & 6 & 7 & 10 & 11 \\
4 & 5 & 8 & 9 & 12 & 13 & 14
\end{array}\right]}
\end{aligned}
$$

$\left(a^{8}\right)$ Arrays

$$
\left(\begin{array}{lllll}
a_{1} & a_{2} & \cdots & a_{r-1} & a_{r} \\
b_{1} & b_{2} & \cdots & b_{r-1} &
\end{array}\right)
$$

of integers, for some $r \geq 1$, such that $a_{i}>0, b_{i} \geq 0, \sum a_{i}=n$, and $b_{i}<a_{i}+b_{i-1}$ for $1 \leq i \leq r-1$ (setting $\left.b_{0}=0\right)$

$$
\left(\begin{array}{lll}
1 & 1 & 1 \\
0 & 0 &
\end{array}\right) \quad\left(\begin{array}{ll}
2 & 1 \\
0 &
\end{array}\right) \quad\left(\begin{array}{ll}
2 & 1 \\
1 &
\end{array}\right) \quad\left(\begin{array}{ll}
1 & 2 \\
0 &
\end{array}\right)\left(\begin{array}{l}
3 \\
\end{array}\right)
$$


$\left(\mathrm{b}^{8}\right)$ Plane partitions with largest part at most two and contained in a rectangle of perimeter $2(n-1)$ (including degenerate $0 \times(n-1)$ and $(n-1) \times 0$ rectangles $)$

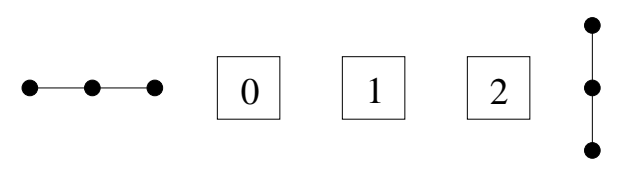

$\left(c^{8}\right)$ Ways to choose a lattice path of length $n-1$ from some point $(i, 0)$ to some point $(0, j)$, where $i, j \geq 0$, with steps $(1,0)$ and $(0,-1)$, and then coloring either red or blue some of the unit coordinate squares (cells) below the path and in the first quadrant, satisfying:

- There is no colored cell below a red cell.

- There is no colored cell to the left of a blue cell.

- Every uncolored cell is either below a red cell or to the left of a blue cell.

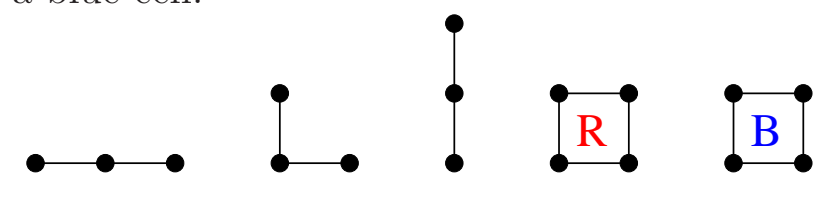

$\left(\mathrm{d}^{8}\right)$-element subsets $S$ of $\mathbb{N} \times \mathbb{N}$ satisfying the following three conditions: (a) there are no gaps in row 0, i.e., if $(k, 0) \in S$ and $k>0$, then $(k-1,0) \in S$, (b) every nonempty column has a row 0 element, i.e., if $(k, i) \in S$ then $(k, 0) \in S$, and (c) the number of gaps in column $k$ is at most the number of elements in column $k+1$, i.e., if $G_{k}=\{(k, i) \notin S:(k, j) \in S$ for some $j>i\}$ and $S_{k}=\{i:(k, i) \in S\}$, then $\# S_{k} \geq \# G_{k}$

$$
\{00,01,02\}\{00,02,10\}\{00,10,11\}\{00,01,10\}\{00,10,20\}
$$

$\left(\mathrm{e}^{8}\right)$ Triples $(A, B, C)$ of pairwise disjoint subsets of $[n-1]$ such that $\# A=\# B$ and every element of $A$ is less than every element of $B$

$$
(\emptyset, \emptyset, \emptyset) \quad(\emptyset, \emptyset, 1) \quad(\emptyset, \emptyset, 2) \quad(\emptyset, \emptyset, 12) \quad(1,2, \emptyset)
$$

$\left(\mathrm{f}^{8}\right)$ Subsets $S$ of $\mathbb{N}$ such that $0 \in S$ and such that if $i \in S$ then $i+n, i+n+1 \in S$

$$
\mathbb{N}, \quad \mathbb{N}-\{1\}, \quad \mathbb{N}-\{2\}, \quad \mathbb{N}-\{1,2\}, \quad \mathbb{N}-\{1,2,5\}
$$


(g8) Maximal chains $\emptyset=S_{0} \subset S_{1} \subset \cdots \subset S_{n}=[n]$ of subsets of $[n]$ such that $S_{i}-S_{i-1}=\{m\}$ if and only if $m$ was deleted from the rightmost maximal set of consecutive integers contained in $S_{i}$

$$
\begin{gathered}
\emptyset \subset 1 \subset 12 \subset 123, \emptyset \subset 2 \subset 12 \subset 123, \emptyset \subset 1 \subset 13 \subset 123 \\
\emptyset \subset 2 \subset 23 \subset 123, \emptyset \subset 3 \subset 23 \subset 123
\end{gathered}
$$

$\left(\mathrm{h}^{8}\right)(n+1)$-element multisets on $\mathbb{Z} / n \mathbb{Z}$ whose elements sum to 0

$$
\begin{array}{lllll}
0000 & 0012 & 0111 & 0222 & 1122
\end{array}
$$

$\left(\mathrm{i}^{8}\right)$ Ways to write $(1,1, \ldots, 1,-n) \in \mathbb{Z}^{n+1}$ as a sum of vectors $e_{i}-e_{i+1}$ and $e_{j}-e_{n+1}$, without regard to order, where $e_{k}$ is the $k$ th unit coordinate vector in $\mathbb{Z}^{n+1}$ :

$$
\begin{gathered}
(1,-1,0,0)+2(0,1,-1,0)+3(0,0,1,-1) \\
(1,0,0,-1)+(0,1,-1,0)+2(0,0,1,-1) \\
(1,-1,0,0)+(0,1,-1,0)+(0,1,0,-1)+2(0,0,1,-1) \\
(1,-1,0,0)+2(0,1,0,-1)+(0,0,1,-1) \\
(1,0,0,-1)+(0,1,0,-1)+(0,0,1,-1)
\end{gathered}
$$

$\left(j^{8}\right)$ Horizontally convex polyominoes (as defined in Example 4.7.18 of the first edition and Section 4.7.5 of the second edition) of width $n+1$ such that each row begins strictly to the right of the beginning of the previous row and ends strictly to the right of the end of the previous row
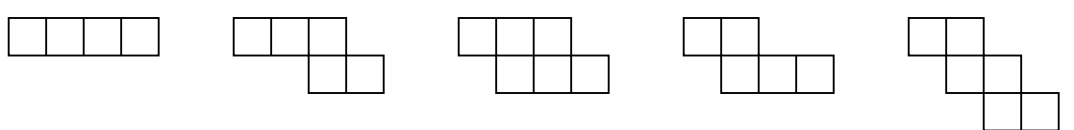

$\left(\mathrm{k}^{8}\right)$ tilings of the staircase shape $(n, n-1, \ldots, 1)$ with $n$ rectangles
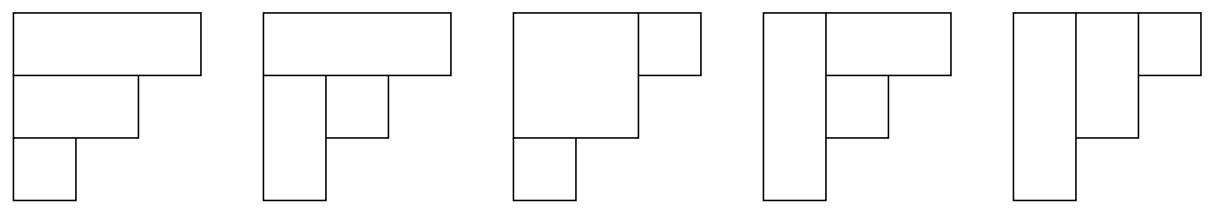


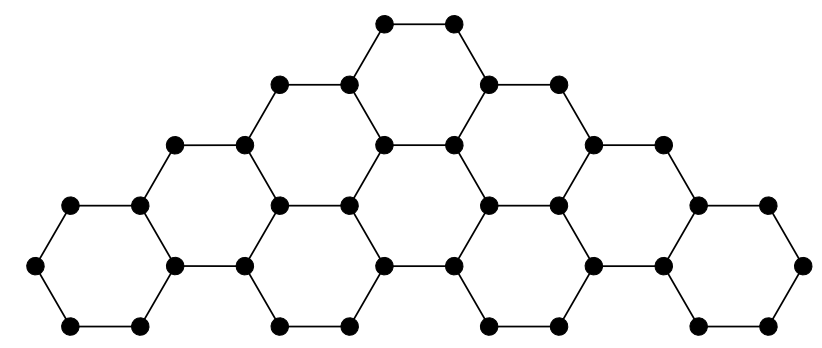

Figure 2: The triangular benzenoid graph $T_{4}$

$\left(1^{8}\right)$ Complete matchings of the triangular benzenoid graph $T_{n-1}$ of order $n-1$. The graph $T_{n}$ is a planar graph whose bounded regions are hexagons, with $i$ hexagons in row $i$ and $n$ rows in all, as illustrated for $n=4$ in Figure 2 .

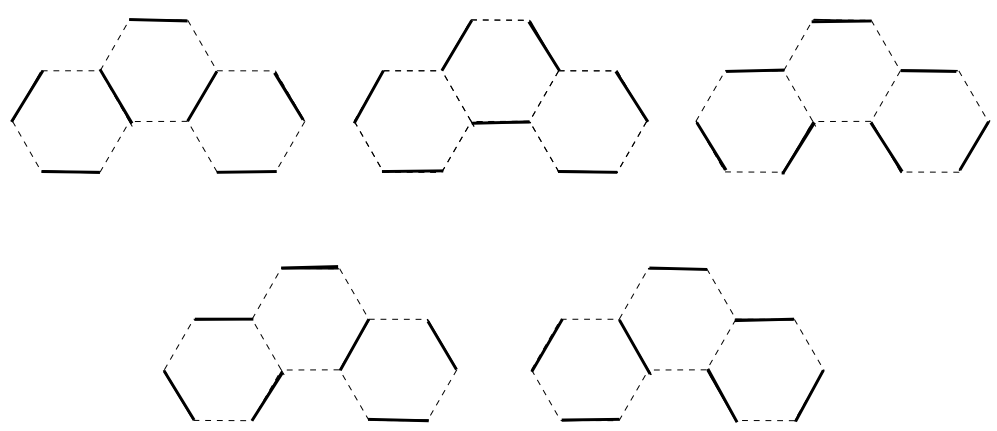

$\left(\mathrm{m}^{8}\right)$ Nonisomorphic $n$-element posets with no induced subposet isomorphic to $\mathbf{1}+\mathbf{2}$ or the fence $Z_{4}$ of Exercise 3.23 (compare (ddd))

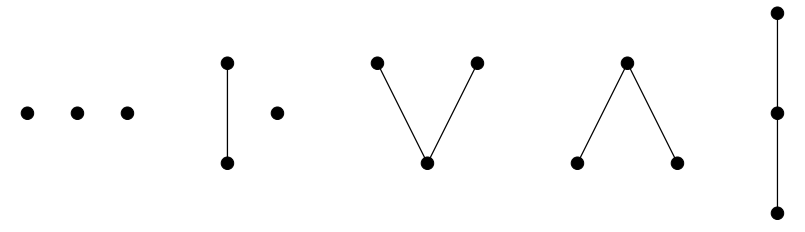

$\left(\mathrm{n}^{8}\right)$ Nonisomorphic $2(n+1)$-element posets that are a union of two chains, that are not a (nontrivial) ordinal sum, and that have a nontrivial automorphism (compare (eee)) 

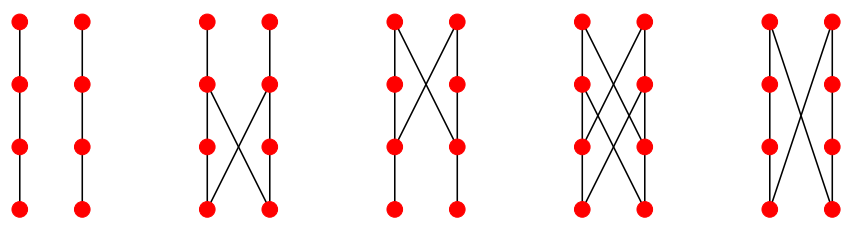

$\left(\mathrm{o}^{8}\right)$ Natural partial orderings $<_{P}$ of $[n]$ such that if $i<_{P} k$ and $i<_{\mathbb{Z}}$ $j<_{\mathbb{Z}} k$, then $i<_{P} j$

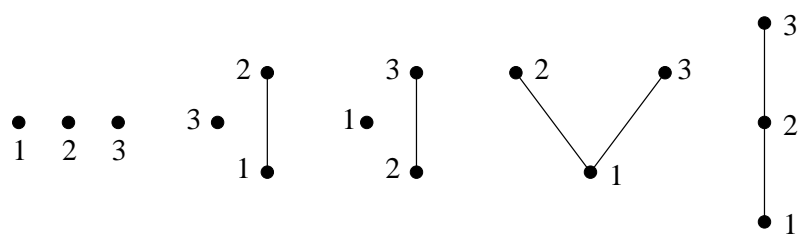

$\left(\mathrm{p}^{8}\right)$ Natural partial orderings $<_{P}$ of $[n]$ such that if $i<_{\mathbb{Z}} j<_{P} k$ then $i<_{P} k$, and dually if $i>_{\mathbb{Z}} j>_{P} k$ then $i>_{P} k$

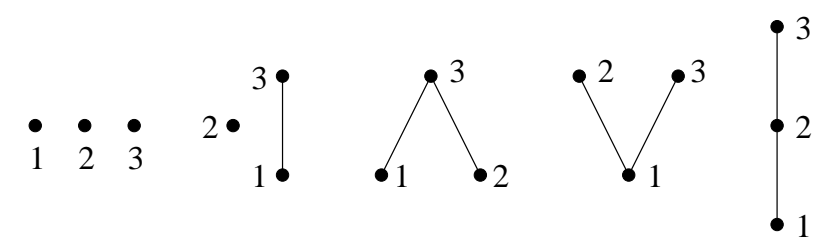

$\left(q^{8}\right)$ Permutations $w \in W\left(\mathfrak{S}_{n}\right)$, the weak (Bruhat) order of $\mathfrak{S}_{n}$, for which the interval $[\hat{0}, w]$ is a distributive lattice.

$$
\begin{array}{lllll}
123 & 132 & 213 & 231 & 312
\end{array}
$$

$\left(\mathrm{r}^{8}\right)$ Subsets $S$ of the order ideal $I_{n}=\{(i, j): i+j \leq n-2\}$ of $\mathbb{N} \times \mathbb{N}$ such that for all $(i, j) \in I$ we have $\#\left(S \cap V_{(i, j)}\right) \leq n-1-i-j$, with equality if and only if $(i, j) \in S$ (where $V_{(i, j)}=\left\{(h, k) \in I_{n}\right.$ : $(h, k) \geq(i, j)\})$

$$
\emptyset\{10\} \quad\{01\} \quad\{00,10\} \quad\{00,01\}
$$

$\left(\mathrm{s}^{8}\right)$ Isomorphism classes of ordered pairs $(S, R)$ of binary relations on $[n]$ such that $S$ and $R$ are irreflexive (i.e., don't contain any $(i, i)$ ) and transitive, such that $\bar{R} \cup \bar{S}=[n]^{2}-\{(i, i): i \in[n]\}, \bar{R} \cap \bar{S}=\emptyset$, and $S \circ R \subseteq R$. Here

$$
\begin{aligned}
\bar{T} & =T \cup\{(j, i):(i, j) \in T\} \\
S \circ R & =\{(i, k): \exists j(i, j) \in S,(j, k) \in R\} .
\end{aligned}
$$


Two pairs $(S, R)$ and $\left(S^{\prime}, R^{\prime}\right)$ are considered isomorphic if there is a bijection $f:[n] \rightarrow[n]$ inducing bijections $S \rightarrow S^{\prime}$ and $R \rightarrow R^{\prime}$.

$$
\begin{gathered}
(\emptyset,\{12,13,23\}) \quad(\{12\},\{13,23\}) \quad(\{23\},\{12,13\}) \\
(\{13,23\},\{12\}) \quad(\{12,13,23\}, \emptyset)
\end{gathered}
$$

$\left(\mathrm{t}^{8}\right) n \times n \mathbb{N}$-matrices $M=\left(m_{i j}\right)$ where $m_{i j}=0$ unless $i=n$ or $i=j$ or $i=j-1$, with row and column sum vector $(1,2, \ldots, n)$

$$
\left[\begin{array}{lll}
1 & 0 & 0 \\
0 & 2 & 0 \\
0 & 0 & 3
\end{array}\right] \quad\left[\begin{array}{lll}
0 & 1 & 0 \\
0 & 1 & 1 \\
1 & 0 & 2
\end{array}\right] \quad\left[\begin{array}{lll}
1 & 0 & 0 \\
0 & 1 & 1 \\
0 & 1 & 2
\end{array}\right] \quad\left[\begin{array}{lll}
1 & 0 & 0 \\
0 & 0 & 2 \\
0 & 2 & 1
\end{array}\right] \quad\left[\begin{array}{lll}
0 & 1 & 0 \\
0 & 0 & 2 \\
1 & 1 & 1
\end{array}\right]
$$

$\left(u^{8}\right)(n+1) \times(n+1)$ alternating sign matrices (i.e., matrices with entries $0, \pm 1$ such that every row and column sum is 1 , and the nonzero entries of each row alternate between 1 and -1 ) such that the rightmost 1 in row $i+1 \geq 2$ occurs to the right of the leftmost 1 in row $i$

$$
\begin{aligned}
& {\left[\begin{array}{llll}
1 & 0 & 0 & 0 \\
0 & 1 & 0 & 0 \\
0 & 0 & 1 & 0 \\
0 & 0 & 0 & 1
\end{array}\right] \quad\left[\begin{array}{rrrr}
0 & 1 & 0 & 0 \\
1 & -1 & 1 & 0 \\
0 & 1 & 0 & 0 \\
0 & 0 & 0 & 1
\end{array}\right] \quad\left[\begin{array}{rrrr}
0 & 1 & 0 & 0 \\
0 & 0 & 1 & 0 \\
1 & -1 & 0 & 1 \\
0 & 1 & 0 & 0
\end{array}\right]} \\
& {\left[\begin{array}{rrrr}
0 & 0 & 1 & 0 \\
1 & 0 & -1 & 1 \\
0 & 1 & 0 & 0 \\
0 & 0 & 1 & 0
\end{array}\right]\left[\begin{array}{rrrr}
0 & 0 & 1 & 0 \\
0 & 1 & -1 & 1 \\
1 & -1 & 1 & 0 \\
0 & 1 & 0 & 0
\end{array}\right]}
\end{aligned}
$$

$\left(\mathrm{v}^{8}\right)$ Bounded regions into which the cone $x_{1} \geq x_{2} \geq \cdots \geq x_{n+1}$ in $\mathbb{R}^{n+1}$ is divided by the hyperplanes $x_{i}-x_{j}=1,1 \leq i<j \leq n+1$ (compare (1ll), which illustrates the case $n=2$ of the present item)

$\left(\mathrm{w}^{8}\right)$ Number of distinct volumes of the sets

$$
X_{w}=\left\{x \in B: x_{w(1)}<x_{w(2)}<\cdots<x_{w(n)}\right\},
$$

where for fixed $0<a_{1}<a_{2}<\cdots<a_{n}$ we define

$$
\begin{gathered}
B=\left[0, a_{1}\right] \times\left[0, a_{2}\right] \times \cdots \times\left[0, a_{n}\right] \subset \mathbb{R}^{n} \\
\operatorname{vol}\left(X_{231}\right)=\operatorname{vol}\left(X_{321}\right), \operatorname{vol}\left(X_{312}\right), \operatorname{vol}\left(X_{213}\right), \operatorname{vol}\left(X_{132}\right), \operatorname{vol}\left(X_{123}\right)
\end{gathered}
$$


$\left(\mathrm{x}^{8}\right)$ Extreme rays of the closed convex cone generated by all flag $f$ vectors (i.e., the functions $\beta(P, S)$ of Section 3.12) of graded posets of rank $n$ with $\hat{0}$ and $\hat{1}$ (the vectors below lie on the extreme rays, with the coordinates $\emptyset,\{1\},\{2\},\{1,2\}$ in that order)

$$
(0,0,0,1) \quad(0,0,1,1) \quad(0,1,0,0) \quad(0,1,1,1) \quad(1,1,1,1)
$$

$\left(y^{8}\right)$ Number of elements, when written with the maximum possible number of $x$ 's, with $n x$ 's in the free near semiring $N_{1}$ on the generator $x$. (A near semiring is a set with two associative operations + and $\cdot \operatorname{such}$ that $(a+b) \cdot c=a \cdot c+b \cdot c$. Neither operation is required to be commutative. Thus $(x+x) \cdot x$ contributes to $C_{4}$ since it can be written as $x \cdot x+x \cdot x$.)

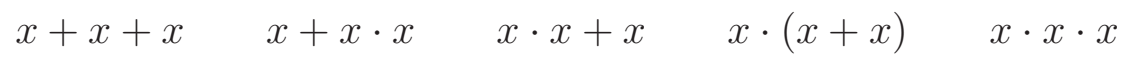

6.25 (algebraic interpretations of Catalan numbers)

(j) Degree of the Grassmannian $G(2, n+2$ ) (as a projective variety under the usual Plücker embedding) of 2-dimensional planes in $\mathbb{C}^{n+2}$

(k) Dimension (as a $\mathbb{Q}$-vector space) of the ring $\mathbb{Q}\left[x_{1}, \ldots, x_{n}\right] / Q_{n}$, where $Q_{n}$ denotes the ideal of $\mathbb{Q}\left[x_{1}, \ldots, x_{n}\right]$ generated by all quasisymmetric functions in the variables $x_{1}, \ldots, x_{n}$ with 0 constant term

(l) Multiplicity of the point $X_{w_{0}}$ in the Schubert variety $\Omega_{w}$ of the flag manifold $\operatorname{GL}(n, \mathbb{C}) / B$, where $w_{0}=n, n-1, \ldots, 1$ and $w=$ $n, 2,3, \ldots, n-2, n-1,1$

(m) Conjugacy classes of elements $A \in \operatorname{SL}(n, \mathbb{C})$ such that $A^{n+1}=1$

(n) Number of nonvanishing minors of a generic $n \times n$ upper triangular matrix $A$ (over $\mathbb{C}$, say)

(o) Dimension of the vector space spanned by the products of complementary minors of an $n \times n$ matrix $X=\left(x_{i j}\right)$ of indeterminates. For instance, when $n=3$ there are ten such products: $\operatorname{det}(A)$ itself (a product of the empty minor with the full minor) and nine products of $1 \times 1$ minors with $2 \times 2$ minors, a typical one being $x_{11}\left(x_{22} x_{33}-x_{23} x_{32}\right)$. 
(p) The matrix entry $\left(A^{2 n}\right)_{11}$, where $A=\left(a_{i j}\right)_{i, j \geq 1}$ is the tridiagonal matrix defined by $a_{i, i-1}=a_{i, i+1}=1$, and $a_{i j}=0$ otherwise

6.38 (combinatorial interpretations of Motzkin numbers)

(n) 2143-avoiding involutions (or vexillary involutions) in $\mathfrak{S}_{n}$

(o) Binary trees with $n+1$ vertices such that every non-endpoint vertex has a nonempty right subtree

(p) Permutations $w \in \mathfrak{S}_{n}$ such that both $w$ and $w^{-1}$ have genus 0 , where a permutation $w \in \mathfrak{S}_{n}$ has genus 0 if the total number of cycles of $w$ and $w^{-1}(1,2, \ldots, n)$ is $n+1$ (the largest possible)

6.39 (combinatorial interpretations of Schröder numbers)

(t) $r_{n}$ is the number of paths from $(0,0)$ to $(2 n, 0)$ with steps $(1,1)$, $(1,-1)$, and $(2,0)$, never passing below the $x$-axis.

(u) $s_{n}$ is the number of paths in (t) with no level steps on the $x$-axis.

(v) Given $n$ points $p_{1}, \ldots, p_{n}$ in the interior of a rectangle $R$ with sides parallel to the $x$ and $y$-axes, such that no two of the points are parallel to the $x$-axis or $y$-axis, $r_{n}$ is the number of rectangulations (ways to divide $R$ into rectangles) so that every $p_{i}$ lies on an edge of some rectangle, and so that the process of dividing $R$ can be carried out in $n$ steps, each step dividing some rectangle into two. Such rectangulations are called guillotine rectangulations. The total number of rectangles into which $R$ is divided will be $n+1$. Figure $3(\mathrm{a})$ shows the $r_{2}=6$ ways when $n=2$, while Figure 3(b) shows a non-guillotine rectangulation.

(w) $r_{n}$ is the number of $(n+1) \times(n+1)$ alternating sign matrices $A$ (defined in Exercise 6.19 $\left(\mathrm{u}^{8}\right)$ ) such that we never have $A_{i i^{\prime}}=$ $A_{j j^{\prime}}=A_{k k^{\prime}}=1$, where $i<j<k$ and $i^{\prime}<k^{\prime}<j^{\prime}$ (such matrices are called 132-avoiding alternating sign matrices)

(x) $r_{n-1}$ is the number of permutations $w \in \mathfrak{S}_{n}$ such that there exist real polynomials $p_{1}(x), \ldots, p_{n}(x)$, all vanishing at $x=0$, with the following property: if $p_{1}(x)<p_{2}(x)<\cdots<p_{n}(x)$ for small (i.e., sufficiently close to 0) $x<0$, then $p_{w(1)}(x)<p_{w(2)}(x)<\cdots<$ $p_{w(n)}(x)$ for small $x>0$. 

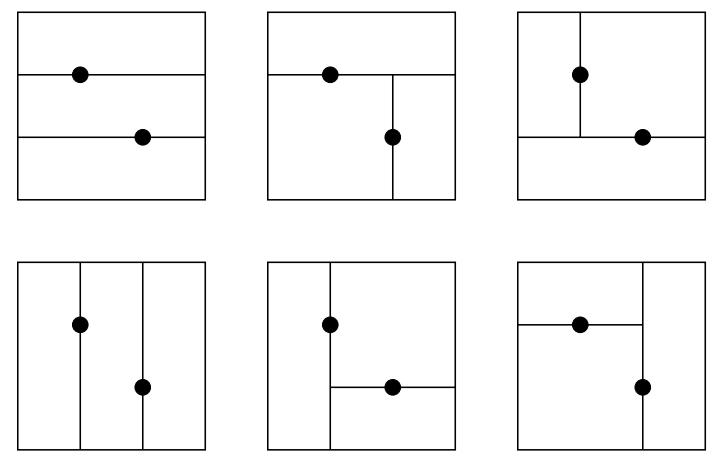

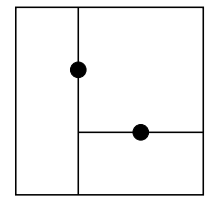

(a)

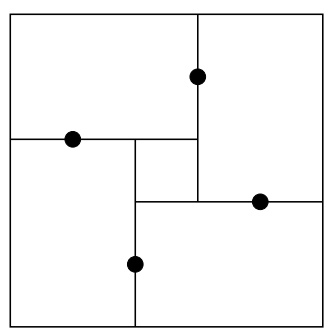

(b)

Figure 3: Dividing a rectangle into rectangles

6.C1 [3] Show that

$$
C_{n-1}=\sum \prod_{i=1}^{k}\left(\left(\begin{array}{c}
a_{i-1}+a_{i}-1 \\
a_{i}
\end{array}\right)-1\right),
$$

where the sum is over all compositions $a_{0}+a_{1}+\cdots+a_{k}=n$. The composition with $k=0$ contributes 1 to the sum.

6.C2 (a) [3] Let $a_{i, j}(n)$ (respectively, $\bar{a}_{i, j}(n)$ ) denote the number of walks in $n$ steps from $(0,0)$ to $(i, j)$, with steps $( \pm 1,0)$ and $(0, \pm 1)$, never touching a point $(-k, 0)$ with $k \geq 0$ (respectively, $k>0$ ) once leaving the starting point. Show that

$$
\begin{aligned}
a_{0,1}(2 n+1) & =4^{n} C_{n} \\
a_{1,0}(2 n+1) & =C_{2 n+1} \\
a_{-1,1}(2 n) & =\frac{1}{2} C_{2 n} \\
a_{1,1}(2 n) & =4^{n-1} C_{n}+\frac{1}{2} C_{2 n} \\
\bar{a}_{0,0}(2 n) & =2 \cdot 4^{n} C_{n}-C_{2 n+1} .
\end{aligned}
$$


(b) [3] Show that for $i \geq 1$ and $n \geq i$,

$$
\begin{aligned}
a_{-i, i}(2 n) & =\frac{i}{2 n} \frac{\left(\begin{array}{c}
2 i \\
i
\end{array}\right)\left(\begin{array}{c}
n+i \\
2 i
\end{array}\right)\left(\begin{array}{c}
4 n \\
2 n
\end{array}\right)}{\left(\begin{array}{c}
2 n+2 i \\
2 i
\end{array}\right)} \\
a_{i, i}(2 n) & =a_{-i,-i}+4^{n} \frac{i}{n}\left(\begin{array}{c}
2 i \\
i
\end{array}\right)\left(\begin{array}{c}
2 n \\
n-i
\end{array}\right) .
\end{aligned}
$$

(c) [3] Let $b_{i, j}(n)$ (respectively, $\left.\bar{b}_{i, j}(n)\right)$ denote the number of walks in $n$ steps from $(0,0)$ to $(i, j)$, with steps $( \pm 1, \pm 1)$, never touching a point $(-k, 0)$ with $k \geq 0$ (respectively, $k>0$ ) once leaving the starting point. Show that

$$
\begin{aligned}
b_{1,1}(2 n+1) & =C_{2 n+1} \\
b_{-1,1}(2 n+1) & =2 \cdot 4^{n} C_{n}-C_{2 n+1} \\
b_{0,2}(2 n) & =C_{2 n} \\
b_{2 i, 0}(2 n) & =\frac{i}{n}\left(\begin{array}{c}
2 i \\
i
\end{array}\right)\left(\begin{array}{c}
2 n \\
n-i
\end{array}\right) 4^{n-i}, i \geq 1 \\
\bar{b}_{0,0}(2 n) & =4^{n} C_{n} .
\end{aligned}
$$

(d) $[3-]$ Let

$$
f(n)=\sum_{P}(-1)^{w(P)}
$$

where (i) $P$ ranges over all lattice paths in the plane with $2 n$ steps, from $(0,0)$ to $(0,0)$, with steps $( \pm 1,0)$ and $(0, \pm 1)$, and (ii) $w(P)$ denotes the winding number of $P$ with respect to the point $\left(\frac{1}{2}, \frac{1}{2}\right)$. Show that $f(n)=4^{n} C_{n}$.

6.C3 [3] A rooted planar map is a planar embedding of an (unlabelled) connected planar graph rooted at a flag, i.e, at a triple $(v, e, f)$ where $v$ is a vertex, $e$ is an edge incident to $v$, and $f$ is a face incident to $e$. Two rooted planar maps $G$ and $H$ are considered the same if, regarding them as being on the 2-sphere $\mathbb{S}^{2}$, there is a flag-preserving homeomorphism of $\mathbb{S}^{2}$ that takes $G$ to $H$. Equivalently, a rooted planar map may be regarded as a planar embedding of a connected planar graph in which a single edge on the outer face is directed in a counterclockwise direction. (The outer face is the root face, and the tail of the root edge is the root vertex.) Figure 4 shows the nine rooted planar maps with two edges. 


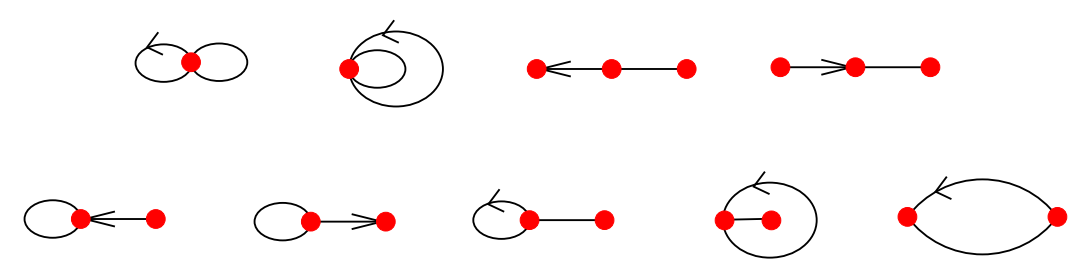

Figure 4: The rooted planar maps with two edges

(a) [3] Show that the number of rooted planar maps with $n$ edges is equal to

$$
\frac{2(2 n) ! 3^{n}}{n !(n+1) !}=\frac{2 \cdot 3^{n}}{n+2} C_{n}
$$

(b) $[2+]$ Show that the total number of vertices of all rooted planar maps with $n$ edges is equal to $3^{n} C_{n}$.

(c) [3-] Show that the number of pairs $(G, T)$, where $G$ is a rooted planar map with $n$ edges and $T$ is a spanning tree of $G$, is equal to $C_{n} C_{n+1}$.

6.C4 A $k$-triangulation of a convex $n$-gon $C$ is a maximal collection of diagonals such that there are no $k+1$ of these diagonals for which any two intersect in their interiors. A 1-triangulation is just an ordinary triangulation, enumerated by the Catalan number $C_{n-2}$ (Corollary 6.2.3(vi)). Note that any $k$-triangulation contains all diagonals between vertices at most distance $k$ apart (where the distance between two vertices $u, v$ is the least number of edges of $C$ we need to traverse in walking from $u$ to $v$ along the boundary of $C$ ). We call these $n k$ edges superfluous. For example, there are three 2-triangulations of a hexagon, illustrated below (nonsuperfluous edges only).
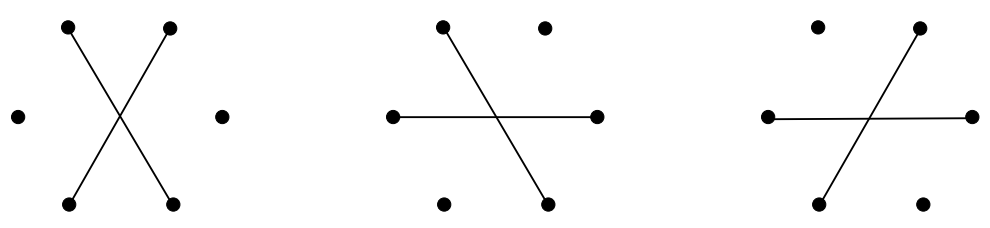

(a) [3-] Show that all $k$-triangulations of an $n$-gon have $k(n-2 k-1)$ nonsuperfluous edges. 
(b) [3] Show that the number $T_{k}(n)$ of $k$-triangulations of an $n$-gon is given by

$$
\begin{aligned}
T_{k}(n) & =\operatorname{det}\left[C_{n-i-j}\right]_{i, j=1}^{k} \\
& =\prod_{1 \leq i<j \leq n-2 k} \frac{2 k+i+j-1}{i+j-1},
\end{aligned}
$$

the latter equality by Theorem 2.7.1 and Exercise 7.101(a).

(c) $[3+]$ It follows from (b) and Exercise 7.101(a) that $T_{k}(n)$ is equal to the number of plane partitions, allowing 0 as a part, of the staircase shape $\delta_{n-2 k}=(n-2 k-1, n-2 k-2, \ldots, 1)$ and largest part at most $k$. Give a bijective proof.

6.C5 [3- $]$ Let $D$ be a Dyck path with $2 n$ steps, and let $k_{i}(D)$ denote the number of up steps in $D$ from level $i-1$ to level $i$. Show that

$$
\sum_{D, D^{\prime}} \sum_{i} k_{i}(D) k_{i}\left(D^{\prime}\right)=C_{2 n}-C_{n}^{2}
$$

where the first sum ranges over all pairs $\left(D, D^{\prime}\right)$ of Dyck paths with $2 n$ steps.

6.C6 Let $P_{k, n}$ denote the set of all subsets $S$ of $[k n]$ such that all subsets $T$ of $S$ that are maximal with respect to being a set of consecutive integers have cardinality divisible by $k$. For instance, when $n \geq 6$ and $k=3$ we could have $S=\{2,3,4,7,8,9,10,11,12,14,15,16\}$. Partially order $P_{k, n}$ by inclusion. Thus there is a bottom element $\hat{0}=\emptyset$ and top element $\hat{1}=[k n]$. Figure 5 shows $P_{2,3}$. Show that

$$
\mu(\hat{0}, \hat{1})=(-1)^{n} \frac{1}{(k-1) n+1}\left(\begin{array}{c}
k n \\
n
\end{array}\right),
$$

where $\mu$ denotes the Möbius function of $P_{k, n}$. In particular, when $k=2$ we get $\mu(\hat{0}, \hat{1})=C_{n}$.

6.C7 $[2+]$ Let $f(n)$ denote the number of plane ternary trees on the vertex set $[n]$ such that if a vertex $i$ has a child $j$ in the leftmost or center position (of the three possible positions for children), then $i<j$. Show that $f(n)=n ! C_{n}$. The four trees for the case $n=2$ are as follows: 


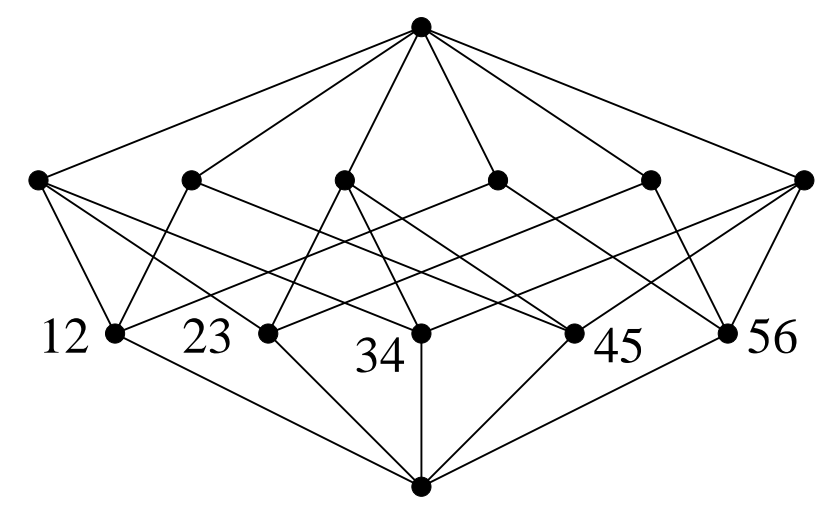

Figure 5: The poset $P_{2,3}$ of Problem C6
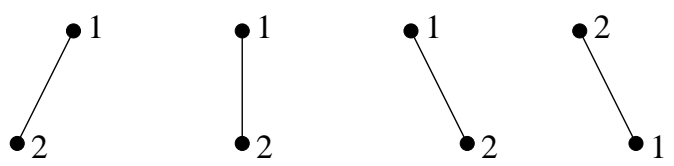

6.C8 Let $f(n)$ be the number of standard Young tableaux with exactly $2 n$ squares, at most four rows, and every row length even. Let $g(n)$ be the number of Young tableaux with exactly $2 n$ squares, exactly four rows, and every row length odd. Show that $f(n)-g(n)=C_{n}$.

6.C9 Let $t_{0}, t_{1}, \ldots$ be indeterminates. If $S$ is a finite subset of $\mathbb{N}$, then set $t^{S}=\prod_{i \in S} t_{i}$. For $X=\mathbb{N}$ or $\mathbb{P}$, let $U_{n}(X)$ denote the set of all $n$-subsets of $X$ that don't contain two consecutive integers.

(a) $[2+]$ Show that the following three power series are equal:

(i) The continued fraction

$$
\frac{1}{1-\frac{t_{0} x}{1-\frac{t_{1} x}{1-\frac{t_{2} x}{1-\cdots}}}}
$$


(ii)

$$
\frac{\sum_{n \geq 0}(-1)^{n}\left(\sum_{S \in U_{n}(\mathbb{P})} t^{S}\right) x^{n}}{\sum_{n \geq 0}(-1)^{n}\left(\sum_{S \in U_{n}(\mathbb{N})} t^{S}\right) x^{n}}
$$

(iii) $\sum_{T} \prod_{v \in V(T)} t_{\mathrm{ht}(v)}^{\operatorname{deg}(v)} x^{\# V(T)-1}$, where $T$ ranges over all (nonempty) plane trees. Moreover, $V(T)$ denotes the vertex set of $T$, ht $(v)$ the height of vertex $v$ (where the root has height 0 ), and $\operatorname{deg}(v)$ the degree (number of children) of vertex $v$.

The three power series begin

$$
1+t_{0} x+\left(t_{0}^{2}+t_{0} t_{1}\right) x^{2}+\left(t_{0}^{3}+2 t_{0}^{2} t_{1}+t_{0} t_{1}^{2}+t_{0} t_{1} t_{2}\right) x^{3}+\cdots .
$$

(b) [2] Deduce that

$$
\lim _{n \rightarrow \infty} \frac{\sum_{i \geq 0}(-1)^{i}\left(\begin{array}{c}
n-i \\
i
\end{array}\right) x^{i}}{\sum_{i \geq 0}(-1)^{i}\left(\begin{array}{c}
n+1-i \\
i
\end{array}\right) x^{i}}=\sum_{k \geq 0} C_{k} x^{k}
$$

(c) [3] Suppose that $t_{0}, t_{1}, \ldots$ are positive integers such that $t_{0}$ is odd and $\Delta^{k} t_{n}$ is divisible by $2^{k+1}$ for all $k \geq 1$ and $n \geq 0$, where $\Delta$ is the difference operator of Section 1.4. For example, $t_{i}=(2 i+1)^{2}$ satisfies these hypotheses. Let $\sum_{n \geq 0} D_{n} x^{n}$ denote the resulting power series (6). Show that $\nu_{2}\left(D_{n}\right)=\nu_{2}\left(C_{n}\right)$, where $\nu_{2}(m)$ is the exponent of the largest power of 2 dividing $m$.

6.C10 (a) $[2+]$ Start with the monomial $x_{12} x_{23} x_{34} \cdots x_{n, n+1}$, where the variables $x_{i j}$ commute. Continually apply the "reduction rule"

$$
x_{i j} x_{j k} \rightarrow x_{i k}\left(x_{i j}+x_{j k}\right)
$$

in any order until unable to do so, resulting in a polynomial $P_{n}\left(x_{i j}\right)$. Show that $P_{n}\left(x_{i j}=1\right)=C_{n}$. (Note. The polynomial $P_{n}\left(x_{i j}\right)$ itself depends on the order in which the reductions are applied.) For instance, when $n=3$ one possible sequence of 
reductions (with the pair of variables being transformed shown in boldface) is given by

$$
\begin{aligned}
\boldsymbol{x}_{\mathbf{1 2}} \boldsymbol{x}_{\mathbf{2 3}} x_{34} \rightarrow & \boldsymbol{x}_{\mathbf{1 3}} x_{12} \boldsymbol{x}_{\mathbf{3 4}}+\boldsymbol{x}_{\mathbf{1 3}} x_{23} \boldsymbol{x}_{\mathbf{3 4}} \\
\rightarrow & x_{14} x_{13} x_{12}+x_{14} x_{34} x_{12} \\
& \quad+x_{14} x_{13} x_{23}+x_{14} \boldsymbol{x}_{\mathbf{3 4}} \boldsymbol{x}_{\mathbf{2 3}} \\
\rightarrow & x_{14} x_{13} x_{12}+x_{14} x_{34} x_{12} \\
& \quad+x_{14} x_{13} x_{23}+x_{14} x_{24} x_{23}+x_{14} x_{24} x_{34} \\
= & P_{3}\left(x_{i j}\right) .
\end{aligned}
$$

(b) [3-] More strongly, replace the rule (8) with

$$
x_{i j} x_{j k} \rightarrow x_{i k}\left(x_{i j}+x_{j k}-1\right),
$$

this time ending with a polynomial $Q_{n}\left(x_{i j}\right)$. Show that

$$
Q_{n}\left(x_{i j}=\frac{1}{1-x}\right)=\frac{N(n, 1)+N(n, 2) x+\cdots+N(n, n) x^{n-1}}{(1-x)^{n}},
$$

where $N(n, k)$ is a Narayana number (defined in Exercise 6.36).

(c) [3-] Even more generally, show that

$$
Q_{n}\left(x_{i j}=t_{i}\right)=\sum(-1)^{n-k} t_{i_{1}} \cdots t_{i_{k}},
$$

where the sum ranges over all pairs $\left(\left(a_{1}, a_{2}, \ldots, a_{k}\right),\left(i_{1}, i_{2}, \ldots, i_{k}\right)\right) \in$ $\mathbb{P}^{k} \times \mathbb{P}^{k}$ satisfying $1=a_{1}<a_{2}<\cdots<a_{k} \leq n, 1 \leq i_{1} \leq i_{2} \leq$ $\cdots \leq i_{k}$, and $i_{j} \leq a_{j}$. For instance,

$$
Q_{3}\left(x_{i j}=t_{i}\right)=t_{1}^{3}+t_{1}^{2} t_{2}+t_{1}^{2} t_{3}+t_{1} t_{2}^{2}+t_{1} t_{2} t_{3}-2 t_{1}^{2}-2 t_{1} t_{2}-t_{1} t_{3}+t_{1} .
$$

(d) $[3+]$ Now start with the monomial $\prod_{1<i<j<n+1} x_{i j}$ and apply the reduction rule (8) until arriving at a polynomial $R_{n}\left(x_{i j}\right)$. Show that

$$
R_{n}\left(x_{i j}=1\right)=C_{1} C_{2} \cdots C_{n} .
$$

(e) [5-] Generalize (d) in a manner analogous to (b) and (c). 
6.C11 [3-] Let $V_{r}$ be the operator on (real) polynomials defined by

$$
V_{r}\left(\sum_{i \geq 0} a_{i} q^{i}\right)=\sum_{i \geq r} a_{i} q^{i}
$$

Define $B_{1}(q)=-1$, and for $n>1$,

$$
B_{n}(q)=(q-1) B_{n-1}(q)+V_{(n+1) / 2}\left(q^{n-1}(1-q) B_{n-1}(1 / q)\right) .
$$

Show that $B_{2 n}(1)=B_{2 n+1}(1)=(-1)^{n+1} C_{n}$.

6.C12 (a) $[3+]$ Let $g(n)$ denote the number of $n \times n \mathbb{N}$-matrices $M=\left(m_{i j}\right)$ where $m_{i j}=0$ if $j>i+1$, with row and column sum vector $\left(1,3,6, \ldots,\left(\begin{array}{c}n+1 \\ 2\end{array}\right)\right)$. For instance, when $n=2$ there are the two matrices

$$
\left[\begin{array}{ll}
1 & 0 \\
0 & 3
\end{array}\right] \quad\left[\begin{array}{ll}
0 & 1 \\
1 & 2
\end{array}\right]
$$

while an example for $n=5$ is

$$
\left[\begin{array}{ccccc}
0 & 1 & 0 & 0 & 0 \\
0 & 1 & 2 & 0 & 0 \\
1 & 0 & 3 & 2 & 0 \\
0 & 0 & 1 & 4 & 5 \\
0 & 1 & 0 & 4 & 10
\end{array}\right]
$$

Show that $g(n)=C_{1} C_{2} \cdots C_{n}$.

(b) $[2+]$ Let $f(n)$ be the number of ways to write the vector

$$
\left(1,2,3, \ldots, n,-\left(\begin{array}{c}
n+1 \\
2
\end{array}\right)\right) \in \mathbb{Z}^{n+1}
$$

as a sum of vectors $e_{i}-e_{j}, 1 \leq i<j \leq n+1$, without regard to order, where $e_{k}$ is the $k$ th unit coordinate vector in $\mathbb{Z}^{n+1}$. For instance, when $n=2$ there are the two ways $(1,2,-3)=$ $(1,0,-1)+2(0,1,-1)=(1,-1,0)+3(0,1,-1)$. Assuming (a), show that $f(n)=C_{1} C_{2} \cdots C_{n}$.

(c) [3-] Let $\mathrm{CR}_{n}$ be the convex polytope of all $n \times n$ doubly-stochastic matrices $A=\left(a_{i j}\right)$ satisfying $a_{i j}=0$ if $i>j+1$. It is easy to see that $\mathrm{CR}_{n}$ is an integral polytope of dimension $\left(\begin{array}{l}n \\ 2\end{array}\right)$. Assuming (a) 
or (b), show that the relative volume of $\mathrm{CR}_{n}$ (as defined $\S 4.6$ ) is given by

$$
\nu\left(\mathrm{CR}_{n}\right)=\frac{C_{1} C_{2} \cdots C_{n-1}}{\left(\begin{array}{l}
n \\
2
\end{array}\right) !} .
$$

6.C13 [3-] Join $4 m+2$ points on the circumference of a circle with $2 m+1$ nonintersecting chords, as in Exercise 6.19(n). Call such a set of chords a net. The circle together with the chords forms a map with $2 m+2$ (interior) regions. Color the regions red and blue so that adjacent regions receive different colors. Call the net even if an even number of regions are colored red and an even number blue, and odd otherwise. The figure below shows an odd net for $m=2$.

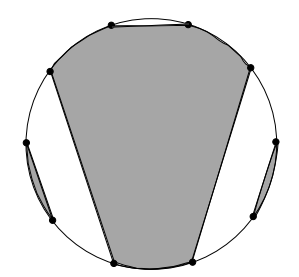

Let $f_{e}(m)$ (respectively, $f_{o}(m)$ ) denote the number of even (respectively, odd) nets on $4 m+2$ points. Show that

$$
f_{e}(m)-f_{o}(m)=(-1)^{m-1} C_{m} .
$$

6.C14 [3- $]$ Let $f(n, k)$ be the number of free (unrooted) trees $T$ with $2 n$ endpoints (leaves) and $k$ interior vertices satisfying the following properties. The interior vertices of $T$ are unlabelled. No interior vertex of $T$ has degree 2. Let $e$ be an edge of $T$, so that $T-e$ is a forest with two components. Then there is exactly one index $1 \leq i \leq n$ for which $i$ is one component and $i^{\prime}$ in the other. There is also exactly one index $1 \leq j \leq n$ for which $j$ is in one component and $(j+1)^{\prime}$ is in the other (where the index $j+1$ is taken modulo $n$ ). Figure 6 shows the four such trees with $n=3$. When $n=4$ we have $f(4,1)=1, f(4,2)=8$, $f(4,3)=12$. Show that

$$
\sum_{k=1}^{n-1}(-1)^{n-1-k} f(n, k)=C_{n} .
$$



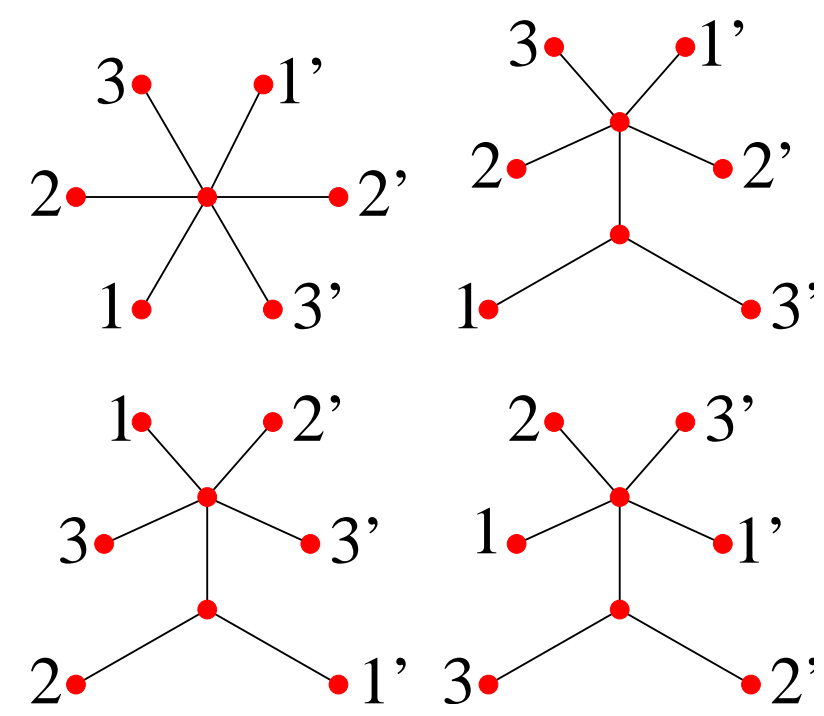

Figure 6: The trees of Exercise 6.C14 for $n=3$

6.C15 [3] Fix $n \geq 1$. Let $f(n)$ be the most number of $n$-element subsets of $\mathbb{P}$ with the following property. If $a_{1}<a_{2}<\cdots<a_{n}$ and $b_{1}<b_{2}<\cdots<$ $b_{n}$ are the elements of any two of the subsets, then there exists $i \neq j$ for which $a_{i}=b_{j}$. Show that $f(n) \neq C_{n}$ for $n \gg 0$. Note. Originally this problem was rated [5], and the problem was to show that $f(n)=C_{n}$. Since in fact $f(n) \neq C_{n}$ in general, this problem is no longer suitable for this Addendum. However, I am keeping it here for those who might have been interested in the original form of the problem.

6.C16 $[3+]$ Let $\exp \left(E \tan ^{-1}(x)\right)=\sum_{n \geq 0} c_{n}(E) x^{n}$. Define

$$
\begin{aligned}
& A_{1}(x)=\sum_{n \geq 0} c_{n}(E) \frac{x^{n}}{n !} \\
& A_{2}(x)=\sqrt{1+x^{2}} A_{1}(x) \\
& A_{3}(x)=\frac{A_{1}(x)}{\sqrt{1+x^{2}}} .
\end{aligned}
$$

For any power series $F(x)=\sum f_{n}(E) x^{n}$ let $F^{\prime}(x)=\sum_{n \geq 1} n f_{n} x^{n-1}$ and $I F(x)=\sum_{n \geq 0} f_{n} \frac{x^{n+1}}{n+1}$. For $1 \leq r \leq 3$ define $B_{r}(x)$ by writing $A_{r}(x)^{2}-A_{r}^{\prime}(x) \cdot I A_{r}(x)$ as a power series in $x$ whose coefficients are 
polynomials in $E$, and then replacing each $E^{j}$ with the Euler number $E_{j}$.

(a) Show that the coefficient of $x^{2 m-1} /(2 m-1)$ ! in $B_{1}(x)$ is $C_{m}$.

(b) Show that the coefficient of $x^{2 m} /(2 m)$ ! in $B_{2}(x)$ is $C_{m+1}$ for $m \geq 2$.

(c) Show that the coefficient of $x^{2 m} /(2 m)$ ! in $B_{3}(x)$ is $C_{m}$.

6.C17 (a) $[2+]$ Show that

$$
C(x)^{q}=\sum_{n \geq 0} \frac{q}{n+q}\left(\begin{array}{c}
2 n-1+q \\
n
\end{array}\right) x^{n} .
$$

(b) $[2+]$ Show that

$$
\frac{C(x)^{q}}{\sqrt{1-4 x}}=\sum_{n \geq 0}\left(\begin{array}{c}
2 n+q \\
n
\end{array}\right) x^{n} .
$$

6.C18 (a) [2-] Give a generating function proof of the identity

$$
\sum_{k=0}^{n} C_{2 k} C_{2(n-k)}=4^{n} C_{n}
$$

(b) [5-] Give a bijective proof.

6.C19 (a) $[2+]$ Find all power series $F(t) \in \mathbb{C} \llbracket t \rrbracket$ such that if

$$
\frac{1-x+x t F(t)}{1-x+x^{2} t}=\sum_{n \geq 0} f_{n}(x) t^{n},
$$

then $f_{n}(x) \in \mathbb{C}[x]$.

(b) $[2+]$ Find the coefficients of the polynomials $f_{n}(x)$.

6.C20 [3] Let $G$ be a finite group with identity $e$ and group $\operatorname{ring} \mathbb{Z} G$. Let $T$ be a subset of $G$, and write $\widetilde{T}=\sum_{g \in T} g \in \mathbb{Z} G$. The elements $\widetilde{T}$ are called simple quantities. Define $\widetilde{T}^{t}=\sum_{g \in T} g^{-1}$. A Schur ring over $G$ is a subring $\mathcal{S}$ of $\mathbb{Z} G$ that is generated as a $\mathbb{Z}$-module by simple quantities $\widetilde{T}_{1}, \ldots, \widetilde{T}_{r}$, called the basic sets of $\mathcal{S}$, satisfying the axioms: 
- $T_{1}=\{e\}$

- $T_{1}, \ldots, T_{r}$ form a partition of $G$,

- For each $i$ there is a $j$ such that $\widetilde{T}_{i}^{t}=\widetilde{T}_{j}$.

Let $\mathcal{S}$ be a Schur ring over $G=\mathbb{Z} / n \mathbb{Z}$. We say that $\mathcal{S}$ is decomposable if there is a nontrivial, proper subgroup $H$ of $\mathbb{Z} / n \mathbb{Z}$ such that for every basic set $T$, either $T \subseteq H$ or $T=\bigcup_{h \in T} H h$; otherwise $\mathcal{S}$ is indecomposable. Show that if $p$ is an odd prime, then the number of indecomposable Schur rings over $\mathbb{Z} / p^{m} \mathbb{Z}$ is equal to $\tau(p-1) C_{m-1}$, where $\tau(p-1)$ is the number of (positive) divisors of $p-1$.

6.C21 This exercise assumes a knowledge of matroid theory. Given a Dyck path $P=a_{1} a_{2} \cdots a_{2 n}$ of length $2 n$ (where each $a_{i}=U$ or $D$ ), let $\mathcal{U}(P)=\left\{i: a_{i}=U\right\}$, the up-step set of $P$.

(a) $[2+]$ Show that the set $\mathcal{B}_{n}$ of the up-step sets of all Dyck paths of length $2 n$ is the collection of bases of a matroid $\boldsymbol{C}_{n}$ on the set $[2 n]$, called the Catalan matroid.

(b) [2] Show that $\boldsymbol{C}_{n}$ is self-dual.

(c) $[2+]$ Let $a(P)$ denote the number of up-steps of $P$ before the first down-step, and let $b(P)$ denote the number of times the Dyck path returns to the $x$-axis after its first up-step. Show that the Tutte polynomial of $\boldsymbol{C}_{n}$ is given by

$$
T_{\boldsymbol{C}_{n}}(q, t)=\sum_{P} q^{a(P)} t^{b(P)},
$$

where the sum is over all Dyck paths $P$ of length $2 n$.

(d) $[2+]$ Show that

$$
\sum_{n \geq 0} T_{\boldsymbol{C}_{n}}(q, t) x^{n}=\frac{1+(q t-q-t) x C(x)}{1-q t x+(q t-q-t) x C(x)} .
$$

6.C22 (a) [3-] Find the unique continuous function $f(x)$ on $\mathbb{R}$ satisfying for all $n \in \mathbb{N}$ :

$$
\int_{-\infty}^{\infty} x^{n} f(x) d x=\left\{\begin{aligned}
C_{k}, & \text { if } n=2 k \\
0, & \text { if } n=2 k+1
\end{aligned}\right.
$$


(b) [3-] Find the unique continuous function $f(x)$ for $x>0$ satisfying for all $n \in \mathbb{N}$ :

$$
\int_{0}^{\infty} x^{n} f(x) d x=C_{n}
$$

6.C23 Let $f(n, k)$ be the number of 312 -avoiding permutations of $1,2, \ldots, k n$ such that $j k+1, j k+2, \ldots,(j+1) k$ appear in increasing order for $0 \leq j \leq n-1$. For instance, when $n=3$ and $k=2$ we have the 12 permutations 123456, 123546, 123564, 132456, 132546, 132564, 134256, $134526,134562,135426,135462,135642$. Show that

$$
f(n, k)=\frac{1}{k n+1}\left(\begin{array}{c}
(k+1) n \\
k+1
\end{array}\right)
$$

the number of $(k+1)$-ary trees with $n$ vertices (a Fuss-Catalan number).

6.C24 $[2+]$ The Fibonacci tree $F$ is the rooted tree with root $v$, such that the root has degree one, the child of every vertex of degree one has degree two, and the two children of every vertex of degree two have degrees one and two. Figure 7 shows the first six levels of $F$. Let $f(n)$ be the number of closed walks in $F$ of length $2 n$ beginning at $v$. Show that

$$
f(n)=\frac{1}{2 n+1}\left(\begin{array}{c}
3 n \\
n
\end{array}\right)
$$

the number of ternary trees with $n$ vertices. (See Exercises 5.45, 5.46, and 5.47(b) for further occurences of this number.) 


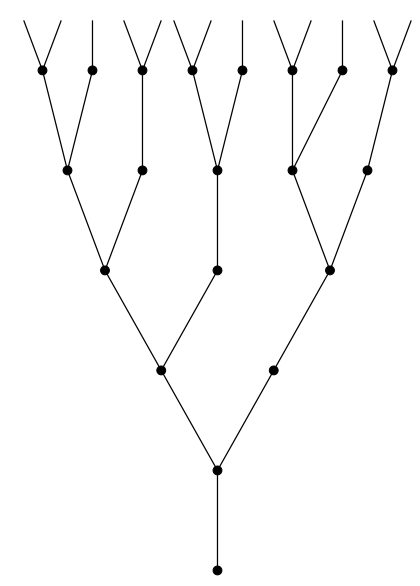

Figure 7: The Fibonacci tree

\section{SOLUTIONS}

6.19(ooo) Traverse the tree in preorder. When going down an edge (i.e., away from the root) record 1 if this edge goes to the left or straight down, and record -1 if this edge goes to the right. This gives a bijection with $\left(d^{6}\right)$.

(ppp) The proof follows from the generating function identity

$$
\begin{aligned}
C(x)^{2} & =\frac{C(x)-1}{x} \\
& =\sum_{k \geq 0} x^{k} \sum_{i=0}^{k} O(x)^{i} 2^{k-i} E(x)^{k-i} \\
& =\frac{1}{(1-2 x E(x))(1-x O(x))} .
\end{aligned}
$$

This result is due to L. Shapiro, private communication dated 26 December 2001, who raises the question of giving a simple bijective proof. In a preprint entitled "Catalan trigonometry" he gives a simple bijective proof of the related identity

$$
O(x)=x\left(O(x)^{2}+E(x)^{2}\right)
$$

and remarks that there is a similar proof of

$$
E(x)=1+2 x E(x) O(x) .
$$


For a further identity of this nature, see Exercise 6.C18.

(qqq) Given a tree $T$ of the type being counted, start with an up step $U$. Continue with the usual preorder bijection between plane trees and Dyck paths (Corollary 6.2.3), except that at a blue leaf take the steps $D U$ rather than $U D$. Finish with a down step $D$. This gives a bijection with the Dyck paths of (i). This result is due to L. Shapiro, private communication dated 24 May 2002.

(rrr) Traverse the tree in preorder. Replace an edge that is traversed for the first time (i.e., away from the root) by $1,-1$ if its vertex $v$ furthest from the root has no siblings, by 1 if $v$ is a left child, and by -1 if $v$ is a right child, yielding a bijection with the ballot sequences of (r). This item is due to E. Deutsch, private communication dated 27 February 2007. Note the similarity to $\left(\mathrm{l}^{5}\right),\left(\mathrm{p}^{4}\right)$, and $\left(\mathrm{g}^{4}\right)$.

(sss) Let $B(x)=x C(x)$, so $B(x)=x+B(x)^{2}$. Hence $B(x)=x+$ $x B(x)+B(x)^{3}$, from which the proof follows. We can also give a recursively defined bijection between the binary trees of (c) and the trees being counted, basically by decomposing a binary tree into three subtrees: the left subtree of the root, the left subtree of the right subtree of the root, and the right subtree of the right subtree of the root. This item is due to I. Gessel (private communication dated 15 May 2008).

(ttt) Take $n$ nonintersecting chords joining $2 n$ points $1,2, \ldots, 2 n$ on the circumference of a circle as in (n). Let $T$ be the "interior dual graph" of these chords together with additional edges between every two consecutive vertices, as illustrated in Figure 8. Thus $T$ is an unrooted plane tree with $n+1$ vertices. Let the root edge $e$ of $T$ be the edge crossing the chord contain vertex 1 . Let $u, v$ be the vertices of $e$, and color $u$ red (respectively, blue) if in walking from $u$ to $v$ vertex 1 is on the left (respectively, right). This procedure sets up a bijection between (n) and the present item.

The result of this item is due to M. Bóna, M. Bousquet, G. Labelle, and P. Leroux, Adv. Appl. Math. 24 (2000), 22-56 (the case $m=2$ of Theorem 10). A bijection with the noncrossing partitions of (pp) similar to the bijection above was later given by D. Callan and L. Smiley, Noncrossing partitions under rotation and reflection, preprint; math.CO/0510447. 


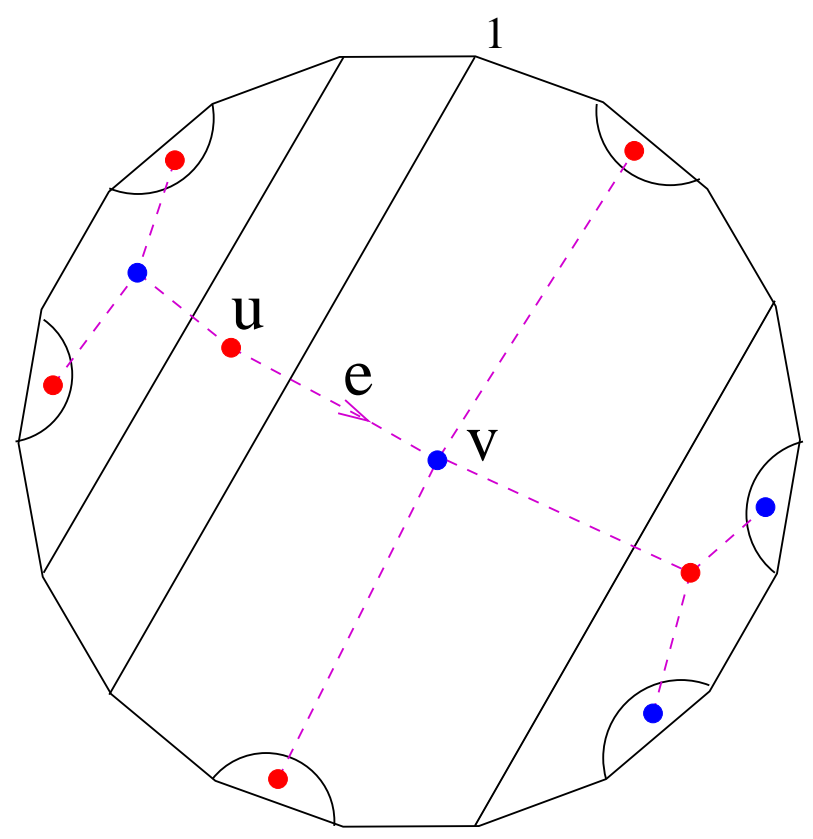

Figure 8: A bicolored edge-rooted plane tree associated with nonintersecting chords 


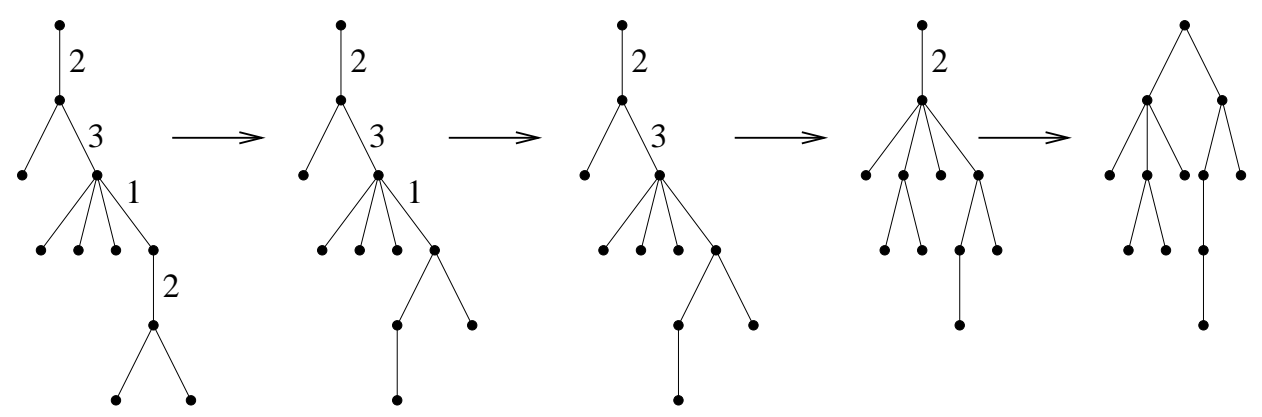

Figure 9: An example of the bijection of item (uuu)

(uuu) We obtain a bijection with plane trees (item (e)) as follows. First, erase the leaf labels and transfer the remaining labels from a vertex to the parent edge. Then process the labelled edges from bottom to top: for an edge with label $i$, transfer the $i-1$ rightmost subtrees of the child vertex, preserving order, to become the rightmost subtrees of the parent vertex, and then erase the label. See Figure 9 for an example.

Trees satisfying conditions (i) and (ii) are known as $\beta(1,0)$-trees, due to Claesson, Kitaev, and Steingrimsson, arXiv:0801.4037. The additional condition (iii) and bijection above are due to David Callan (private communication dated 19 February 2008).

(vvv) Associate with a tree $T$ being counted a sequence $\left(a_{1}, \ldots, a_{n}\right)$, where $a_{i}=\operatorname{outdeg}(i)-1$. This sets up a bijection with (w) (D. Callan, private communication dated 28 May 2010).

(www) A bijection with item $\mathrm{h}^{5}$ was given by Jang Soo Kim, SIAM J. Discrete Math. 25(1) (2011), 447-461. See also S. Forcey, M. Kafashan, M. Maliki, and M. Strayer, Recursive bijections for Catalan objects, arXiv:1212.1188.

(xxx) See the numbers $U_{m}$ of S. J. Cyvin, J. Brunvoll, E. Brendsdal, B. N. Cyvin, and E. K. Lloyd, J. Chem. Inf. Comput. Sci. 35 (1995), $743-751$.

(yyy) The labelled trees being counted are just the plane trees of (e) with the preorder (or depth-first) labeling. A more complicated bijection with Dyck paths appears in Section 2.3 of the paper A. Asinowski and T. Mansour, Europ. J. Combinatorics 29 (2008), $1262-1279$. 
(zzz) List the non-endpoint vertices $v_{1}, \ldots, v_{r}$ in increasing order along with their degrees (number of children) $d_{1}, \ldots, d_{r}$. Then the pairs $\left(\left(d_{1}, d_{2}, \ldots, d_{r}\right),\left(v_{2}-v_{1}, v_{3}-v_{2}, \ldots, v_{r}-v_{r-1}, n+1-v_{r}\right)\right)$ are in bijection with $\left(\mathrm{s}^{6}\right)$. For instance, the five trees listed in the statement of this item correspond to the pairs $(3,3),(21,12),(21,21)$, $(12,12),(111,111)$ in that order. This item is due to David Callan, private communication dated 1 September 2007. Note the noncrossing/nonnesting analogy between the pairs of items $((p),(q))$, $((\mathrm{pp}),(\mathrm{uu})),\left((\mathrm{o}),\left(\mathrm{e}^{5}\right)\right)$, and ((yyy),(zzz)).

$\left(\mathrm{a}^{4}\right)$ Add one further up step and then down steps until reaching $(2 n, 0)$. This gives a bijection with the Dyck paths of (i).

$\left(b^{4}\right)$ Deleting the first UD gives a bijection with (i) (Dyck paths of length $2 n$ ). This result is due to David Callan, private communication dated 3 November 2004.

$\left(c^{4}\right)$ The generating function for Dyck paths with no peaks at the $x$ axis (or hill-free Dyck paths) is

$$
F(x)=\frac{1}{1-x^{2} C(x)^{2}} .
$$

The generating function for hill-free Dyck paths with leftmost peak at height 2 is $x^{2} C(x) F(x)$. The generating function for hillfree Dyck paths with leftmost peak at height 3 is $x^{3} C(x)^{2} F(x)$. But it can be checked that

$$
x^{2} C(x) F(x)+x^{3} C(x)^{2} F(x)=x(C(x)-1),
$$

and the proof follows. This result is due to E. Deutsch, private communication dated 30 December 2006. Is there a nice bijective proof?

$\left(d^{4}\right)$ See D. Callan, math.C0/0511010.

$\left(\mathrm{e}^{4}\right)$ Given such a path, delete the first and last steps and every valley (downstep immediately followed by an upstep). This is a bijection to Dyck paths of length $2 n$ (item (i)) because the paths being counted necessarily have $n-1$ valleys. Another nice bijection is the following. Traverse the plane binary trees of (d) in preorder. To each node of degree $r$ associate $r$ up steps followed by 1 down 
step, except that the last leaf is ignored. We then get a Dyck path such that every ascent has length 2, clearly in bijection with those for which every descent has length 2 .

This item is due independently to D. Callan (first proof above, private communication dated 1 September 2007) and E. Deutsch (second proof, private communication dated 6 September 2007).

$\left(\mathrm{f}^{4}\right)$ In the two-colored Motzkin paths of $\left(\mathrm{n}^{4}\right)$ replace the step $(1,1)$ with the sequence of steps $(1,1)+(1,1)+(1,-1)$, the step $(1,-1)$ with $(1,1)+(1,-1)+(1,-1)$, the red step $(1,0)$ with $(1,1)+$ $(1,-1)$, and the blue step $(1,0)$ with $(1,1)+(1,1)+(1,-1)+$ $(1,-1)$.

$\left(\mathrm{g}^{4}\right)$ Traverse the trees of (rrr) in preorder. Replace a vertex of degree $d$ encountered for the first time with $1^{d},-1$ (i.e., $d$ 1's followed by -1 ), except do nothing for the last leaf. This gives a bijection with the present item. This item is due to E. Deutsch, private communication dated 27 February 2007. Note the similarity to $\left(1^{5}\right),\left(\mathrm{p}^{4}\right)$, and (rrr).

$\left(h^{4}\right)$ For each Dyck path of length $2 n$ with at least one valley (i.e., an occurrence of DU), insert a UD after the first DU. For the unique Dyck path of length $2 n$ without a valley (viz., $U^{n} D^{n}$ ), insert $\mathrm{U}$ at the beginning and $\mathrm{D}$ at the end. This sets up a bijection with (i). This result is due to E. Deutsch, private communication dated 19 November 2005.

$\left(^{4}\right)$ Every Dyck path $P$ with at least two steps has a unique factorization $P=X Y Z$ such that $Y$ is a Dyck path (possibly with 0 steps), length $(X)=\operatorname{length}(Z)$, and $X Z$ is a Dyck path (with at least two steps) of the type being counted. Hence if $f(n)$ is the number of Dyck paths being counted and $F(x)=\sum_{n \geq 1} f(n-1) x^{n}$, then

$$
C(x)=1+F(x) C(x) .
$$

It follows that $F(x)=x C(x)$, so $f(n)=C_{n}$ as desired. This result is due to Sergi Elizalde (private communication, September, 2002).

$\left(j^{4}\right)$ To obtain a bijection with the Dyck paths of (i) add a $(1,1)$ step immediately following a path point $(m, 0)$ and a $(1,-1)$ step at the end of the path (R. Sulanke, private communication from E. Deutsch dated 4 February 2002). 
$\left(\mathrm{k}^{4}\right)$ To obtain a bijection with $\left(\mathrm{j}^{4}\right)$ contract a region under a peak of height one to a point (E. Deutsch and R. Sulanke, private communication from E. Deutsch dated 4 February 2002). Deutsch points out (private communication dated 15 October 2008) that this item and the previous are special cases of the following result. Fix a Dyck path $D$ of length $2 p \leq 2 n$. Then the number of occurrences of $D$ beginning at height 0 among all Dyck paths of length $2 n$ is $C_{n+1-p}$. There is a straightforward proof using generating functions.

$\left(1^{4}\right)$ These decompositions are equivalent to the centered tunnels of S. Elizalde, Proc. FPSAC 2003, arXiv:math.C0/0212221. Elizalde and E. Deutsch, Ann. Combinatorics 7 (2003), 281-297, give a (length-preserving) bijection from Dyck paths to themselves that take centered tunnels into hills. Now use $\left(\mathrm{k}^{4}\right)$.

$\left(\mathrm{m}^{4}\right)$ First solution. Fix a permutation $u \in \mathfrak{S}_{3}$, and let $T(u)$ be the set of all $u$-avoiding permutations (as defined in Exercise 6.39(l)) in all $\mathfrak{S}_{n}$ for $n \geq 1$. Partially order $T(u)$ by setting $v \leq w$ if $v$ is a subsequence of $w$ (when $v$ and $w$ are written as words). One checks that $T(u)$ is isomorphic to the tree $T$. Moreover, the vertices in $T(u)$ of height $n$ consist of the $u$-avoiding permutations in $\mathfrak{S}_{n+1}$. The proof then follows from Exercise 6.19(ee,ff).

Second solution. Label each vertex by its degree. A saturated chain from the root to a vertex at level $n-1$ is thus labelled by a sequence $\left(b_{1}, b_{2}, \ldots, b_{n}\right)$. Set $a_{i}=i+2-b_{i}$. This sets up a bijection between level $n-1$ and the sequences $\left(a_{1}, \ldots, a_{n}\right)$ of $(\mathrm{s})$. Third solution. Let $f_{n}(x)=\sum_{v} x^{\operatorname{deg}(v)}$, summed over all vertices of $T$ of height $n-1$. Thus $f_{1}(x)=x^{2}, f_{2}(x)=x^{2}+x^{3}, f_{3}(x)=$ $x^{4}+2 x^{3}+2 x^{2}$, etc. Set $f_{0}(x)=x$. The definition of $T$ implies that we get $f_{n+1}(x)$ from $f_{n}(x)$ by substituting $x^{2}+x^{3}+\cdots+x^{k+1}=$ $x^{2}\left(1-x^{k}\right) /(1-x)$ for $x^{k}$. Thus

$$
f_{n+1}(x)=\frac{x^{2}\left(f_{n}(1)-f_{n}(x)\right)}{1-x}, n \geq 0 .
$$

Setting $F(x, t)=\sum_{n \geq 0} F_{n}(x) t^{n}$, there follows

$$
\frac{x-F(x, t)}{t}=\frac{x^{2}}{1-x}(F(1, t)-F(x, t)) .
$$


Hence

$$
F(x, t)=\frac{x-x^{2}+x^{2} t F(1, t)}{1-x+x^{2} t} .
$$

Now use Exercise 6.C19.

The trees $T(u)$ were first defined by J. West, Discrete Math. 146 (1995), 247-262 (see also Discrete Math. 157 (1996), 363-374) as in the first solution above, and are called generating trees. West then presented the labeling argument of the second solution, thereby giving new proofs of (ee) and (ff). For further information on generating trees, see C. Banderier, M. Bousquet-Mélou, A. Denise, P. Flajolet, D. Gardy, and D. Gouyou-Beauchamps, Discrete Math. 246 (2002), 29-55.

$\left(\mathrm{n}^{4}\right)$ Replace a step $(1,1)$ with 1,1 , a step $(1,-1)$ with $-1,-1$, a red step $(1,0)$ with $1,-1$, a blue step $(1,0)$ with $-1,1$, and adjoin an extra 1 at the beginning and -1 at the end. This gives a bijection with (r) (suggested by R. Chapman). The paths being enumerated are called two-colored Motzkin paths. See for instance E. Barcucci, A. Del Lungo, E. Pergola, and R. Pinzani, Lecture Notes in Comput. Sci. 959, Springer, Berlin, 1995, pp. 254-263.

$\left(\mathrm{o}^{4}\right)$ Replace a step $(1,1)$ with 1,1 , a step $(1,-1)$ with $-1,-1$, a red step $(1,0)$ with $-1,1$, and a blue step $(1,0)$ with $1,-1$, to get a bijection with (r). This item is due to E. Deutsch, private communication dated 28 December 2006.

$\left(\mathrm{p}^{4}\right)$ Replace each level step in such a path with $1,-1$, each up step with 1 , and each down step with -1 to obtain a bijection with the ballot sequences of (r). This item is due to E. Deutsch, private communication dated 27 February 2007. Note the similarity to $\left(1^{5}\right),(\operatorname{rrr})$, and $\left(\mathrm{g}^{4}\right)$.

$\left(\mathrm{q}^{4}\right)$ Let $\pi$ be a noncrossing partition of $[n]$. Denote the steps in a Motzkin path by $U$ (up), $D$ (down) and $L$ (level). In the sequence $1,2, \ldots, n$, replace the smallest element of a nonsingleton block of $\pi$ with the two steps $L U$. Replace the largest element of a nonsingleton block of $\pi$ with $D L$. Replace the element of a singleton block with $L L$. Replace an element that is neither the smallest nor largest element of its block with $D U$. Remove the first and last terms (which are always $L$ ). For instance, if $\pi=145-26-3$, 
then we obtain $U L U L L D U D L D$. This sets up a bijection with (pp). E. Deutsch (private communication dated 16 September 2004) has also given a simple bijection with the Dyck paths (i). The bijection given here is a special case of a bijection appearing in W. Chen, E. Deng, R. Du, R. Stanley, and C. Yan, Trans. Amer. Math. Soc. 359 (2007), 1555-1575.

$\left(\mathrm{r}^{4}\right)$ This result is equivalent to the identity

$$
C_{n}=\sum_{k=0}^{n-1}\left(\begin{array}{c}
n-1 \\
k
\end{array}\right) M_{k}
$$

which can be proved in various ways. See the paragraph preceding Corollary 5.5 of A. Claesson and S. Linusson, Proc. Amer. Math. Soc. 139 (2011), 435-449; arXiv: 1003.4728.

$\left(s^{4}\right)$ Replace each level step with an up step followed by a down step to obtain a bijection with the Dyck paths (i). This item and the two following are due to L. Shapiro, private communication dated 19 August 2005.

$\left(\mathrm{t}^{4}\right)$ Add an up step at the beginning and a down step at the end. Then replace each level step at height one with a down step followed by an up step, and replace all other level steps with an up step followed by a down step. We obtain a bijection with Dyck paths.

$\left(\mathrm{u}^{4}\right)$ Exactly the same rule as in the solution to $\left(\mathrm{s}^{4}\right)$ gives a bijection with Dyck paths.

$\left(v^{4}\right)$ Replace each level step with a down step followed by an up step, prepend an up step, and append a down step to obtain a bijection with Dyck paths (i) (E. Deutsch, private communication dated 20 December 2006).

$\left(\mathrm{w}^{4}\right)$ Let $U, D, H$ denote an up step, a down step, and a horizontal (level) step, respectively. Every nonempty Schröder path has uniquely the form $H A$ or $U B D C$, where $A, B, C$ are Schröder paths. Define recursively $\emptyset^{\prime}=\emptyset,(H A)^{\prime}=H A^{\prime},(U B D C)^{\prime}=$ $U C^{\prime} D B^{\prime}$. This defines an involution on Schröder paths from $(0,0)$ to $(2 n-2,0)$ that interchanges valleys $D U$ and double rises $U U$. Hence it provides a bijection between the current item and $\left(\mathrm{v}^{4}\right)$ (E. Deutsch, private communication dated 20 December 2006). 
Deutsch points out that this involution is an unpublished generalization of his note in Discrete Math. 204 (1999), 163-166.

$\left(\mathrm{x}^{4}\right)$ Let $G=G(x)$ be the (ordinary) generating function for Schröder paths from $(0,0)$ to $(2 n, 0)$ with no double rises. Hence from $\left(\mathrm{w}^{4}\right)$ we have $G(x)=(C(x)-1) / x$. Now every Schröder path with no level steps on the $x$-axis and no double rises is either empty or has the form (using the notation of the previous item) $U A D B$; here either $A=\emptyset$ or $A=H B$, where $B$ is a Schröder path with no double rises. Hence if $F(x)$ is the generating function for Schröder paths from $(0,0)$ to $(2 n, 0)$ with no level steps on the $x$-axis and no double rises, then

$$
F(x)=1+x(1+x G(x)) F(x) .
$$

It follows easily that $F(x)=C(x)$ (E. Deutsch, private communication dated 20 December 2006). Is there a simple bijective proof?

$\left(\mathrm{y}^{4}\right)$ There is an obvious bijection with the two-colored Motzkin paths of $\left(\mathrm{n}^{4}\right)$ : replace the step $(1,1)$ with $1,(1,-1)$ with -1 , red $(1,0)$ with 0 , and blue $(1,0)$ with $0^{*}$. This item is due to E. Deutsch (private communication, 20 January 2007). Deutsch also notes a linear algebraic interpretation: let $A=\left(A_{i j}\right)_{i, j \geq 1}$ be the tridiagonal matrix with $A_{i i}=2$ and $A_{i, i+1}=A_{i, i-1}=1$. Then $C_{n}=\left(A^{n-1}\right)_{11}$.

$\left(\mathrm{z}^{4}\right)$ Replace each step $(1,1)$ or $(0,1)$ with the step $(1,1)$, and replace each step $(1,0)$ with $(1,-1)$. We obtain a bijection with the paths of $\left(a^{4}\right)$.

$\left(a^{5}\right)$ Given such a path, prepend an up step $U$ and append a down step $D=$. Each maximal segment below ground level in the elevated path has the form $D^{k} U^{k}$ for some $k \geq 1$. Replace it with $(U D)^{k}$. This gives a bijection with the Dyck paths of (i). Communication from David Callan dated 13 June 2012.

$\left(b^{5}\right)$ The bijection with Dyck paths has the same description as in $\left(a^{5}\right)$, that is, given such a path, each maximal segment below ground level has the form $D^{k} U^{k}$ for some $k \geq 1$. Replace it with $(U D)^{k}$.

$\left(c^{5}\right)$ See Section 3 of D. Callan, A combinatorial interpretation of $\frac{j}{n}\left(\begin{array}{c}k n \\ n+j\end{array}\right)$, math.CO/0604471. 
$\left(\mathrm{d}^{5}\right)$ See R. K. Guy, J. Integer Sequences 3 (2000), article 00.1.6, and R. K. Guy, C. Krattenthaler, and B. Sagan, Ars Combinatorica 34 (1992), 3-15.

$\left(\mathrm{e}^{5}\right)$ Replace the left-hand endpoint of each arc with a 1 and the righthand endpoint with a -1 . We claim that this gives a bijection with the ballot sequences of $(r)$. First note that if we do the same construction for the noncrossing matchings of (o), then it is very easy to see that we get a bijection with (r). Hence we will give a bijection from $(\mathrm{o})$ to $\left(\mathrm{e}^{5}\right)$ with the additional property that the locations of the left endpoints and right endpoints of the arcs are preserved. (Of course any bijection between (o) and $\left(\mathrm{e}^{5}\right)$ would suffice to prove the present item; we are showing a stronger result.)

Let $M$ be a noncrossing matching on $2 n$ points. Suppose we are given the set $S$ of left endpoints of the arcs of $M$. We can recover $M$ by scanning the elements of $S$ from right-to-left, and attaching each element $i$ to the leftmost available point to its right. In other words, draw an arc from $i$ to the first point to the right of $i$ that does not belong to $S$ and to which no arc has been already attached. If we change this algorithm by attaching each element of $S$ to the rightmost available point to its right, then it can be checked that we obtain a nonnesting matching and that we have defined a bijection from (o) to $\left(\mathrm{e}^{5}\right)$.

I cannot recall to whom this argument is due. Can any reader provide this information? For further information on crossings and nestings of matchings, see W. Chen, E. Deng, R. Du, R. Stanley, and C. Yan, Trans. Amer. Math. Soc. 359 (2007), 1555-1575, and the references given there.

$\left(\mathrm{f}^{5}\right)$ Let $f: \mathbb{P} \rightarrow \mathbb{P}$ be any function satisfying $f(i) \leq i$. Given a ballot sequence $\alpha=\left(a_{1}, \ldots, a_{2 n}\right)$ as in (r), define the corresponding $f$ matching $M_{\alpha}$ as follows. Scan the 1's in $\alpha$ from right-to-left. Initially all the 1's and -1 's in $\alpha$ are unpaired. When we encounter $a_{i}=1$ in $\alpha$, let $j$ be the number of unpaired -1 's to its right, and draw an arc from $a_{i}$ to the $f(j)$ th -1 to its right (thus pairing $a_{i}$ with this -1$)$. Continue until we have paired $a_{1}$, after which all terms of $\alpha$ will be paired, thus yielding the matching $M_{\alpha}$. By construction, the number of $f$-matchings of $[2 n]$ is $C_{n}$. This gives infinitely many combinatorial interpretations of $C_{n}$, but of course 


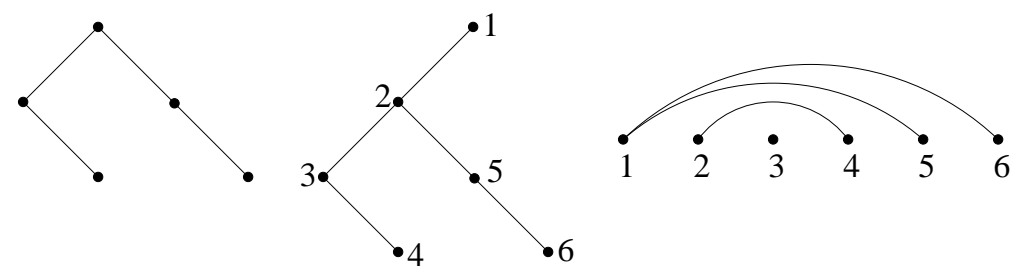

Figure 10: The bijection for Exercise 6.19(i $\left.{ }^{5}\right)$

most of these will be of no special interest. If $f(i)=1$ for all $i$, then we obtain the noncrossing matchings of (o). If $f(i)=i$ for all $i$, then we obtain the nonnesting matchings of $\left(\mathrm{e}^{5}\right)$. If $f(i)=\lfloor i / 2\rfloor$ for all $i$, then we obtain the matchings of the present item. Thus these matchings are in a sense "halfway between" noncrossing and nonnesting matchings.

$\left(g^{5}\right)$ Reading the the points from left-to-right, replace each isolated point and each point which is the left endpoint of an arc with 1 , and replace each point which is the right endpoint of an arc with -1 . We obtain a bijection with $\left(\mathrm{d}^{6}\right)$.

$\left(\mathrm{h}^{5}\right)$ Label the points $1,2, \ldots, n$ from left-to-right. Given a noncrossing partition of $[n]$ as in (pp), draw an arc from the first element of each block to the other elements of that block, yielding a bijection with the current item. This result is related to research on network testing done by Nate Kube (private communication from Frank Ruskey dated 9 November 2004).

$\left(i^{5}\right)$ Given a binary tree with $n$ vertices as in (c), add a new root with a left edge connected to the old root. Label the $n+1$ vertices by $1,2, \ldots, n+1$ in preorder. For each right edge, draw an arc from its bottom vertex to the top vertex of the first left edge encountered on the path to the root. An example is shown in Figure 10. On the left is a binary tree with $n=5$ vertices; in the middle is the augmented tree with $n+1$ vertices with the preorder labeling; and on the right is the corresponding set of arcs. This result is due to David Callan, private communication, 23 March 2004.

$\left(j^{5}\right)$ If we label the vertices $1, \ldots, n+1$ from right-to-left then we obtain a lopsided representation of the trees of (vvv) (D. Callan, private communication dated 28 May 2010). 
$\left(\mathrm{k}^{5}\right)$ Replace the left-hand endpoint of each arc by $U$ (an up step), and replace the right-hand endpoint by $D$ (a down step). Replace each isolated point in even position by $U$, and in odd position by $D$. Prepend $U$ and append $D$ to get a bijection with the Dyck paths of (i). This item is due to D. Callan, private communications dated 13 September and 14 September 2007.

$\left(1^{5}\right)$ Read the vertices from left to right. Replace an isolated vertex by $1,-1$, the left endpoint of an arc by 1 , and the right endpoint of an arc with -1 to obtain a bijection with the ballot sequences of (r). This item is due to E. Deutsch, private communication dated 27 February 2007. Note the similarity to $\left(\mathrm{p}^{4}\right)$, ( $\left.\mathrm{rrr}\right)$, and $\left(\mathrm{g}^{4}\right)$. D. Bevan pointed out (private communication dated 25 August 2010) that if we replace each isolated vertex with two adjacent vertices connected by an arc, then we obtain a bijection with (o).

$\left(\mathrm{m}^{5}\right)$ See S. Elizalde, Statistics on Pattern-Avoiding Permutations, Ph.D. thesis, M.I.T., June 2004 (Proposition 3.5.3(1)).

$\left(\mathrm{n}^{5}\right)$ Three proofs are given by H. Niederhausen, Electr. J. Comb. 9 (2002), \#R33.

$\left(\mathrm{o}^{5}\right)$ Let $P$ be a parallelogram polyomino of the type being counted. Linearly order the maximal vertical line segments on the boundary of $P$ according to the level of their bottommost step. Replace such a line segment appearing on the right-hand (respectively, left-hand) path of the boundary of $P$ by a 1 (respectively, -1 ), but omit the final line segment (which will always be on the left). For instance, for the first parallelogram polyomino shown in the statement of the problem, we get the sequence $(1,1,-1,-1,1,-1)$. This sets up a bijection with (r). This result is due to E. Deutsch, S. Elizalde, and A. Reifegerste (private communication, April, 2003).

$\left(\mathrm{p}^{5}\right)$ If we consider the number of chord diagrams (n) containing a fixed horizontal chord, then we obtain the standard quadratic recurrence for Catalan numbers. An elegant "bijectivization" of this argument is the following. Fix a vertex $v$. Given a nonintersecting chord diagram with a distinguished horizontal chord $K$, rotate the chords so that the left-hand endpoint of $K$ is $v$. This gives a bijection with (n). Another way to say this (suggested by R. Chapman) 
is that there are $n$ different chord slopes, each occuring the same number of times, and hence $C_{n}$ times.

$\left(q^{5}\right)$ Kepler towers were created by X. Viennot, who gave a bijection with the Dyck paths (i). Viennot's bijection was written up by D. Knuth, Three Catalan bijections, Institut Mittag-Leffler preprint series, 2005 spring, \#04;

Www.ml.kva.se/preprints/0405s.

The portion of this paper devoted to Kepler walls is also available at

www-cs-faculty. stanford.edu/ knuth/programs/viennot.w.

$\left(\mathrm{r}^{5}\right)$ Immediate from the generating function identity

$$
C(x)=\frac{1}{1-x C(x)}=1+x C(x)+x^{2} C(x)^{2}+\cdots .
$$

This result is due to E. Deutsch (private communication dated 8 April 2005).

$\left(\mathrm{s}^{5}\right)$ Add 1 to the terms of the sequences of $(\mathrm{w})$. Alternatively, if $\left(b_{1}, \ldots, b_{n-1}\right)$ is a sequence of $(\mathrm{s})$, then let $\left(a_{1}, \ldots, a_{n}\right)=(n+1-$ $\left.b_{n-1}, b_{n-1}-b_{n-2}, \ldots, b_{2}-b_{1}\right)$.

$\left(\mathrm{t}^{5}\right)$ Let $L$ be the lattice path from $(0,0)$ to $(n, n)$ with steps $(0,1)$ and $(1,0)$ whose successive horizontal steps are at heights $a_{1}, a_{2}, \ldots, a_{n}$. (In particular, the first step must be $(0,1)$.) There will be exactly one horizontal step ending at a point $(i, i)$. Reflect the portion of $L$ from $(0,0)$ to $(i, i)$ about the line $y=x$. We obtain a bijection with the lattice paths counted by $(\mathrm{h})$. This result is due to $\mathrm{W}$. Moreira (private communication from M. Aguiar, October, 2005).

$\left(u^{5}\right)$ The positions of each $i$ that occurs form the blocks of the noncrossing partitions of item (pp). Equivalently, the sequences of this item are the "restricted growth functions" of Exercise 1.28 (= Exercise 1.106 in the second edition). See also M. Klazar, Europ. J. Combinatorics 17 (1996), 53-68. This item was suggested by D. Callan, private communication dated 16 January 2011. 
$\left(\mathrm{v}^{5}\right)$ Obvious bijection with $(\mathrm{t})$. The sequences being counted are also the positions of the down steps in the Dyck paths of length $2 n$ in item (i), and the second rows of the standard Young tableaux of (ww).

$\left(\mathrm{w}^{5}\right)$ These sequences are just the complements (within the set [2n]) of those of item $\left(\mathrm{v}^{5}\right)$. They are also the positions of the up steps in the Dyck paths of length $2 n$ in item (i), and the first rows of the standard Young tableaux of (ww).

$\left(\mathrm{x}^{5}\right)$ This result was obtained in collaboration with Yangzhou $\mathrm{Hu}$ in 2011. See Y. Hu, On the number of fixed-length semiorders, in preparation. To get a bijection with the Dyck paths $D$ of item (i), let $a_{i}-1$ be the number of down-steps before the $i$ th peak, and let $b_{i}$ be the number of up-steps in $D$ before the $i$ th peak.

$\left(\mathrm{y}^{5}\right)$ Fix a root vertex $v$ of a convex $(n+2)$-gon, and label the other vertices $0,1, \ldots, n$ in clockwise order from $v$. If the vertex $i$ is connected to $v$, then set $a_{i}=1$. If in a triangle with vertices $i<$ $j<k$ we have already computed $a_{i}$ and $a_{k}$, then let $a_{j}=a_{i}+a_{k}$. This sets up a bijection with (a). This item was provided by Jan Stevens, private communication, 10 June 2009.

$\left(\mathrm{z}^{5}\right)$ Partial sums of the sequences in (r). The sequences of this exercise appear explicitly in E. P. Wigner, Ann. Math. 62 (1955), 548-564.

$\left(a^{6}\right)$ This interpretation was conjectured by M. Albert, N. Ruškuc, and V. Vatter, reported by Vatter at

http://mathoverflow. net/questions/131585.

At this website a bijection with Dyck paths was given by $\mathrm{C}$. Stump.

$\left(b^{6}\right)$ See Theorem 9 of S. Kitaev and J. Remmel, Enumerating $(\mathbf{2}+\mathbf{2})$ free posets by the number of minimal elements and other statistics, arXix:1004.3220. The authors use the term restricted ascent sequence for the sequences being enumerated. Their Theorem 9 involves collaboration with A. Claesson, M. Dukes, and H. H. Gudmundsson.

$\left(c^{6}\right)$ These sequences are the depths of the leaves of the plane binary trees of $(\mathrm{d})$, read in preorder. This item is due to F. Hivert, private communication, 12 October 2011. 
$\left(\mathrm{d}^{6}\right)$ In $\left(\mathrm{a}^{4}\right)$ replace an up step with 1 and a down step with -1 .

$\left(\mathrm{e}^{6}\right)$ Let $B=\left(b_{1}, b_{2}, \ldots, b_{2 n}\right)$ be a ballot sequence. Define a subsequence $S$ of $\left(a_{1}, a_{2}, \ldots, a_{2 n-2}\right)$ as follows. Let $a_{2 i-1}$ belong to $S$ if the $i$ th -1 in $B$ is followed by a 1 . Let $a_{2 i}$ belong to $S$ if the $(i+1)$ st 1 in $B$ is preceded by a -1 . For instance, if $B=(1,1,1,-1,1,1,-1,1,-1,-1,-1)$, then $S=\left(a_{3}, a_{5}, a_{6}, a_{10}\right)$. The correspondence $B \mapsto S$ is a bijection between (r) and the present item.

$\left(f^{6}\right)$ Suppose that the reverse sequence $b_{1} \cdots b_{2 n-2}=a_{2 n-2} \cdots a_{1}$ begins with $k-1$ 's. Remove these -1 's, and and for each $1 \leq i \leq k$ remove the rightmost $b_{j}$ for which $b_{k+1}+b_{k+2}+\cdots+b_{j}=i$. This yields a sequence of $k+1$ ballot sequences as given by (r). Place a 1 at the beginning and -1 at the end of each of these ballot sequences and concatenate, yielding a bijection with (r). This result (stated in terms of lattice paths) is due to David Callan, private communication dated 26 February 2004.

Example. Let $a_{1} \cdots a_{14}=+--+--++++-+--$ (writing + for 1 and - for -1$)$, so $b_{1} \cdots b_{14}=--+-++++--$ +--+ . Remove $b_{1}, b_{2}, b_{5}, b_{14}$, yielding the ballot sequences +- , +++--+-- , and $\emptyset$. We end up with the ballot sequence ++--++++--+--++- .

$\left(\mathrm{g}^{6}\right)$ Consider the pairs of lattice paths of (l) and the lines $L_{i}$ defined by $x+y=i, 1 \leq i \leq n$. Let $S$ denote the set of all lattice squares contained between the two paths. The line $L_{i}$ will pass through the interior of some $b_{i}$ elements of $S$. Set $a_{i}= \pm b_{i}$ as follows: (i) $a_{1}=b_{1}=1$, (ii) $a_{i} a_{i}>0$ if $b_{i} \neq b_{i-1}$, and (iii) if $b_{i}=b_{i-1}$, then $a_{i}=a_{i-1}$ if the top lattice square in $S$ that $L_{i}$ passes through lies above the top lattice square in $S$ that $L_{i-1}$ passes through, and otherwise $a_{i}=-a_{i-1}$. This sets up a bijection with (l).

$\left(\mathrm{h}^{6}\right)$ In the tree $T$ of $\left(\mathrm{m}^{4}\right)$, label the root by 0 and the two children of the root by 0 and 1 . Then label the remaining vertices recursively as follows. Suppose that the vertex $v$ has height $n$ and is labelled by $j$. Suppose also that the siblings of $v$ with labels less than $j$ are labelled $t_{1}, \ldots, t_{i}$. It follows that $v$ has $i+2$ children, which we label $t_{1}, \ldots, t_{i}, j, n$. See Figure 11 for the labelling up to height 3. As in the second solution to Exercise $6.19\left(\mathrm{~m}^{4}\right)$, a saturated 


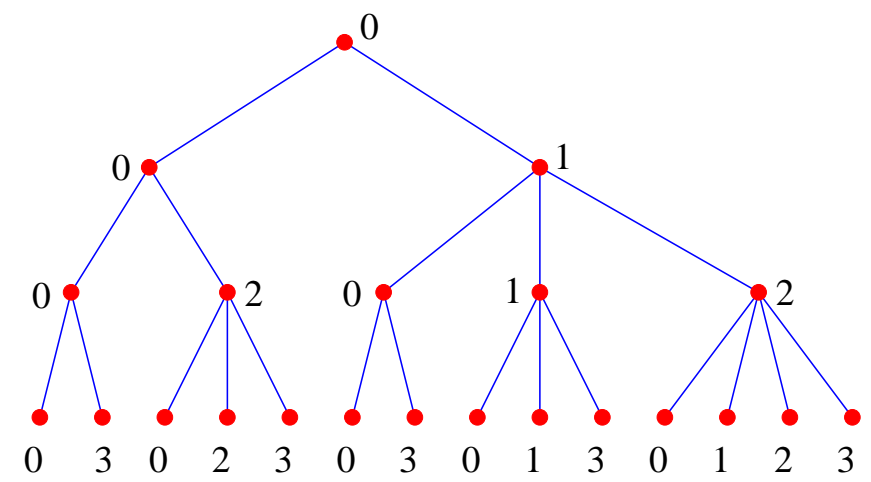

Figure 11: The tree for Exercise 6.19 $\left(\mathrm{h}^{6}\right)$

chain from the root to a vertex at level $n-1$ is thus labelled by a sequence $\left(a_{1}, a_{2}, \ldots, a_{n}\right)$. It can be seen that this sets up a bijection between level $n-1$ and the sequences we are trying to count. The proof then follows from $\left(\mathrm{m}^{4}\right)$. This exercise is due to Z. Sunik, Electr. J. Comb. 10 (2003), N5. Sunik also points out that the number of elements labelled $j$ at level $n$ is equal to $C_{j} C_{n-j}$.

$\left(i^{6}\right)$ Given a plane tree with $n$ edges, traverse the edges in preorder and record for each edge except the last the degree (number of successors) of the vertex terminating the edge. It is easy to check that this procedure sets up a bijection with (e). This result is due to David Callan, private communication dated 3 November 2004.

$\left(\mathrm{j}^{6}\right)$ Let $T$ be a plane tree with $n+1$ vertices labelled $1,2, \ldots, n+1$ in preorder. Do a depth first search through $T$ and write down the vertices in the order they are visited (including repetitions). This establishes a bijection with (e). The sequences of this exercise appear implicitly in E. P. Wigner, Ann. Math. 62 (1955), 548564 , viz., as a contribution $X_{a_{1} a_{2}} X_{a_{2} a_{3}} \cdots X_{a_{2 n-1} a_{2 n}} X_{a_{2 n} a_{1}}$ to the $(1,1)$-entry of the matrix $X^{2 n}$. Exercise $5.19\left(z^{5}\right)$ is related.

$\left(\mathrm{k}^{6}\right)$ The sequences $1,1+a_{n}, 1+a_{n}+a_{n-1}, \ldots, 1+a_{n}+a_{n-1}+\cdots+a_{2}$ coincide with those of (s). See R. Stanley, J. Combinatorial Theory 14 (1973), 209-214 (Theorem 1).

$\left(1^{6}\right)$ Partially order the set $P_{n}=\{(i, j): 1 \leq i<j \leq n\}$ componentwise. Then the sets $\left\{\left(i_{1}, j_{1}\right), \ldots,\left(i_{k}, j_{k}\right)\right\}$ are just the antichains of 
$P_{n}$ and hence are equinumerous with the order ideals of $P_{n}$ (see the end of Section 3.1). But $P_{n}$ is isomorphic to the poset $\operatorname{Int}(\boldsymbol{n}-\mathbf{1})$ of Exercise 6.19(bbb), so the proof follows from this exercise.

This result is implicit in the paper A. Reifegerste, European $J$. Combin. 24 (2003), 759-776. She observes that if $\left(i_{1} \cdots i_{k}, j_{1} \cdots j_{k}\right)$ is a pair being counted, then there is a unique 321-avoiding permutation $w \in \mathfrak{S}_{n}$ whose excedance set $E_{w}=\{i: w(i)>i\}$ is $\left\{i_{1}, \ldots i_{k}\right\}$ and such that $w\left(i_{k}\right)=j_{k}$ for all $k$. Conversely, every 321-avoiding $w \in \mathfrak{S}_{n}$ gives rise to a pair being counted. Thus the proof follows from Exercise 6.19(ee). Note that if we subtract 1 from each $j_{k}$, then we obtain a bijection with pairs of sequences $1 \leq i_{1}<i_{2}<\cdots<i_{k} \leq n-1$ and $1 \leq h_{1}<h_{2}<\cdots<h_{k} \leq n-1$ such that $i_{r} \leq j_{r}$ for all $r$. This variant was suggested by E. Deutsch, private communication dated 4 November 2007.

D. Callan points out (private communication, 16 January 2011) that this item is also implicit in R. Simion, J. Combinatorial Theory Ser. A 66 (1994), 270-301 (REMARK 0.2 on page 275). Namely, increment the $i$ 's by 1 and prepend a 1 to get her $f$ vector, and decrement the $j$ 's by 1 and append an $n$ to get her $\ell^{\prime}$-vector.

$\left(\mathrm{m}^{6}\right)$ If we arrange the elements of $S$ in increasing order, then we obtain the sequences of item $\mathrm{v}^{5}$.

$\left(\mathrm{n}^{6}\right)$ Let $L$ be a lattice path as in $(\mathrm{h})$. Let $(0,0)=v_{0}, v_{1}, \ldots, v_{k}=(n, n)$ be the successive points at which $L$ intersects the diagonal $y=x$. Let $L^{\prime}$ be the path obtained by reflecting about $y=x$ the portions of $L$ between each $v_{2 i-1}$ and $v_{2 i}$. The horizontal steps of $L^{\prime}$ then correspond to the moves of the first player, while the vertical steps correspond to the moves of the second player.

This result and solution are due to Lou Shapiro, private communication dated 13 May 2005. Shapiro stated the result in terms of the game of Parcheesi, but since many readers may be unfamiliar with this game we have given a more mundane formulation.

$\left(o^{6}\right)$ Since $n+1$ and $n$ are relatively prime, each equivalence class has exactly $2 n+1$ elements. Hence the number of classes is $\frac{1}{2 n+1}\left(\begin{array}{c}2 n+1 \\ n\end{array}\right)=C_{n}$. This fact is the basis for the direct combinatorial proof that there are $C_{n}$ ballot sequences (as defined in Corollary 6.2.3(ii)) of length $2 n$; see Example 5.3.12. 
$\left(\mathrm{p}^{6}\right)$ Let $C_{\lambda}=\cdots c_{-2} c_{-1} c_{0} c_{1} c_{2} \cdots$ be the code of the partition $\lambda$, as defined in Exercise 7.59, where $c_{0}=1$ and $c_{i}=0$ for $i<0$. Let $S_{\lambda}=\left\{i: c_{i}=1\right\}$. For instance, if $\lambda=(3,1,1)$ then $S_{\lambda}=\mathbb{N}-\{1,2,5\}$. It is easy to see (using Exercise $7.59(\mathrm{~b})$ ) that $\lambda$ is an $n$-core and an $(n+1)$-core if and only if $S_{\lambda}$ is a set counted by $\left(\mathrm{f}^{8}\right)$, and the proof follows. This cute result is due to J. Anderson, Discrete Math. 248 (2002), 237-243. Anderson obtains the more general result that if $m$ and $n$ are relatively prime, then the number of partitions $\lambda$ that are both $m$-cores and $n$-cores is $\frac{1}{m+n}\left(\begin{array}{c}m+m \\ m\end{array}\right)$. J. Olsson and D. Stanton (in preparation) show that in addition the largest $|\lambda|$ for which $\lambda$ is an $m$-core and $n$-core is given by $\left(m^{2}-1\right)\left(n^{2}-1\right) / 24$.

$\left(q^{6}\right)$ See D. Callan, arXiv:0802.2275, §2.4.

$\left(\mathrm{r}^{6}\right)$ We claim that each equivalence class contains a unique element $\left(a_{1}, \ldots, a_{n}\right)$ satisfying $a_{1}+a_{2}+\cdots+a_{i} \geq i$ for $1 \leq i \leq n$. The proof then follows from $\left(\mathrm{s}^{5}\right)$. To prove the claim, if $\alpha=\left(a_{1}, \ldots, a_{n}\right) \in$ $S_{n}$, then define $\alpha^{\prime}=\left(a_{1}-1, \ldots, a_{n}-1,-1\right)$. Note that the entries of $\alpha^{\prime}$ are $\geq-1$ and sum to -1 . If $E=\left\{\alpha_{1}, \ldots, \alpha_{k}\right\}$ is an equivalence class, then it is easy to see that the set $\left\{\alpha_{1}^{\prime}, \ldots, \alpha_{k}^{\prime}\right\}$ consists of all conjugates (or cyclic shifts) that end in -1 of a single word $\alpha_{1}^{\prime}$, say. It follows from Lemma 5.3.7 that there is a unique conjugate (or cyclic shift) $\beta$ of $\alpha_{1}^{\prime}$ such that all partial sums of $\beta$, except for the sum of all the terms, are nonnegative. Since the last component of $\beta$ is -1 , it follows that $\beta=\alpha_{j}^{\prime}$ for a unique $j$. Let $\alpha_{j}=\left(a_{1}, \ldots, a_{n}\right)$. Then $\alpha_{j}$ will be the unique element of $E$ satisfying $a_{1}+\cdots+a_{i} \geq i$, as desired.

A straightforward counting proof of this result appears in S. K. Pun, Higher order derivatives of the Perron root, Ph.D. thesis, Polytechnic Institute of New York University, 1994. For a generalization, see E. Deutsch and I. Gessel, Problem 10525, solution by D. Beckwith, Amer. Math. Monthl 105 (1998), 774-775.

$\left(\mathrm{s}^{6}\right)$ Let $\alpha=\left(\alpha_{1}, \ldots, \alpha_{k}\right), \beta=\left(\beta_{1}, \ldots, \beta_{k}\right)$. Define a Dyck path by going up $\alpha_{1}$ steps, then down $\beta_{1}$ steps, then up $\alpha_{2}$ steps, then down $\beta_{2}$ steps, etc. This gives a bijection with (i), due to A. Reifegerste, The excedances and descents of bi-increasing permutations, preprint (Cor. 3/8); math.CO/0212247.

$\left(\mathrm{t}^{6}\right)$ The point is that the permutations 321, 312, and 231 are them- 
selves indecomposable. It follows that if $w$ is any one of 321,312 , 231 , then the number $f(n)$ of indecomposable $w$-avoiding permutations in $\mathfrak{S}_{n}$ satisfies

$$
s_{n}(w)=\sum_{k=1}^{n} f(k) s_{n-k}(w),
$$

where $s_{m}(w)$ denotes the total number of permutations in $\mathfrak{S}_{n}$ avoiding $w$. Since $s_{n}(w)=C_{n}$, we get just as in the solution to Exercise 1.32(a) (or 1.128(a) in the second edition) that

$$
\begin{aligned}
\sum_{n \geq 1} f(n) x^{n} & =1-\frac{1}{C(x)} \\
& =x C(x)
\end{aligned}
$$

and the proof follows. For some bijective proofs see Section 4 of A. Claesson and S. Kitaev, Sém. Lotharingien de Combinatoire 60 (2008), Article B60d (electronic). [Can anyone provide the original reference?]

$\left(\mathrm{u}^{6}\right)$ For $w=213$, simply prepend a 1 to a 213-avoiding permutation of $2,3, \ldots, n+1$. For $w=132$, simply append a 4 to a 132 -avoiding permutation of $1,2, \ldots, n$. [Can anyone provide the original reference?]

$\left(\mathrm{v}^{6}\right)$ See J. Françon and G. Viennot, Discrete Math. 28 (1979), 21-35.

$\left(\mathrm{w}^{6}\right)$ Three proofs of this result are discussed by D. Gewurz and F. Merola, Europ. J. Combinatorics 27 (2006), 990-994. In particular, each of the sequences $a_{1}, \ldots, a_{n}$ and $b_{1}, \ldots, b_{n}$ uniquely determines the other. The sequences $a_{1}, \ldots, a_{n}$ are those of Exercise $6.19(\mathrm{~s})$, while the sequences $b_{1}, \ldots, b_{n}$ are the 231-avoiding permutations of $2,3, \ldots, n+1$ (equivalent to Exercise 6.19(ff)).

$\left(\mathrm{x}^{6}\right)$ This result is due to N. Reading, Clusters, Coxeter-sortable elements and noncrossing partitions, Trans. Amer. Math. Soc., to appear; math.C0/0507186. In Example 6.3 he describes the following bijection with the noncrossing partitions of (pp). The sequences $a_{1} a_{2} \cdots a_{p}$ being counted define distinct permutations $w=u_{a_{1}} u_{a_{2}} \cdots u_{a_{p}} \in \mathfrak{S}_{n}$. Define an equivalence relation $\sim$ on $[n]$ to be the transitive and reflexive closure of $w(i) \sim w(i+1)$ if 
$w(i)>w(i+1)$. The equivalence classes then form a noncrossing partition of $[n]$. For instance, if $w=143652$, then the noncrossing partition is 1-34-256.

In the special case $u_{i}=s_{i}$, there is a nice direct bijection between the sequences $a_{1} a_{2} \cdots a_{p}$ and lattice paths $L$ that never rise above $y=x$ (item (h)). Label the lattice squares by their height above the $x$-axis (beginning with height 1 ). Read the labels of the squares below the line $y=x$ and above the lattice path $L$ by first reading from bottom-to-top the leftmost diagonal, then the next leftmost diagonal, etc. This procedure sets up a bijection between (h) and the sequences $a_{1} a_{2} \cdots a_{p}$. The figure below shows a lattice path $L$ with the labeling of the lattice squares. It follows that the sequence corresponding to $L$ is 123456123413 .

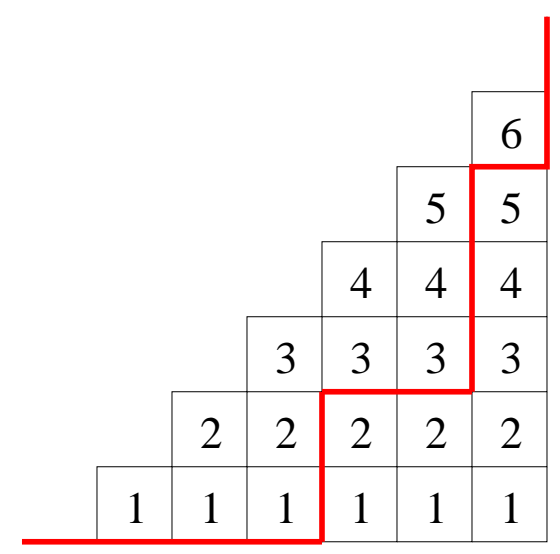

$\left(\mathrm{y}^{6}\right)$ The monomial $x_{1}^{a_{1}} \cdots x_{n}^{a_{n}}$ appears in the expansion if and only if the sequence $\left(a_{1}, \ldots, a_{n}\right)$ is enumerated by $\left(\mathrm{s}^{5}\right)$. This observation is due to E. Deutsch, private communication dated 27 September 2006.

$\left(\mathrm{z}^{6}\right)$ Replace the first occurrence of $i$ with 1 and the second occurrence with -1 to get the ballot sequences of $(r)$. This item is due to David Callan, private communication dated 1 September 2007.

$\left(\mathrm{a}^{7}\right)$ Replace the first occurrence of $i$ with 1 and the second occurrence with -1 , and then remove the first 1 and last -1 to get the ballot sequences of $(r)$. This item is due to Camillia Smith, private communication, 2008. 


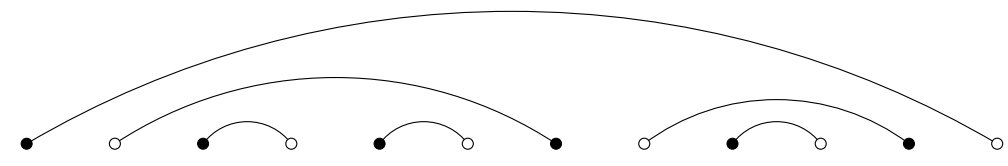

Figure 12: A noncrossing matching

$\left(\mathrm{b}^{7}\right)$ C. O. Oakley and R. J. Wisner showed (Amer. Math. Monthly 64 (1957), 143-154) that hexaflexagons can be represented by pats and deduced that they are counted by Catalan numbers. A simple bijection with plane binary trees (item (d)) was given by D. Callan, arXiv:1005.5736 (§4).

$\left(c^{7}\right)$ Deleting the first two entries (necessarily a 1 and a 2 ) and taking the positions of the first appearance of $3, \ldots, n+1$ in the resulting permutation is a bijection to (t). If we drop the condition that the first appearances of $1,2, \ldots, n+1$ occur in order, then the resulting sequences are enumerated by R. Graham and N. Zang, J. Combinatorial Theory, Ser. A 115 (2008), 293-303. This item is due to David Callan, private communication dated 1 September 2007. It is also easy to see that the sequences being enumerated coincide with those of $\left(a^{7}\right)$.

$\left(\mathrm{d}^{7}\right)$ Let $M$ be a noncrossing matching on $2 n$ vertices (item (o)). Color the vertices black and white alternating from left-to-right, beginning with a black vertex. Thus every arc connects a white vertex to a black one. If an arc connects the $i$ th black vertex to the $j$ th white vertex, then set $w(i)=j$. This sets up a bijection with the permutations being counted. For instance, if $M$ is given by Figure 12 then $w=623154$. This result was suggested by Eva Y.-P. Deng (private communication, June 2010).

$\left(\mathrm{e}^{7}\right)$ The bijection between genus 0 permutations and noncrossing partitions in the solution to Exercise 6.38(p) shows that we are counting noncrossing partitions of $[n+1]$ for which 1 is a singleton. These are clearly in bijection with noncrossing partitions of $[2, n+$ $1]$.

This item and the next three are due to E. Deutsch, (private communication, 31 May 2010).

$\left(f^{7}\right)$ Now we are counting noncrossing partitions for which 1 and 2 are in the same block. 
$\left(\mathrm{g}^{7}\right)$ Now 1 and 3 are in the same block, and 2 is a singleton.

$\left(\mathrm{h}^{7}\right)$ Now $\{1, n+2\}$ is a doubleton block.

(i $\left.{ }^{7}\right)$ See N. A. Loehr, Europ. J. Combinatorics 26 (2005), 83-93. This contrived-looking interpretation of $C_{n}$ is actually closely related to Exercise 6.25(i) and the $(q, t)$-Catalan numbers of Garsia and Haiman.

$\left(\mathrm{j}^{7}\right)$ See N. A. Loehr, ibid.

$\left(\mathrm{k}^{7}\right)$ The restriction of such $w$ to the last $n$ terms gives a bijection with 123 -avoiding permutations in $\mathfrak{S}_{n}$ (equivalent to (ee)). Namely, symmetric permutation with last $n$ entries $1, \ldots, n$ in some 123avoiding order are clearly 123-avoiding. Conversely, if there were a number $i>n+1$ among the last $n$ entries, then we would have the increasing subsequence $2 n+2-i, n+1, i$. This result (with a different proof, in a more general context) appears in E. S. Egge, Annals of Comb. 11 (2007), 405-434 (pages 407, 412).

$\left(1^{7}\right)$ Let $S_{v}(n)$ denote the set of all permutations $w \in \mathfrak{S}_{n}$ avoiding the permutation $v \in \mathfrak{S}_{k}$, and let $\operatorname{fp}(w)$ denote the number of fixed points of $w$. S. Elizalde, Proc. FPSAC 2003, arXiv :math.C0/0212221 $(q=1$ case of equation $(2))$, has shown that

$$
\sum_{n \geq 0} \sum_{w \in S_{321}(n)} x^{\mathrm{fp}(w)} t^{n}=\frac{2}{1+2 t(1-x)+\sqrt{1-4 t}} .
$$

Applying $\frac{d}{d x}$ and setting $x=1$ gives $C(t)-1$, and the proof follows. This paper also contains a bijection from 321-avoiding permutations $w$ in $\mathfrak{S}_{n}$ to Dyck paths $P$ of length $2 n$. This bijection takes fixed points of $w$ into peaks of height one of $P$. Now use $\left(\mathrm{k}^{4}\right)$ to get a bijective proof of the present item. This item is due to E. Deutsch, private communication dated 6 September 2007.

Note that the average number of fixed points of a 321-avoiding permutation in $\mathfrak{S}_{n}$ is one, the same as the average number of fixed points of all permutations in $\mathfrak{S}_{n}$. What other "interesting" classes of permutations have this property?

$\left(\mathrm{m}^{7}\right)$ Replace an excedance of $w$ with a 1 and a nonexcedance with a -1 , except for the nonexcedance $2 n+1$ at the end of $w$. This sets up a bijection with (r). There is also a close connection with 
$\left(\mathrm{o}^{5}\right)$. If $P$ is a parallelogram polyomino of the type counted by $\left(\mathrm{o}^{5}\right)$, then place $P$ in a $(2 n+1) \times(2 n+1)$ square $M$. Put a 1 in each square immediately to the right of the bottom step in each maximal vertical line on the boundary, except for the rightmost such vertical line. Put a 0 in the remaining squares of $M$. This sets up a bijection between $\left(\mathrm{O}^{5}\right)$ and the permutation matrices corresponding to the permutations counted by the present exercise. An example is given by the figure below, where the corresponding permutation is 4512736. This result is due to E. Deutsch, S. Elizalde, and A. Reifegerste (private communication, April, 2003).

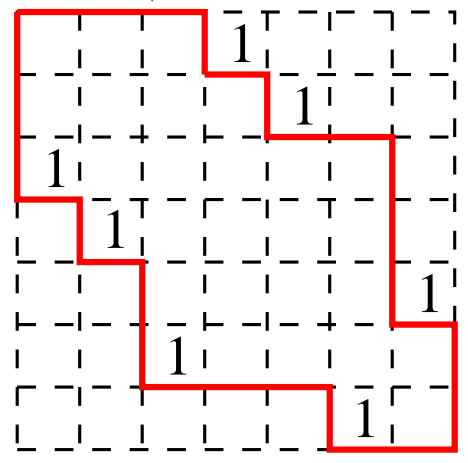

$\left(\mathrm{n}^{7}\right)$ Let $a_{1} a_{2} \cdots a_{2 n}$ be a permutation being counted, and associate with it the array

$$
\begin{array}{ccccc}
a_{2} & a_{4} & a_{6} & \cdots & a_{2 n} \\
a_{1} & a_{3} & a_{5} & \cdots & a_{2 n-1}
\end{array} .
$$

This sets up a bijection with (ww), standard Young tableaux of shape $(n, n)$. This result it due to E. Deutsch and A. Reifegerste, private communication dated 4 June 2003. Deutsch and Reifegerste also point out that the permutations being counted have an alternative description as those 321-avoiding permutations in $\mathfrak{S}_{2 n}$ with the maximum number of descents (or equivalently, excedances), namely $n$.

$\left(o^{7}\right)$ This result can be proved using the machinery of R. Stanley, J. Combinatorial Theory, Ser. A 114 (2007), 436-460. Presumably there is a simpler combinatorial proof. Note that it suffices to assume that $n$ is even, since $a_{1}, a_{2}, \ldots, a_{2 m}$ is reverse- 
alternating and 321-avoiding if and only if the same is true for $a_{1}, a_{2}, \ldots a_{2 m-1}, 2 m+1, a_{2 m}$. (By similar reasoning, item $\left(\mathrm{n}^{7}\right)$ is reduced to the case where $n$ is odd.)

Note. The results of $\left(\mathrm{n}^{7}\right)$ and $\left(\mathrm{o}^{7}\right)$ can easily be carried over to alternating 123 -avoiding and reverse alternating 123 -avoiding permutations by considering the reverse $a_{n} \cdots a_{1}$ of the permutation $a_{1} \cdots a_{n}$.

$\left(\mathrm{p}^{7}\right)$ The second entry has to be 1 . Delete it to obtain a 321-avoiding permutation of $\{2,3, \ldots, n+1\}$, enumerated by (ee). This item is due to E. Deutsch, private communication dated 18 June 2009.

$\left(\mathrm{q}^{7}, \mathrm{r}^{7}\right)$ Let $f(m)$ denote the number of alternating 132-avoiding permutations in $\mathfrak{S}_{m}$, and let $w=w_{1} w_{2} \cdots w_{m} \in \mathfrak{S}_{m}$ be such a permutation. Then $w_{2 i+1}=m$ for some $i$. Moreover, $w_{1} w_{2} \cdots w_{2 i}$ is an alternating 132-avoiding permutation of $m-2 i, m-2 i+1, \ldots, m-1$, while $w_{2 i+2}, w_{2 i+3}, \ldots, w_{m}$ is a reverse alternating permutation of $1,2, \ldots, m-2 i-1$. By induction we obtain the recurrence

$$
f(2 n)=C_{0} C_{n-1}+C_{1} C_{n-2}+\cdots+C_{n-1} C_{0},
$$

from which it follows easily that $f(2 n)=C_{n}$. The argument for $f(2 n+1)$, as well as for reverse alternating 132-avoiding permutations, is analogous. These results are due to T. Mansour, Ann. Comb. 7 (2003), 201-227, math. C0/0210058 (Theorem 2.2). Mansour obtains similar results for 132-avoiding permutations that are alternating or reverse alternating except at the first step, and he gives numerous generalizations and extensions. For further work in this area, see J. B. Lewis, Electronic J. Combinatorics 16(1) (2009), \#N7.

$\left(\mathrm{s}^{7}\right)$ By Corollary 7.13.6 (applied to permutation matrices), Theorem 7.23.17 (in the case $i=1$ ), and Exercise 7.28(a) (in the case where $A$ is a symmetric permutation matrix of trace 0 ), the RSK algorithm sets up a bijection between 321-avoiding fixed-point-free involutions in $\mathfrak{S}_{2 n}$ and standard Young tableaux of shape $(n, n)$. Now use (ww). There are also numerous ways to give a more direct bijection.

$\left(\mathrm{t}^{7}\right)$ As in $\left(\mathrm{s}^{7}\right)$, the RSK-algorithm sets up a bijection between 321avoiding involutions in $\mathfrak{S}_{2 n-1}$ with one fixed point and standard Young tableaux of shape $(n, n-1)$; and again use (ww). 
$\left(u^{7}\right)$ In the solution to (ii) it was mentioned that a permutation is stacksortable if and only if it is 231 -avoiding. Hence a permutation in $\mathfrak{S}_{2 n}$ can be sorted into the order $2 n, 2 n-1, \ldots, 1$ on a stack if and only if it is 213-avoiding. Given a 213-avoiding fixed-point-free involution in $\mathfrak{S}_{2 n}$, sort it in reverse order on a stack. When an element is put on the stack record a 1, and when it is taken off record a -1 (as in the solution to (ii)). Then we obtain exactly the sequences $a_{1}, a_{2}, \ldots, a_{n},-a_{n}, \ldots,-a_{2},-a_{1}$, where $a_{1}, a_{2}, \ldots, a_{n}$ is as in (r), and the proof follows. Moreover, E. Deutsch (private communication, May, 2001) has constructed a bijection with the Dyck paths of (i).

$\left(\mathrm{v}^{7}\right)$ Similar to $\left(\mathrm{u}^{7}\right)$.

$\left(\mathrm{w}^{7}\right)$ Obvious bijection with (ggg).

$\left(\mathrm{x}^{7}\right)$ Given a standard Young tableau $T$ of the type being counted, construct a Dyck path of length $2 n$ as follows. For each entry $1,2, \ldots, m$ of $T$, if $i$ appears in row 1 then draw an up step, while if $i$ appears in row 2 then draw a down step. Afterwards draw an up step followed by down steps to the $x$-axis. This sets up a bijection with (i).

$\left(y^{7}\right)$ The bijection of $\left(x^{7}\right)$ yields an elevated Dyck path, i.e., a Dyck path of length $2 n+2$ which never touches the $x$-axis except at the beginning and end. Remove the first and last step to get a bijection with (i).

$\left(\mathrm{z}^{7}\right)$ Remove all entries except $3,5,7, \ldots, 2 n-1$ and shift the remaining entries in the first row one square to the left. Replace $2 i+1$ with $i$. This sets up a bijection with SYT of shape $(n, n)$, so the proof follows from Exercise 6.19(ww). This result is due to T. Chow, H. Eriksson, and C. K. Fan, Chess tableaux, Electr. J. Comb. 11(2) (2004-2005), \#A3. This paper also shows the more difficult result that the number of SYT of shape $(n, n, n)$ such that adjacent entries have opposite parity is the number $B(n-1)$ of Baxter permutations of length $n-1$ (defined in Exercise 6.55).

$\left(a^{8}\right)$ These arrays encode the labelled trees of (uuu). The integer $r$ is the depth of the tree, the sequence $a_{1}, \ldots, a_{r}$ records the number of edges at each level starting at the bottom, and the sequence 


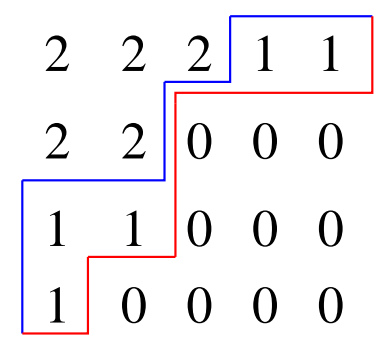

Figure 13: A plane partition and two lattice paths

$b_{1}, \ldots, b_{r-1}$ lists the non-leaf vertex labels decremented by 1 . For instance, the labelled tree

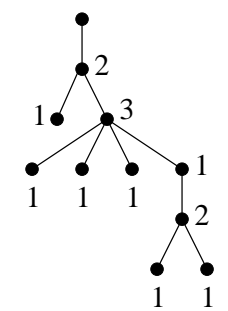

has $r=5$ and code $\left(\begin{array}{lllll}2 & 1 & 4 & 2 & 1 \\ 1 & 0 & 2 & 1 & \end{array}\right)$. This item is due to David Callan (private communication dated 19 February 2008).

$\left(\mathrm{b}^{8}\right)$ Given the plane partition $\pi$, let $L$ be the lattice path from the lower left to upper right that has only 2's above it and no 2's below. Similarly let $L^{\prime}$ be the lattice path from the lower left to upper right that has only 0's below it and no 0's above. See Figure 13 for an example. This pair of lattice paths coincides with those of Exercise 6.19(m)

$\left(c^{8}\right)$ The objects being counted are known as Catalan alternative tableaux. A bijection with plane binary trees (item (d)) was given by $\mathrm{X}$. Viennot, Catalan tableaux and the asymmetric exclusion process, Proc. FPSAC'0\%, Tianjin, China, arXiv:0905.3081. For a connection with the Hopf algebra of binary trees defined by Loday and Ronco, see J.-C. Aval and X. Viennot, Sém. Lotharingien de Combinatoire 68 (2010), article B63h.

$\left(d^{8}\right)$ See Definition 15 and Proposition 16 of K. Lee and L. Li, q,tCatalan numbers and generators for the radical ideal defining the 


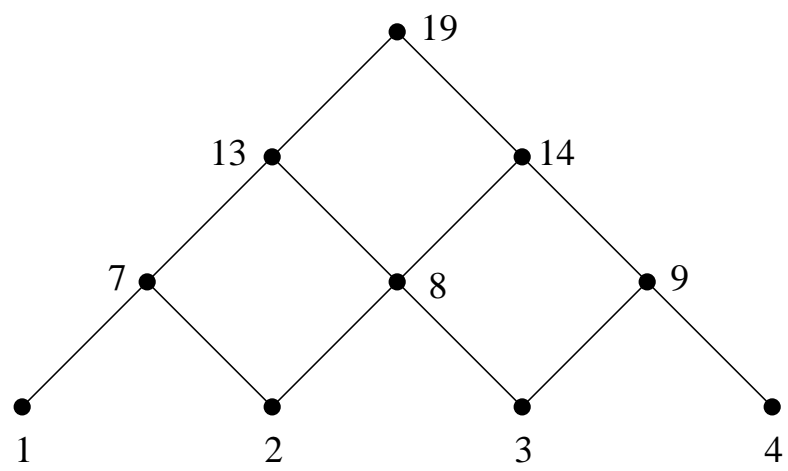

Figure 14: The poset $T_{5}$

diagonal locus of $\left(\mathbb{C}^{2}\right)^{n}$, preprint; arXiv:0909.1612. This bijection was independently found by M. Can and N. Loehr and by A. Woo.

$\left(\mathrm{e}^{8}\right)$ In the two-colored Motzkin paths of $\left(\mathrm{n}^{4}\right)$, number the steps $1,2, \ldots$, $n-1$ from left to right. Place the upsteps $(1,1)$ in $A$, the downsteps $(1,-1)$ in $B$, and the red flatsteps $(1,0)$ in $C$. This result is due to David Callan, priviate communication dated 26 February 2004 .

$\left(\mathrm{f}^{8}\right)$ Let $S_{n}$ be the submonoid of $\mathbb{N}$ (under addition) generated by $n$ and $n+1$. Partially order the set $T_{n}=\mathbb{N}-S_{n}$ by $i \leq j$ if $j-i \in S_{n}$. Figure 14 illustrates the case $n=5$. It can be checked that $T_{n} \cong \operatorname{Int}(\boldsymbol{n} \mathbf{- 1})$, as defined in Exercise 6.19(bbb). Moreover, the subsets $S$ being counted are given by $\mathbb{N}-I$, where $I$ is an order ideal of $T_{n}$. The proof follows from Exercise 6.19(bbb). This result is due to Mercedes H. Rosas, private communication dated 29 May 2002.

$\left(\mathrm{g}^{8}\right)$ Let $S_{i}-S_{i-1}=\left\{a_{i}\right\}$. Then the sequences $a_{1} a_{2} \cdots a_{n}$ coincide with the 312-avoiding permutations of (ff). Communication from $\mathrm{R}$. Proctor dated 12 June 2012.

$\left(\mathrm{h}^{8}\right)$ Analogous to $(\mathrm{jjj})$, using $\frac{1}{n}\left(\begin{array}{c}2 n \\ n+1\end{array}\right)=C_{n}$. This problem was suggested by S. Fomin.

(i $\left.{ }^{8}\right)$ Let $a_{i}$ be the multiplicity of $e_{i}-e_{i+1}$ in the sum. The entire sum is uniquely determined by the sequence $a_{1}, a_{2}, \ldots, a_{n-1}$. Moreover, the sequences $0, a_{1}, a_{2}, \ldots, a_{n-1}$ that arise in this way coincide with 
those in $(\mathrm{u})$. This exercise is an unpublished result of A. Postnikov and R. Stanley.

$\left(\mathrm{j}^{8}\right)$ Let $H$ be a polyomino of the type being counted, say with $\ell$ rows. Let $a_{i}+1$ be the width (number of columns) of the first $i$ rows of $H$, and let $\alpha_{i}=a_{i}-a_{i-1}$ for $1 \leq i \leq \ell$ (with $a_{0}=0$ ). Similarly let $b_{i}+1$ be the width of the last $i$ rows of $H$, and let $\beta_{i}=b_{\ell-i+1}-b_{\ell-i}$ for $1 \leq i \leq \ell$ (with $b_{0}=1$ ). This sets up a bijection with the pairs $(\alpha, \beta)$ of compositions counted by $\left(\mathrm{s}^{6}\right)$. This argument is due to A. Reifegerste, ibid. By a refinement of this argument she also shows that the number of polyominoes of the type being counted with $\ell$ rows is the Narayana number $N(n, \ell)=\frac{1}{n}\left(\begin{array}{c}n \\ \ell\end{array}\right)\left(\begin{array}{c}n \\ \ell-1\end{array}\right)$ of Exercise 6.36. Another bijection was provided by E. Deutsch (private communication dated 15 June 2001). Namely, given the polynomino $H$, let $a_{1}+1, \ldots, a_{\ell}+1$ be the row lengths and $b_{1}+1, \ldots, b_{\ell-1}+1$ be the lengths of the overlap between the successive rows. Let $D$ be the Dyck path of length $2 n$ with successive peaks at heights $a_{1}, \ldots, a_{\ell}$ and successive valleys at heights $b_{1}, \ldots, b_{\ell-1}$. This sets up a bijection with Dyck paths of length $2 n$, as given in (i). (Compare with the solution to (l).)

$\left(\mathrm{k}^{8}\right)$ There is a simple bijection with the binary trees $T$ of $(\mathrm{c})$. The root of $T$ corresponds to the rectangle containing the upper righthand corner of the staircase. Remove this rectangle and we get two smaller staircase tilings, making the bijection obvious. This result is the case $d=2$ of Theorem 1.1 of H. Thomas, Order 19 (2002), 327-342.

$\left(1^{8}\right)$ Concatenate the nonhorizontal edges of the matching and adjoin an up step at the beginning and down step at the end to get a Dyck path as in (i). See Figure 15 for an example. This result is due to S. J. Cyvin and I. Gutman, Kekulé Structures in Benzenoid Hydrocarbons, Lecture Notes in Chemistry 46, Springer-Verlag, Berlin, 1988. The proof given here appears in the interesting survey by T. Došlić, Croatica Chemica Acta 78(2) (2005), 251-259 (Prop. 5.2).

$\left(\mathrm{m}^{8}\right)$ Such posets are known as series-parallel interval orders. They are enumerated by F. Disanto, L. Ferrari, R. Pinzani, and S. Rinaldi, Catalan numbers and relations, preprint. To obtain a bijection 

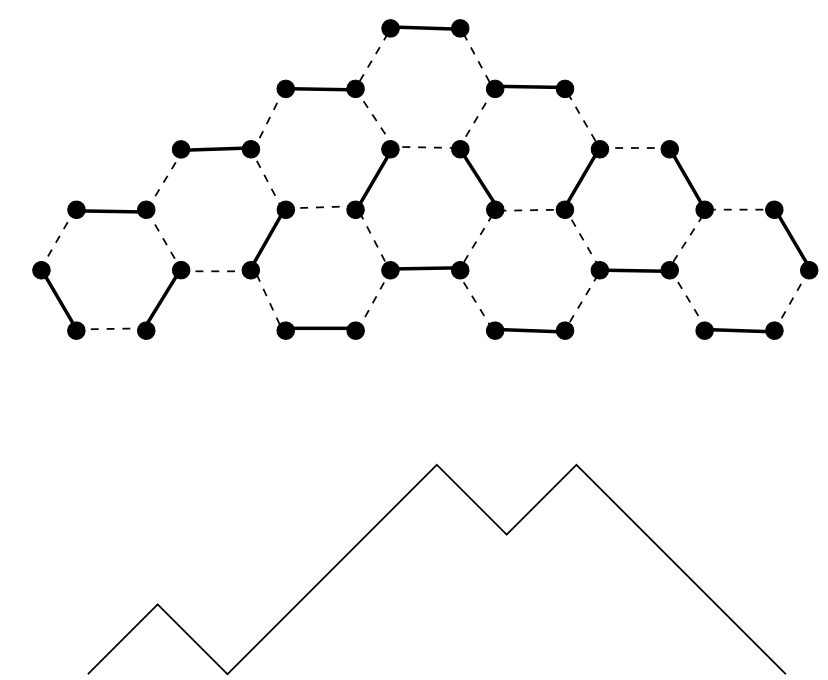

Figure 15: A matching on $T_{4}$ and the corresponding Dyck path

with (o) (noncrossing matchings), let $M$ be a noncrossing matching on $[2 n]$. Define a partial order on the arcs by letting $a<b$ if $a$ lies entirely to the left of $b$.

$\left(\mathrm{n}^{8}\right)$ Let $A$ be an antichain of the poset of intervals of the chain $\boldsymbol{n}-\mathbf{1}$. The number of such antichains is $C_{n}$ by (bbb), since for any poset there is a simple bijection between its order ideals $I$ and antichains $A$, viz., $A$ is the set of maximal elements of $I$. (See equation (2) of Section 3.1.) Construct from $A$ a poset $P$ on the points $1,2, \ldots, n+1,1^{\prime}, 2^{\prime}, \ldots,(n+1)^{\prime}$ as follows. First, $1<2<\cdots<$ $n+1$ and $1^{\prime}<2^{\prime}<\cdots<(n+1)^{\prime}$. If $[i, j] \in A$, then define $i<$ $(j+2)^{\prime}$ and $j<(i+2)^{\prime}$. This gives a bijection between (bbb) and $\left(\mathrm{n}^{8}\right)$. This result is due to J. Stembridge, private communication dated 22 November 2004.

$\left(o^{8}\right)$ Let $w \in \mathfrak{S}_{n}$ be 213-avoiding (equivalent to (ff)). Let $\varphi(w)=$ $\{(i, j): i<j, w(i)<w(j)\}$. This sets up a bijection with the set of pairs $i<_{P} j$ for one of the posets $P$ being enumerated. This item (stated differently) is due to $\mathrm{S}$. Sam, private communication dated 23 October 2009.

$\left(\mathrm{p}^{8}\right)$ A. Claesson and S. Linusson, Proc. Amer. Math. Soc. 139 (2011), 435-449, describe on page 445 a bijection with nonnesting matchings (item $\mathrm{e}^{5}$ ). They also point out that no two of the posets being 
counted are isomorphic (as unlabelled posets), and that the unlabelled posets appearing here are just the semiorders (i.e., $(\mathbf{3}+\mathbf{1})$ free and $\mathbf{2}+\mathbf{2}$ )-free posets) on $n$ vertices of item (ddd).

$\left(q^{8}\right)$ These are just the 321-avoiding permutations $w \in \mathfrak{S}_{n}$ of item (ee). This result appears (without explicitly stating that the answer is $C_{n}$ ) as Exercise 3.185(i) (second edition).

$\left(\mathrm{r}^{8}\right)$ Call a subset $T \subseteq I_{m}$ satisfying the condition a valid $m$-set. Write $D_{m}$ for the number of valid $m$-sets. Let $S$ be a valid $(n+1)$-set. Let $i$ be the least integer for which $(i, 0) \in S$. (Set $i=n$ if there is no such integer.) The set $J_{1}=\left\{(h, k) \in I_{n+1}: h>i\right\}$ is a poset isomorphic to $I_{n-i}$. Choose a valid $(n-i)$-set $S_{1}$ in $J_{1}$ after identifying it with $I_{n-i}$. Similarly $J_{2}=\left\{(h, k) \in I_{n+1}: k>n-i\right\}$ is isomorphic to $I_{i}$. Choose a valid $(n-i)$-set $S_{2}$ in $J_{2}$ after identifying it with $I_{i}$. It can be checked that there is a unique subset $T$ of $I_{n+1}-J_{1}-J_{2}$ for which $S_{1} \cup S_{2} \cup T$ is a valid $(n+1)$ set. There are $D_{n-i}$ choices for $S_{1}$ and $D_{i}$ choices for $S_{2}$, so

$$
D_{n+1}=\sum_{i=0}^{n} D_{i} D_{n-i}
$$

This recurrence, together with the initial condition $D_{0}=1$, shows that $D_{n}=C_{n}$.

This result was obtained (with a more geometric description) by J. Stevens, arXiv:0906.1430 (Theorem 3), in connection with versal deformations of cyclic quotient singularities.

$\left(s^{8}\right)$ This result is due to F. Disanto, L. Ferrari, R. Pinzani, and S. Rinaldi, Catalan numbers and relations, preprint. To obtain a bijection with (o) (noncrossing matchings), let $M$ be a noncrossing matching on $[2 n]$. Define binary relations $S$ and $R$ on the arcs of $M$ as follows. Let $a S b$ if $a$ lies underneath $b$, and define $a R b$ if $a$ lies entirely to the left of $b$. We get all nonisomorphic pairs $(S, R)$ under consideration exactly once, though this takes some work to prove.

$\left(t^{8}\right)$ Let

$(1,1, \ldots, 1,-n)=\sum_{i=1}^{n-1}\left(a_{i}\left(e_{i}-e_{i+1}\right)+b_{i}\left(e_{i}-e_{n+1}\right)\right)+a_{n}\left(e_{n}-e_{n+1}\right)$ 
as in $\left(\mathrm{i}^{8}\right)$. Set $m_{i i}=a_{i}$ and $m_{i n}=b_{i}$. This uniquely determines the matrix $M$ and sets up a bijection with $\left(i^{8}\right)$. This result is due to A. Postnikov and R. Stanley (unpublished).

$\left(\mathrm{u}^{8}\right)$ See R. Johansson and S. Linusson, Ann. Combinatorics 11 (2007), 471-480 (Corollary 3.4). This result is proved by specializing the bijection used to prove Exercise 6.39(w), thereby setting up a bijection with $\left(\mathrm{v}^{4}\right)$.

$\left(\mathrm{v}^{8}\right)$ The solution to (lll) sets up a bijection between order ideals of Int $(\boldsymbol{n}-\mathbf{1})$ and all regions into which the cone $x_{1} \geq x_{2} \geq \cdots \geq x_{n}$ is divided by the hyperplanes $x_{i}-x_{j}=1$, for $1 \leq i<j \leq n$. In this bijection, the bounded regions correspond to the order ideals containing all singleton intervals $[i, i]$. It is easy to see that such order ideals are in bijection with all order ideals of $\operatorname{Int}(\boldsymbol{n}-\mathbf{2})$. Now use (bbb). This result was suggested by S. Fomin.

$\left(\mathrm{w}^{8}\right)$ Given $w=b_{1} b_{2} \cdots b_{n} \in \mathfrak{S}_{n}$, let $c_{i}=\min \left\{b_{i}, b_{i+1}, \ldots, b_{n}\right\}$ and $c(w)=\left(c_{1}, \ldots, c_{n}\right)$. Then

$$
\operatorname{vol}\left(X_{w}\right)=\int_{x_{b_{1}}=0}^{c_{1}} \int_{x_{b_{2}}=x_{b_{1}}}^{c_{2}} \cdots \int_{x_{b_{n}}=x_{b_{n-1}}}^{c_{n}} d x_{1} d x_{2} \cdots d x_{n} .
$$

Hence $\operatorname{vol}\left(X_{v}\right)=\operatorname{vol}\left(X_{w}\right)$ if $c(v)=c(w)$, and it is not difficult to see that the converse holds. It follows that the number of distinct volumes is the number of distinct sequences $c(w), w \in \mathfrak{S}_{n}$. These are just the sequences $\left(c_{1}, \ldots, c_{n}\right) \in \mathbb{P}^{n}$ satisfying $c_{1} \leq c_{2} \leq \cdots \leq$ $c_{n}$ and $c_{i} \leq i$, and the proof follows from (s). The problem of finding the number of distinct volumes was raised by Knuth. A solution was given by B. Young, A solution to one of Knuth's permutation problems, arXiv:1004.4159.

$\left(\mathrm{x}^{8}\right)$ See L. J. Billera and G. Hetyei, J. Combinatorial Theory, Ser. A 89 (2000), 77-104; math. C0/9706220 (Corollary 4).

$\left(y^{8}\right)$ Private communication from M. Aguiar dated 22 February 2013. To get a bijection with (i) (Dyck paths), associate a Dyck path $f(w)$ with an element $y$ of $N_{1}$, written with the maximum possible number of $x$ 's, as follows. Begin with $f(\emptyset)=\emptyset$. If $y=y_{1}+y_{2}$, then set $f(y)=f\left(y_{1}\right) f\left(y_{2}\right)$ (concatenation of paths). If $y=x z$, then set $f(y)=U f(z) D$. 
6.25 (j) The degree of $G(k, n+k)$ is the number $f^{\left(n^{k}\right)}$ of standard Young tableaux of the rectangular shape $\left(n^{k}\right)$ (see e.g. R. Stanley, Lecture Notes in Math. 579, Springer, Berlin, 1977, pp. 217-251 (Thm. 4.1) or L. Manivel, Symmetric Functions, Schubert Polynomials and Degeneracy Loci, American Mathematical Society and Société Mathématique de France, 1998), and the proof follows from Exercise 6.19(ww).

(k) This result was conjectured by J.-C. Aval, F. Bergeron, N. Bergeron, and A. Garsia. The "stable case" (i.e., $n \rightarrow \infty$ ) was proved by J.-C. Aval and N. Bergeron, Catalan paths, quasi-symmetric functions and super-harmonic spaces, preprint; math.C0/0109147. The full conjecture was proved by J.-C. Aval, F. Bergeron, and N. Bergeron, Ideals of quasi-symmetric functions and super-covariant polynomials for $S_{n}$, preprint; math. C0/0202071.

(1) This is a result of Alexander Woo, math.C0/0407160. Woo conjectures that $\Omega_{w}$ is the "most singular" Schubert variety, i.e., the point $X_{w_{0}}$ (which always has the largest multiplicity for any Schubert variety $\Omega_{v}$ ) of $\Omega_{w}$ has the largest multiplicity of any point on any Schubert variety of $\operatorname{GL}(n, \mathbb{C}) / B$.

(m) A matrix $A \in \operatorname{SL}(n, \mathbb{C})$ satisfying $A^{n+1}=1$ is diagonalizable with eigenvalues $\zeta$ satisfying $\zeta^{n+1}=1$. The conjugacy class of $A$ is then determined by its multiset of eigenvalues. It follows that the number of conjugacy classes is the number of multisets of $\mathbb{Z} /(n+$ $1) \mathbb{Z}$ whose elements sum to 0 . Now use (jjj). For the significance of this result and its generalization to other Lie groups, see D. Z. Djokovíc, Proc. Amer. Math. Soc. 80 (1980), 181-184. Further discussion appears in Lecture 5 of S. Fomin and N. Reading, Root systems and generalized associahedra, available at

$$
\text { Www.math. Isa.umich. edu/ fomin/Papers. }
$$

(n) The determinant of a square submatrix $B$ of $A$ will not vanish if and only if the main diagonal entries of $B$ are nonzero. Numerous simple bijections are now available to complete the proof.

(o) This result can be derived from D. G. Mead, Pacific J. Math. 42 (1972), 165-175 (proof of Lemma 1); J. Désarménien, J. P. S. Kung, and G.-C. Rota, Advances in Math. 27 (1978), 63-92 (proof of 
Theorem 2.2); and P. Doublilet, G.-C. Rota, and J. Stein, Studies in Applied Math. 53 (1974), 185-216 (proof of Theorem 1). The relevant idea is more explicit in J. Désarménien, Discrete Math. 30 (1980), 51-68 (Theorem 2). An explicit statement and proof appears in B. Rhoades and M. Skandera, Ann. Combinatorics 9 (2005), 451-495 (Proposition 4.7). (I am grateful to Mark Skandera for providing these references.)

(p) By definition of matrix multiplication, we have

$$
\left(A^{2 n}\right)_{11}=\sum a_{1 i_{1}} a_{i_{1} i_{2}} \cdots a_{i_{2 n-1,1}} .
$$

Each nonzero term in this sum is equal to 1 . There is a bijection between such terms and the ballot sequence of (r), given by associating the term $a_{1 i_{1}} a_{i_{1} i_{2}} \cdots a_{i_{2 n-1} 1}$ with the ballot sequence

$$
i_{1}-1, i_{2}-i_{1}, i_{3}-i_{2}, \ldots, 1-i_{2 n-1} \text {. }
$$

For a vast generalization, see P. Monsky, Electronic J. Combinatorics 18(1) (2011), P5.

6.38 (n) See O. Guibert, E. Pergola, and R. Pinzani, Ann. Combinatorics 5 (2001), 153-174.

(o) Let $f_{n}$ be the number of such trees on $n$ vertices. Then

$$
f_{n+1}=\sum_{k-1}^{n} f_{k} f_{n-k}, \quad n \geq 1 .
$$

Setting $y=\sum_{n \geq 0} f_{n} x^{n}$ we obtain

$$
\frac{y-1-x}{x}=y^{2}-y
$$

It follows that

$$
y=\frac{1+x-\sqrt{1-2 x-3 x^{2}}}{2 x}
$$

Comparing with the definition of $M_{n}$ in Exercise 6.27 shows that $f_{n}=M_{n-1}, n \geq 1$. It shouldn't be difficult to give a bijective proof. 
(p) It is known (e.g., Lemma 2.1 of S. Dulucq and R. Simion, J. Alg. Comb. 8 (1998), 169-191) that a permutation $w \in \mathfrak{S}_{n}$ has genus 0 if and only if each cycle of $w$ is increasing (when the smallest element of the cycle is written first) and the sets of elements of the cycles (i.e., the orbits of $w$ ) form a noncrossing partition of $[n]$. It follows that both $w$ and $w^{-1}$ have genus 0 if and only if $w$ is an involution whose cycle elements form a noncrossing (partial) matching. The proof now follows from 6.38(a). This item is due to E. Deutsch, private communication dated 26 August 2009.

Note. The number of permutations $w \in \mathfrak{S}_{n}$ of genus 0 is $C_{n}$ (item (hh)). The solution to (hh) does not mention the elegant characterization in terms of noncrossing partitions.

6.39 (t) Rotate the paths of Exercise 6.39(j) $45^{\circ}$ clockwise, reflect about the $x$-axis, and double coordinates. Both the paths of Exercise 6.39(j) and the present item are known as (large) Schröder paths.

(u) Let $L$ be a path counted by (t) with no level steps on the $x$-axis. For every up step $s$ beginning at the $x$-axis, let $t$ be the first down step after $s$ ending at the $x$-axis. Remove the steps $s$ and $t$, lower the portion of $L$ between $s$ and $t$ by one unit, and append a step $(2,0)$ at the end of this lowered portion of $L$. We obtain a bijection between paths counted by the present exercise and those paths in (t) with at least one level step on the $x$-axis. Hence the total number paths counted by the present exercise is $\frac{1}{2} r_{n}=s_{n}$. The paths of this item are known as small Schröder paths.

(v) Let $f(n)$ be the number of guillotine rectangulations being counted. Exactly $f(n) / 2$ guillotine rectangulations contain a vertical line segment cutting $R$ into two rectangles. By considering the first point $p_{i}$ left-to-right through which such a vertical line segment passes, we obtain the recurrence [why?]

$$
\frac{1}{2} f(n)=f(n-1)+\sum_{k=2}^{n}\left(\frac{1}{2} f(k-1)\right) f(n-k) .
$$

It is straightforward to obtain from this recurrence that $f(n)=$ $r_{n}$. This result is due to E. Ackerman, G. Barequet, and R. Y. 
Pinter, J. Combinatorial Theory, Ser. A 113 (2006), 1072-1091 (Theorem 2).

(w) See R. Johansson and S. Linusson, Ann. Combinatorics 11 (2007), 471-480 (§3.4), where a bijection is given with $6.39(\mathrm{j})$.

6.C1 It is not difficult to see that the term indexed by the composition $a_{0}+a_{1}+\cdots+a_{k}$ is the number of sequences counted by item $\left(\mathrm{a}^{6}\right)$ such that $i$ appears $a_{i}$ times. This result is due to D. Zare,

http://mathoverflow. net/questions/131585.

6.C2 (a) These results appear in M. Bousquet-Mélou and G. Schaeffer, Probab. Theory Related Fields 124 (2002), 305-344; math.CO/0012230

(Theorem 7), and M. Bousquet-Mélou, Walks on the slit plane: other approaches, Adv. in Applied Math., 27 (2001), 243-288; math.CO/0104111 (Theorem 19). The proofs are obtained from the formula

$$
\begin{aligned}
& \sum_{\substack{n \geq 0 \\
\sum_{i, j}}} a_{i, j}(n) x^{i} y^{j} t^{n}= \\
& \frac{(1-2 t(1+\bar{x})+\sqrt{1-4 t})^{1 / 2}(1+2 t(1-\bar{x})+\sqrt{1+4 t})^{1 / 2}}{2(1-t(x+\bar{x}+y+\bar{y}))}
\end{aligned}
$$

where $\bar{x}=1 / x$ and $\bar{y}=1 / y$. Equation (1) is also given a bijective proof in the second paper (Proposition 2).

(b) Equation (2) was conjectured by Bousquet-Mélou and Schaeffer, ibid., §3.1. This conjecture, as well as equation (3), was proved by G. Xin, Discrete Math. 282 (2004), 281-287; math. C0/0304178. The proof is obtained from (11) as in (a).

(c) In fact, these are just the 2413 and 3142-avoiding permutations (also called separable permutations) included in Exercise 6.39(l). See E. Ghys, Amer. Math. Monthly 120 (2013), 232-242.

(d) The situation is analogous to (a). The results appear in the two papers cited in (a) and are based on the formula

$$
\sum_{n \geq 0} \sum_{i, j} b_{i, j}(n) x^{i} y^{j} t^{n}=\frac{\left(1-8 t^{2}\left(1+\bar{x}^{2}\right)+\sqrt{1-16 t^{2}}\right)^{1 / 2}}{\sqrt{2}(1-t(x+\bar{x})(y+\bar{y}))} .
$$


The case $i=1$ of (4) is given a bijective proof in Bousquet-Mélou and Schaeffer, ibid., Proposition 7.

(e) Let $X_{n}$ be the set of all closed paths of length $2 n$ from $(0,0)$ to $(0,0)$ that intersect the half-line $L$ defined by $y=x, x \geq 1$. Given $P \in X_{n}$, let $k$ be the smallest integer such that $P$ intersects $L$ after $k$ steps, and let $Q$ be the path consisting of the first $k$ steps of $P$. Let $P^{\prime}$ be the path obtained from $P$ by reflecting $Q$ about the line $y=x$. Then $P^{\prime} \in X_{n}, w\left(P^{\prime}\right)=w(P) \pm 1$, and the map $P \mapsto P^{\prime}$ is an involution. Any path $P$ of length $2 n$ from $(0,0)$ to $(0,0)$ not contained in $X_{n}$ satisfies $w(P)=0$. It follows that $f(n)$ is the number of closed paths of length $2 n$ from $(0,0)$ to $(0,0)$ not intersecting $L$.

Now consider the linear change of coordinates $(x, y) \mapsto \frac{1}{2}(-x-$ $y, x-y)$. This transforms a closed path of length $2 n$ from $(0,0)$ to $(0,0)$ not intersecting $L$ to a closed path from $(0,0)$ to $(0,0)$ with $2 n$ diagonal steps $( \pm 1, \pm 1)$, not intersecting the negative real axis. Now use equation (5).

This result was stated by R. Stanley, Problem 10905, Problems and Solutions, Amer. Math. Monthly 108 (2001), 871. The published solution by R. Chapman, 110 (2003), 640-642, includes a self-contained proof of equation (5).

6.C3 (a) The enumeration of rooted planar maps subject to various conditions is a vast subject initiated by $\mathrm{W}$. T. Tutte. The result of this problem appears in Canad. J. Math. 15 (1963), 249-271. A good introduction to the subject, with many additional references, can be found in $[3.16, \S 2.9]$. There has been a revival of interest in the enumeration of maps, motivated in part by connections with physics. At present there is no comprehensive survey of this work, but two references that should help combinatorialists get into the subject are D. M. Jackson, Trans. Amer. Math. Soc. 344 (1994), 755-772, and D. M. Jackson, in DIMACS Series in Discrete Mathematics and Computer Science 24 (1996), 217-234.

(b) Let $G$ be a rooted planar map with $n$ edges and $p$ vertices. We can define a dual $n$-edge and $p^{\prime}$-vertex map $G^{\prime}$, such that by Euler's formula $p^{\prime}=n+2-p$. From this it follows easily that the answer is given by $M(n)(n+2) / 2$, where $M(n)$ is the answer to (a). 
For a more general result of this nature, see V. A. Liskovets, J. Combinatorial Theory, Ser. B 75 (1999), 116-133 (Prop. 2.6). It is also mentioned on page 150 of L. M. Koganov, V. A. Liskovets and T. R. S. Walsh, Ars Combinatoria 54 (2000), 149-160.

(c) This result was first proved nonbijectively by R. C. Mullin, Canad. J. Math. 19 (1967), 174-183. The first bijective proof was given by R. Cori, S. Dulucq, and G. Viennot, J. Combinatorial Theory, Ser. A 43 (1986), 1-22. A nicer bijective proof was given by O. Bernardi, Electr. J. Comb. 124 (2007), \#R9.

6.C4 (a) See T. Nakamigawa, Theoretical Computer Science 235 (2000), 271-282 (Corollary 6) and A. Dress, J. Koolen, and V. Moulton, European J. Combin. 23 (2002), 549-557. Another proof was given by J. Jonsson in the reference cited below.

(b) See J. Jonsson, J. Combinatorial Theory, Ser. A 112 (2005), 117142

http://www.math.kth.se/ jakobj/combin.html\#deltank.

The proof interprets the result in terms of lattice path enumeration and applies the Gessel-Viennot theory of nonintersecting lattice paths $(\S 2.7)$.

(c) See L. Serrano and C. Stump, Electr. J. Comb. textbf19 (2012), P16.

6. C5 This result was first proved by I. Dumitriu using random matrix arguments. An elegant bijective proof was then given by E. Rassart, as follows. We want a bijection $\varphi$ from (1) quadruples $\left(D, D^{\prime}, e, e^{\prime}\right)$, where $D$ and $D^{\prime}$ are Dyck paths with $2 n$ steps and $e$ and $e^{\prime}$ are up edges of $D$ and $D^{\prime}$ ending at the same height $i$, and (2) Dyck paths $E$ with $4 n$ steps from $(0,0)$ to $(4 n, 0)$ which do not touch the point $(2 n, 0)$. We first transform $(D, e)$ into a partial Dyck path $L$ ending at height $i$. Let $f$ be the down edge paired with $e$ (i.e., the first down edge after $e$ beginning at height $i$ ), and flip the direction of each edge at or after $f$. Let $e_{2}$ be the first up edge to the left of $e$ ending at height $i-1$, and let $f_{2}$ be the down edge paired with $e_{2}$ in the original path $D$. Flip all edges of the current path at or after $f_{2}$. Continue this procedure, letting $e_{j}$ be the first edge to the left of $e_{j-1}$ such that $e_{j}$ has height 

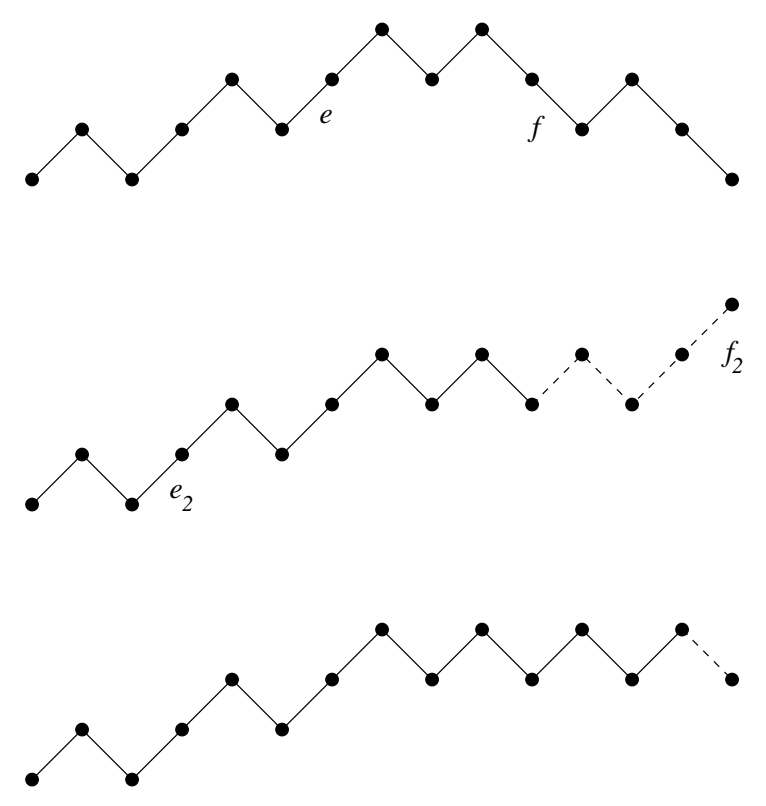

Figure 16: The bijection $(D, e) \mapsto L$ in the solution to Exercise 6.C5

one less than $e_{j-1}$, etc., until no edges remain. We obtain the desired partial Dyck path $L$ ending at height $i$. Do the same for $\left(D^{\prime}, e^{\prime}\right)$, obtaining another partial Dyck path $L^{\prime}$ ending at height $i$. Reverse the direction of $L^{\prime}$ and glue it to the end of $L$. This gives the Dyck path $E$. Figure 16 shows an example of the correspondence $(D, e) \mapsto L$. We leave the construction of $\varphi^{-1}$ to the reader. See I. Dumitriu and E. Rassart, Electr. J. Comb. 10(1) (2003), R-43.

6.C6 See S. Choi and H. Park, A new graph invariant arises in toric topology, preprint; arXiv:1210.3776 (Theorem 2.5) for the case $k=2$.

6.C7 This result (in a much more general context) is due to B. Drake, Ph.D. thesis, Brandeis University, 2008 (Example 1.9.3). A bijective proof was found by Sharon J. X. Hou.

6.C8 Private communication from Vasu Tewari, 18 February 2011.

6.C9 (a) The most straightforward method is to observe that all three power series $F\left(t_{0}, t_{1}, \ldots ; x\right)$ satisfy

$$
F\left(t_{0}, t_{1}, \ldots ; x\right)=\frac{1}{1-t_{0} x F\left(t_{1}, t_{2}, \ldots ; x\right)}
$$


with the initial condition

$$
F\left(t_{0}, t_{1}, \ldots ; 0\right)=1 \text {. }
$$

A general combinatorial theory of continued fractions is due to $\mathrm{P}$. Flajolet, Discrete Math. 32 (1980), 125-161. The equivalence of (i) and (iii) is equivalent to a special case of Corollary 2 of this paper.

(b) Put $t_{0}=t_{1}=\cdots=t_{n}=1$ and $t_{i}=0$ for $i>n$. By the case $j=2$ of Exercise 1.3, the generating function of part (ii) above becomes the left-hand side of equation (7). On the other hand, by Exercise 6.19(e) the coefficient of $x^{i}$ for $i \leq n+1$ in the generating function of part (iii) becomes the coefficient of $x^{i}$ in the right-hand side of (7) (since a tree with $i \leq n+1$ vertices has height at most $n)$. Now let $n \rightarrow \infty$.

It is not difficult to give a direct proof of (7). The generating function $F_{n}(x)=\sum_{i \geq 0}(-1)^{i}\left(\begin{array}{c}n-i \\ i\end{array}\right) x^{i}$ satisfies

$$
F_{n+2}(x)=F_{n+1}(x)-x F_{n}(x) .
$$

From Theorem 4.1.1 (or directly from Exercise 6.C19(b)) it follows that

$$
F_{n}(x)=\frac{C(x)^{-n-1}-(x C(x))^{n+1}}{\sqrt{1-4 x}} .
$$

Hence the fraction in the left-hand side of (7) is given by

$$
\frac{\sum_{i \geq 0}(-1)^{i}\left(\begin{array}{c}
n-i \\
i
\end{array}\right) x^{i}}{\sum_{i \geq 0}(-1)^{i}\left(\begin{array}{c}
n+1-i \\
i
\end{array}\right) x^{i}}=C(x) \frac{1-x^{n+1} C(x)^{2(n+1)}}{1-x^{n+2} C(x)^{2(n+2)}},
$$

and (7) follows.

Equation (7) (with the numerator and denominator on the lefthand side defined by (12)) was given by V. E. Hoggatt, Jr., Problem H-297, Fibonacci Quart. 17 (1979), 94; solution by P. S. Bruckman, 18 (1980), 378.

(c) This result is due to A. Postnikov and B. Sagan, J. Combinatorial Theory, Ser. A 114 (2007), 970-977. It was originally conjectured by Postnikov for the case $t_{i}=(2 i+1)^{2}$. A $q$-analogue is due to M. Konvalinka, J. Combinatorial Theory, Ser. A 114 (2007), 10891100. 
6.C10 These are unpublished results of A. Postnikov and R. Stanley. For proofs, variations, and strengthenings, see K. Mészáros, arXiv:0904.2194 and arXiv:0904.3339.

6.C11 This result was conjectured by F. Brenti in 1995 and first proved by D. Zeilberger,

\section{http://www . math.rutgers . edu/ zeilberg/mamarim/ mamarimhtml/catalan.html.}

Zeilberger's proof consists essentially of the statement

$$
\begin{aligned}
B_{2 n}(q)= & (-1)^{n} \frac{1}{2 n+1}\left(\begin{array}{c}
2 n+1 \\
n
\end{array}\right) q^{n} \\
& +\sum_{i=0}^{n-1}(-1)^{i} \frac{2 n-2 i+1}{2 n+1}\left(\begin{array}{c}
2 n+1 \\
i
\end{array}\right)\left(q^{i}+q^{2 n-i}\right) \\
B_{2 n+1}(q)= & \sum_{i=0}^{n}(-1)^{i+1} \frac{n-i+1}{n+1}\left(\begin{array}{c}
2 n+2 \\
i
\end{array}\right) q^{i} .
\end{aligned}
$$

Later (as mentioned by Zeilberger, ibid.) Brenti found a combinatorial interpretation of the polynomials $B_{m}(q)$ which implies his conjecture.

6.C12 (a,c) Let $M^{\prime}$ denote the part of $M$ below the main diagonal. It is easy to see that $M^{\prime}$ uniquely determines $M$. It was shown by $\mathrm{C}$. S. Chan, D. P. Robbins, and D. S. Yuen, Experiment. Math. 9 (2000), 91-99, that the polytope $\mathrm{CR}_{n+1}$ (called the Chan-Robbins polytope or Chan-Robbins-Yuen polytope) can be subdivided into simplices, each of relative volume $1 /\left(\begin{array}{c}n+1 \\ 2\end{array}\right)$ !, which are naturally indexed by the matrices $M^{\prime}$. It follows that $\left(\begin{array}{c}n+1 \\ 2\end{array}\right) ! \nu\left(\mathrm{CR}_{n+1}\right)=g(n)$. Chan and Robbins had earlier conjectured that $\left(\begin{array}{c}n+1 \\ 2\end{array}\right) ! \nu\left(\mathrm{CR}_{n+1}\right)=$ $C_{1} C_{2} \cdots C_{n}$. (Actually, Chan and Robbins use a different normalization of relative volume so that $\nu\left(\mathrm{CR}_{n+1}\right)=C_{1} C_{2} \cdots C_{n}$.) This conjecture was proved by D. Zeilberger, Electron. Trans. Numer. Anal. 9 (1999), 147-148, and later W. Baldoni-Silva and M. Vergne, Residue formulae for volumes and Ehrhart polynomials of convex polytopes, preprint; math.CO/0103097 (Thm. 33). 
(b) Given the matrix $M=\left(m_{i j}\right)$, there exist unique nonnegative integers $a_{1}, \ldots, a_{n}$ satisfying

$$
\sum_{1 \leq i<j \leq n} m_{j i}\left(e_{i}-e_{j}\right)+\sum_{i=1}^{n} a_{i}\left(e_{i}-e_{n+1}\right)=\left(1,2, \ldots, n,-\left(\begin{array}{c}
n+1 \\
2
\end{array}\right)\right) .
$$

This sets up a bijection between (a) and (b). This result is due to A. Postnikov and R. Stanley (unpublished), and is also discussed by W. Baldoni-Silva and M. Vergne, ibid. (§8).

6.C13 Let the vertices be $1,2, \ldots, 4 m+2$ in clockwise order. Suppose that there is a chord between vertex 1 and vertex $2 i$.

Case 1: $i$ is odd. The polygon is divided into two polygons, one (say $P_{1}$ ) with vertices $2, \ldots, 2 i-1$ and the other (say $P_{2}$ ) with vertices $2 i+1, \ldots, 4 m+2$. Choose $i-1$ noncrossing chords on the vertices $2,3, \ldots, 2 i-1$. Thus we have a net $N_{1}$ on $P_{1}$. Choose a coloring of the faces of $N_{1}$. By "symmetry" half the nets on $P_{2}$ will have an even number of blue faces and half an odd number (with respect to the coloring of the faces of $\left.N_{1}\right)$, so the number of nets with a chord $(1,2 i)$ and an even number of blue faces minus the number of nets with a chord $(1,2 i)$ and an odd number of blue faces is 0. (It's easy to make this argument completely precise.)

Case 2: $i$ is even, say $i=2 j$. The number of nets $N_{1}$ on $P_{1}$ with an even number of blue faces is $f_{e}(j-1)$. The number with an odd number is $f_{o}(j-1)$. Similarly the number of nets $N_{2}$ on $P_{2}$ with an even number of blue faces is $f_{e}(m-j)$ and with an odd number of blue faces is $f_{o}(m-j)$. Hence the number of nets on the original $4 m+2$ points with a chord $(1,4 j)$ and an even number of blue faces is $f_{e}(j-1) f_{e}(m-j)+f_{o}(j-1) f_{o}(m-j)$. Thus

$$
f_{e}(m)=\sum_{j=1}^{m}\left(f_{e}(j-1) f_{e}(m-j)+f_{o}(j-1) f_{o}(m-j)\right) .
$$

Similarly

$$
f_{o}(m)=\sum_{j=1}^{m}\left(f_{e}(j-1) f_{o}(m-j)+f_{o}(j-1) f_{e}(m-j)\right) .
$$


Hence

$$
f_{e}(m)-f_{o}(m)=\sum_{j=1}^{m}\left(f_{e}(j-1)-f_{o}(j-1)\right)\left(f_{e}(m-j)-f_{o}(m-j)\right),
$$

which is just the recurrence satisfied by Catalan numbers $C_{m}$ (initial condition $C_{0}=1$ ) and thus also by $D_{m}=(-1)^{m+1} C_{m}$ (initial condition $\left.D_{0}=-1\right)$. Since $f_{e}(0)-f_{o}(0)=0-1=-1$, the proof follows.

This result was first proved using techniques from algebraic geometry by A. Eremenko and A. Gabrielov, in Computational Methods and Function Theory, vol. 1, 2001, pp. 1-25. A bijective proof was given by S.-P. Eu, Coloring the net, preprint, based on the paper S.-P. Eu, S.-C. Liu, and Y.-N. Yeh, Discrete Math. 281 (2004), 189-196.

6.C14 This result is due to Gregory Berkolaiko, Jonathan Harrison, and Marcel Novaes, cond-mat/0703803. The result was originally suggested to Marcel Novaes by some calculations in random matrix theory. Subsequently Novaes and his collaborators found a generating function proof.

6.C15 This conjecture (stated differently) is due to J. Körner, C. Malvenuto, and G. Simonyi, SIAM J. Discrete Math. 22(2) (2008), 489-499 (§3.2). These authors show by a construction that $f(n) \geq C_{n}$. Update. The conjecture is false. Jineon Baek gave the following counterexample for $n=5$ (private communication 9 August 2012): $\{(1,2,4,8,16)$, $(2,3,4,8,16),(1,4,5,8,16),(4,5,6,8,16),(4,6,7,8,16),(1,2,8,9,16),(2,3,8,9,16)$, $(1,8,9,10,16),(1,8,10,11,16),(8,9,10,12,16),(8,10,11,12,16),(8,9,12,13,16)$, $(8,12,13,14,16),(8,12,14,15,16),(1,2,4,16,17),(2,3,4,16,17),(1,4,5,16,17)$, $(4,5,6,16,17),(4,6,7,16,17),(1,2,16,17,18),(2,3,16,20,21),(3,4,16,18,19)$, $(4,5,16,18,20),(2,4,16,19,20),(1,16,17,18,20),(1,16,18,19,20),(1,16,17,20,21)$, $(1,16,20,21,22),(1,16,20,22,23),(16,17,18,20,24),(16,18,19,20,24),(16,17,20,21,24)$, $(16,20,21,22,24),(16,20,22,23,24),(16,17,18,24,25),(16,18,19,24,25),(16,17,24,25,26)$, $(16,17,24,26,27),(16,24,25,26,28),(16,24,26,27,28),(16,24,25,28,29),(16,24,28,29,30)$, $(16,24,28,30,31)\}$. This set has 43 elements.

6.C16 (a) The techniques of R. Stanley, J. Combinatorial Theory, Ser. A 114 (2007), 436-460, can be used to show that the coefficients in question count 321-avoiding alternating permutations in $\mathfrak{S}_{2 m-1}$. Now use $\left(n^{7}\right)$. 
(b) Similarly to (a) we are counting 321-avoiding alternating permutations in $\mathfrak{S}_{2 m}$. Again use $\left(\mathrm{n}^{7}\right)$.

(c) Here we are counting 321-avoiding reverse alternating permutations in $\mathfrak{S}_{2 m}$. Now use $\left(\mathrm{o}^{7}\right)$.

6.C17 By polynomial interpolation it suffices to prove the two identities when $q$ is a positive integer.

(a) Let $G(x)=x C(x)$. Then $G(x)=\left(x-x^{2}\right)^{\langle-1\rangle}$, and the proof follows easily from the Lagrange inversion formula (Theorem 5.4.2). Alternatively, $C(x)^{q}$ (when $q \in \mathbb{P}$ ) is the generating function for plane binary forests with $q$ components. Now use Theorem 5.3.10 in the case $n=2 k+q, r_{0}=k+q, r_{2}=k$ (and all other $r_{i}=0$ ).

(b) This can be obtained from (a) by differentiating $(x C(x))^{q}$ with respect to $x$, or alternatively from the identity

$$
x C(x)^{q}=\frac{C(x)^{q-1}}{2}-\frac{(1-4 x) C(x)^{q-1}}{2 \sqrt{1-4 x}} .
$$

6.C18 (a) It is straightforward to verify that $E(x)^{2}=C\left(4 x^{2}\right)$, which is equivalent to (9). This result is due to L. Shapiro, private communication dated 24 May 2002.

6.C19 (a) It is easy to see that if $F(t)$ exists, then it is unique. Now it follows from $x C(t)^{2}-C(t)+1=0$ that

$$
\frac{1-x+x C(t)}{1-x+x^{2} t}=\frac{1}{1-x t C(t)} .
$$

Hence $F(t)=C(t)$.

(b) We have

$$
\frac{1}{1-x t C(t)}=\sum_{k \geq 0} x^{k} t^{k} C(t)^{k} .
$$

Hence by (a) and Exercise 6.C17(a) there follows

$$
\begin{aligned}
{\left[x^{k}\right] f_{n}(x) } & =\left[t^{n}\right] t^{k} C(t)^{k} \\
& =\left[t^{n-k}\right] C(t)^{k} \\
& =\frac{k}{n}\left(\begin{array}{c}
2 n-k-1 \\
n-k
\end{array}\right)=\frac{k}{n}\left(\begin{array}{c}
2 n-k-1 \\
n-1
\end{array}\right) .
\end{aligned}
$$


6.C20 This result is due to V. Liskovets and R. Pöschel, Discrete Math. 214 (2000), 173-191. For the case $p=2$, see I. Kovács, Sém. Lotharingien de Combinatoire (electronic) 51 (2005), Article B51h.

6.C21 See F. Ardila, J. Combinatorial Theory, Ser. A 104 (2003), 49-62, and J. E. Bonin, A. de Mier, and M. Noy, J. Combinatorial Theory, Ser. A 104 (2003), 63-94..

6.C22 (a) Answer: $f(x)=\sqrt{4-x^{2}} / 2 \pi$ for $-2 \leq x \leq 2$, and $f(x)=0$ for $|x| \geq 2$. This result is the basis of Wigner's famous "semicircle law" for the distribution of eigenvalues of certain classes of random real symmetric matrices (Ann. Math. 62 (1955), 548-569, and 67 (1958), 325-327). Wigner did not rigorously prove the uniqueness of $f(x)$, but this uniqueness is actually a consequence of earlier work of F. Hausdorff, Math. Z. 16 (1923), 220-248.

(b) Answer: $f(x)=\frac{1}{2 \pi} \sqrt{\frac{4-x}{x}}$ for $0 \leq x \leq 4$, and $f(x)=0$ otherwise, an easy consequence of (a).

6.C23 This is a result of S. Yakoubov, to appear.

6.C24 Let $w$ be the vertex of $F$ adjacent to $v$. Let $G$ denote $F$ with $v$ removed, and let $g(n)$ be the number of closed walks in $G$ of length $2 n$ beginning at $w$. Write $A(x)=\sum_{n \geq 0} f(n) x^{n}$ and $B(x)=\sum_{n \geq 0} g(n) x^{n}$. The definitions of $F$ and $G$ yield

$$
\begin{aligned}
& A(x)=1+x A(x) B(x) \\
& B(x)=1+x B(x)^{2}+x A(x) B(x) .
\end{aligned}
$$

Eliminating $B(x)$ gives $A(x)=1+x A(x)^{3}$, the equation satisfied by the generating function for ternary trees by number of vertices. This result first appeared in D. Bisch and V. Jones, Invent. math. 128 (1997), 89-157, and D. Bisch and V. Jones, in Geometry and Physics (Aarhus, 1995), Lecture Notes in Pure and Appl. Math., vol. 184, Dekker, New York, 1997. 


\section{CHRONOLOGY OF NEW PROBLEMS (beginning 12/17/01)}

A number in brackets is the number of items (combinatorial interpretations of $C_{n}$ ) in Exercise 6.19 up to that point.

6.19 $\left(\mathrm{m}^{4}\right)$ December 17, 2001

$\mathbf{6 . 1 9}\left(\mathrm{h}^{6}\right)$ December 17, 2001

6.38(n) December 17, 2001

6.C17 December 17, 2001

6.C19 December 17, 2001

$\mathbf{6 . 1 9}\left(\mathrm{p}^{5}\right)$ January 29, 2002

6.C9 January 29, 2002

6.19 $\left(\mathrm{n}^{5}\right)$ March 20, $2002[90]$

6.19(ppp) April 29, 2002

6.19 $\left(\mathrm{j}^{4}\right)$ April 30, 2002

6.19 $\left(\mathrm{k}^{4}\right)$ April 30, 2002

6.19 $\left(\mathrm{f}^{8}\right)$ May 31, 2002

6.19(qqq) June 1, 2002

6.C18 June 1, 2002

6.19 $\left(\mathrm{w}^{7}\right)$ June 2, 2002

6.19 $\left(\mathrm{o}^{6}\right)$ June 4, 2002

6.C3 June 8, 2002

6.19 $\left(\mathrm{b}^{8}\right)$ July 1, 2002

6.19$\left(1^{6}\right)$ August 9, 2002 
6.C5 August 9, 2002

6.19(i $\left(\mathrm{i}^{4}\right)$ October 23, $2002[100]$

6.19 $\left(\mathrm{k}^{8}\right)$ October 23, 2002

6.19 $\left(\mathrm{s}^{5}\right)$ October 27, 2002

6.25(l) December 20, 2002

6.19 $\left(\mathrm{k}^{6}\right)$ March 12, 2003

6.19 $\left(\mathrm{v}^{6}\right)$ March 12, 2003

6.19 $\left(\mathrm{o}^{5}\right)$ April 6, 2003

6.19 $\left(\mathrm{m}^{7}\right)$ April 6, 2003

6.C2(b) (updated) April 17, 2003

6.19 $\left(\mathrm{n}^{7}\right)$ June 4, 2003

6.C4 October 30, 2003

6.C24 November 11, 2003

6.19 $\left(\mathrm{m}^{5}\right)$ April 20, 2004

6.19 $\left(\mathrm{r}^{6}\right)$ May 12, 2004 (solution modified 5/16/04)

6.19 $\left(\mathrm{x}^{7}\right)$ June 1, $2004[110]$

6.19 $\left(\mathrm{y}^{7}\right)$ June 2, 2004

6.C2(d) (updated) June 3, 2004

6.C22(b) June 3, 2004

6.19 $\left(\mathrm{z}^{7}\right)$ June 28, 2004

6.19 $\left(\mathrm{f}^{6}\right)$ August 22, 2004

6.19( $\left(\mathrm{e}^{8}\right)$ August 22, 2004

6.19(i $\left.{ }^{5}\right)$ August 22, 2004 
6.19 $\left(\mathrm{h}^{8}\right)$ September 2, 2004

6.19 $\left(\mathrm{v}^{8}\right)$ September 2, 2004

6.19 $\left(q^{4}\right)$ September 20, 2004

6.19 $\left(\mathrm{e}^{5}\right)$ November 13, 2004

6.C11 November 24, 2004

6.19 $\left(\mathrm{n}^{8}\right)$ November 25, 2004 [120]

6.25(m) December 17, 2004

6.19(i $\left.{ }^{6}\right)$ December 18, 2004

6.19 $\left(b^{4}\right)$ December 18, 2004

6.19 $\left(\mathrm{f}^{5}\right)$ December 20, 2004

6.19 $\left(\mathrm{h}^{5}\right)$ December 22, 2004

6.19(i $\left.{ }^{7}\right)$ January 16, 2005

$\mathbf{6 . 1 9}\left(\mathrm{j}^{7}\right)$ January 16, 2005

6.19 $\left(\mathrm{p}^{6}\right)$ February 11, 2005

6.19 $\left(q^{5}\right)$ February 28, $2005\left[2^{7}\right]$

6.19$\left(\mathrm{r}^{5}\right)$ April 8, 2005

6.19 $\left(\mathrm{n}^{6}\right)$ May 27, 2005 [130]

6.C20 September 7, 2005

6.19 $\left(\mathrm{s}^{4}\right)$ September 9, 2005

6.19 $\left(\mathrm{t}^{4}\right)$ September 9, $2005\left[132=C_{5}\right]$

6.19 $\left(u^{4}\right)$ September 9, 2005

6.39(t,u) October 14, 2005

6.19 $\left(\mathrm{t}^{5}\right)$ October 14, 2005 
6.C9(c) (revised) October 14, 2005

6.19(ttt) October 26, 2005

6.19 $\left(\mathrm{c}^{5}\right)$ April 24, 2006

6.19 $\left(\mathrm{w}^{6}\right)$ June 19, 2006

6.19 $\left(q^{7}\right)$ August 9, 2006

6.19 $\left(\mathrm{r}^{7}\right)$ August 9, 2006

6.19 $\left(d^{4}\right)$ August 11, 2006 [140]

6.19(h $\left.\mathrm{h}^{4}\right)$ August 11, 2006

6.39(v) August 16, 2006

6.19 $\left(\mathrm{x}^{6}\right)$ December 20, 2006

6.C14 December 22, 2006

6.19 $\left(\mathrm{y}^{6}\right)$ December 23, 2006

6.19 $\left(\mathrm{v}^{4}\right)$ February 11, 2007

6.19 $\left(\mathrm{w}^{4}\right)$ February 11, 2007

$\mathbf{6 . 1 9}\left(\mathrm{x}^{4}\right)$ February 11, 2007

6.19(yyy) February 23, 2007

6.19 $\left(\mathrm{y}^{4}\right)$ May 13, 2007

6.38(o) May 14, 2007

6.19 $\left(\mathrm{e}^{6}\right)$ June 20, 2007

6.19(zzz) September 2, 2007 [150]

6.19 $\left(\mathrm{e}^{4}\right)$ September 2, 2007

6.19 $\left(\mathrm{z}^{6}\right)$ September 2, 2007

6.19 $\left(c^{7}\right)$ September 2, 2007 
6.25(n) September 3, 2007

6.19 $\left(1^{7}\right)$ September 9, 2007

6.19 $\left(1^{4}\right)$ September 13, 2007

6.19 $\left(\mathrm{k}^{5}\right)$ September 14, 2007

6.19 $\left(\mathrm{o}^{4}\right)$ September 15, 2007

6.19 $\left(1^{5}\right)$ September 15, 2007

6.19 $\left(\mathrm{p}^{4}\right)$ September 16, 2007

6.19(rrr) September 16, 2007 [160]

6.19 $\left(g^{4}\right)$ September 16, 2007

6.C3(c) November 27, 2007

6.19(uuu) February 22, 2008

6.19 $\left(q^{6}\right)$ February 22, 2008

6.19 $\left(\mathrm{a}^{8}\right)$ February 22, 2008

6.25(o) March 28, 2008

6.19( $\left.\mathrm{a}^{7}\right)$ April 26, 2008

6.25(p) May 15, 2008

6.19(sss) May 15, 2008

6.C7 July 18, 2008

6.C15 October 5, 2008

6.19 $\left(\mathrm{m}^{8}\right)$ February 24, 2009

6.19 $\left(\mathrm{s}^{8}\right)$ February 24, 2009

6.19 $\left(u^{8}\right)$ April 2, 2009

6.39(w) April 3, 2009 
6.19(www) April 29, 2009 [170]

$\mathbf{6 . 1 9}\left(\mathrm{y}^{5}\right)$ June 10, 2009

6.19 $\left(\mathrm{r}^{8}\right)$ June 10, 2009

6.19 $\left(\mathrm{o}^{7}\right)$ August 9, 2009

6.C16 August 11, 2009

6.38(p) August 26, 2009

6.19 $\left(\mathrm{d}^{8}\right)$ December 27, 2009

6.19 $\left(\mathrm{p}^{7}\right)$ December 27, 2009

6.19 $\left(0^{8}\right)$ December 27, 2009

6.19 $\left(\mathrm{w}^{8}\right)$ April 27, 2010

6.19 $\left(\mathrm{b}^{6}\right)$ April 27, 2010

6.19 $\left(1^{8}\right)$ May 5, 2010

6.19 $\left(\mathrm{b}^{7}\right)$ June 1, 2010 [180]

6.19 $\left(\mathrm{d}^{7}\right)$ June 3, 2010

6.19(vvv) June 4, 2010

6.19 $\left(\mathrm{j}^{5}\right)$ June 4, 2010

6.19(e $\left.\mathrm{e}^{7}\right)$ June 5, 2010

6.19 $\left(\mathrm{f}^{7}\right)$ June 5, 2010

6.19 $\left(g^{7}\right)$ June 5, 2010

6.19 $\left(\mathrm{h}^{7}\right)$ June 5, 2010

6.19( $\left.c^{8}\right)$ July 5, 2010

6.19( $\left.\mathrm{k}^{7}\right)$ August 21, 2010

6.C8 February 18, 2011 

6.19 $\left(\mathrm{v}^{5}\right)$ April 30, $2010[190]$
6.19 $\left(\mathrm{w}^{5}\right)$ April 30, 2010
6.19 $\left(\mathrm{m}^{6}\right)$ April 30, 2010
6.19 $\left(c^{6}\right)$ October 22,2011
6.19 $\left(\mathrm{t}^{6}\right)$ October 22, 2011
6.19 $\left(\mathrm{u}^{6}\right)$ October 22, 2011
$\mathbf{6 . 1 9}\left(\mathrm{q}^{8}\right)$ October 22,2011
6.19 $\left(\mathrm{x}^{5}\right)$ October 22, 2011
6.19 $\left(\mathrm{u}^{5}\right)$ October 23, 2011

June 15, 2012. An item has been deleted. $\ddot{6}$ This is the 321-avoiding derangements of $[n+1]$. The number for $n=3$ is six, not five.

6.19( $\left(\mathrm{a}^{5}\right)$ July 12, 2012

6.19$\left(b^{5}\right)$ July 12, 2012

$\mathbf{6 . 1 9}\left(\mathrm{g}^{8}\right)$ July 13, 2012 [200]

6.C15 (updated) August 12, 2012

6.C6 April 1, 2013

6.19 $\left(\mathrm{r}^{4}\right)$ April 10, 2013

6.39(x) April 14, 2013

6.19(xxx) April 22, 2013

6.19 $\left(\mathrm{y}^{8}\right)$ May 2, 2013

6.C23 May 2, 2013

6.19 $\left(\mathrm{p}^{8}\right)$ May 15, 2013

6.19( $\left(\mathrm{a}^{6}\right)$ May 24, 2013 
6.C1 May 25, 2013

6.C21 May 25, 2013 